\title{
ANALYSIS OF PRICE TRANSMISSION AND ASYMMETRIC ADJUSTMENTS USING BAYESIAN ECONOMETRIC METHODOLOGY
}

\author{
Dissertation \\ Submitted for the Degree of Doctor of Philosophy \\ at the Faculty of Agricultural Sciences \\ Georg-August-University of Göttingen, Germany \\ by \\ Henry De-Graft Acquah \\ born in Takoradi, Ghana
}

Göttingen, January 2008 
D7

$1^{\text {st }}$ examiner: Professor Dr. Stephan von Cramon-Taubadel $2^{\text {nd }}$ examiner: Professor Dr. Steffen Kühnel

Date of oral examination: $31^{\text {st }}$ January 2008 


\section{Dedication}

To my parents;

Ama Nkansah and Nana Kwao Kofie III

And with many thanks to the Most High God 


\begin{abstract}
Within the econometric models of asymmetric price transmission, different specifications which detect asymmetry at different rates or culminate in different inferences and conclusions have been developed. However, the goal of asymmetric price transmission modelling is to select a single model from a set of competing models that best captures the underlying asymmetric data generating process for derivation of policy conclusions. This leads to issues of model comparison and model selection, measuring the relative merits of alternative specifications and using the appropriate criteria to choose the most reliable method or model specification which best fits or explains a given set of data.
\end{abstract}

The Bayesian theory which provides a flexible and conceptually simple framework for comparing competing models is theoretically introduced and demonstrated in the price transmission models. On the basis of Marginal Likelihood and Information-theoretic Selection Criteria, alternative methods of testing for asymmetry are evaluated when the true asymmetric data generating process is known. Using a Monte Carlo simulation of model selection, the performance of a range of model selection algorithms to clearly identify the true asymmetric data generating process is examined and the effects of the amount of noise in the model, the sample size and the difference in the asymmetric adjustment parameters on model selection are also simulated.

The results of 1000 Monte Carlo simulation indicates that information criteria and the marginal likelihood provides a holistic and consistent approach to ranking and selecting among the competing models of asymmetric price transmission. Estimation results with all simulated data are accurate for the true model and the marginal likelihood and information criterion clearly identifies the correct model out of alternative competing models or on the average points to the true asymmetric data generating process. The Monte Carlo simulation results further indicates that the sample size, the difference in the asymmetric adjustment parameters, the number of asymmetric adjustment parameters (i.e. model complexity) and the amount of noise in the model are important in identifying the true asymmetric data generating process. Subsequently, the ability of the model selection procedures to recover the true asymmetry data generating process(i.e. Model Recovery Rates) increases with increases in the difference between the asymmetric adjustments parameters, increases in sample size, 
increases in number of asymmetric adjustment parameters (i.e. complexity of the true model) and decreases in the amount of noise in the model. Intuitively, the number of informative variables used to model an asymmetry may improve the recovery of the true data generating process. Importantly, model selection may have difficulty in identifying the true asymmetric model at higher noise levels or performance of the model selection methods in recovering the true model deteriorates at higher noise levels in the asymmetric price transmission modeling framework. Generally, larger sample sizes may improve the ability of the Bayesian criteria to make correct inferences about the asymmetric price transmission models. As expected, model fit declined with increases in stochastic variance in the asymmetric price transmission models analysed. Similarities exist between the performance of the marginal likelihood and its approximations (BIC) and (DIC). The marginal likelihood gives the same model ranking when compared with the Bayesian Information Criteria (BIC), suggesting that the BIC could be used as a complementary approach. The Monte Carlo simulation results indicate that a relatively new information criterion, Drapers's Information Criteria (DIC), which shares the features of the Bayesian Information Criteria, performs similarly to or better than the BIC in the price transmission modeling framework on the basis of the recovery rates of the true asymmetric data generating process. Importantly, the factors that affect the performance of the model selection methods in recovering the true asymmetric data generating process are also influential in the power test of asymmetry.

Methodologically, the comparison provided contributes to knowledge and understanding of the empirical performance of the marginal likelihood and information criteria (i.e. Model Selection Methods) in an asymmetric price transmission modeling framework for which no studies have been undertaken. Researchers can apply the Bayesian criteria, knowing from this research that the Bayesian criteria on average do points to the true data generating process in the asymmetric price transmission modeling framework. The results of various Monte Carlo experiments reinforce the importance of design informativeness in an asymmetric price transmission modeling framework and suggest the conditions under which the ability of the model selection methods in identifying the true asymmetric model that governs a given data will improve. Similarly, the conditions which will improve the power of the test for asymmetry are also suggested. The model recovery simulations exemplified will serve as a useful tool for investigating model selection problems in other applications. 


\section{Acknowledgements}

For their contribution to the dissertation process, I express my appreciation to the following: For his wisdom, insight, guidance, patience and generosity, I am deeply indebted to my advisor Prof. Dr. Stephan von Cramon-Taubadel. Thanks to Prof. Dr. Steffen Kühnel, Prof. Dr. Walter Zucchini and Prof. Dr. Bernhard Brümmer for accepting to be my second, third and fourth examiners respectively. Prof. Dr. Stephan von Cramon-Taubadel has my gratitude for providing financial assistance for the Ph.D studies.

Throughout the Ph.D work I have experienced the support of many. I wish to thank Prof. Dr. Manfred Denker and the faculty of the Centre of Statistics, University of Göttingen for their efforts on my behalf. Many thanks to Prof. Dr. Gary Koop of the University of Stracthclyde and Prof. Dr. John Geweke of the University of Iowa for comments and suggestions they provided during the initial stages of this work. Special thanks to Dr. Oleg Nenadic of the Institute of Statistics and Econometrics, University of Göttingen for numerous help he provided in statistical programming. I would also like to thank my colleagues at the Department of Agricultural Economics and Rural Development and in particular Antje Wagener for all the support.

I was fortunate to experience the friendship and support of many people (some anonymous) including: Isaac Abunyuwah, Patrick Arthur, James Arthur, Axel Buschmann, Amos Gyau, Edward Onumah, Asiamah Bruku, Atta Ofori and Dinah Ofori. In particular, I would like to thank Axel Buschmann for his constant support throughout my graduate studies.

Special thanks go to the entire Acquah family for the encouragement and prayer support that has seen this study to a successful completion.

Finally to the Most High God be all glory for granting exceedingly and abundantly above what I can think or imagine. 
Table of Contents

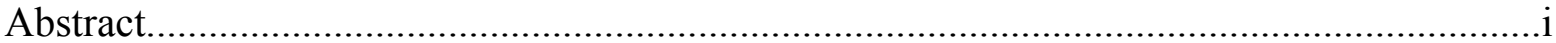

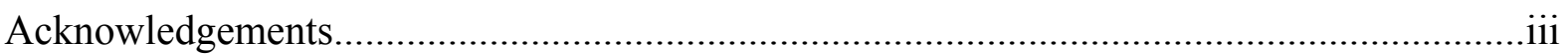

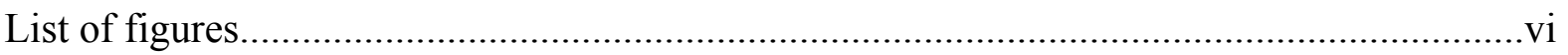

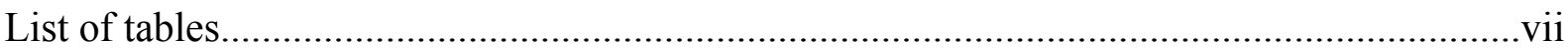

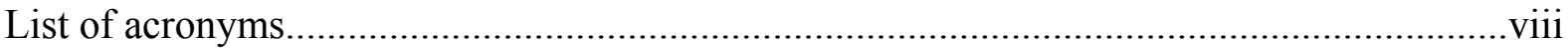

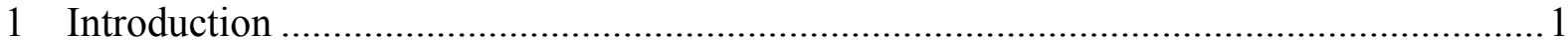

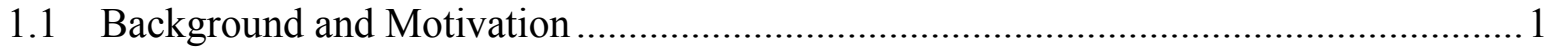

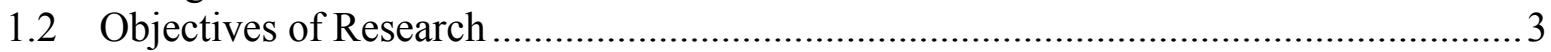

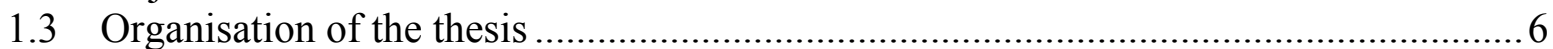

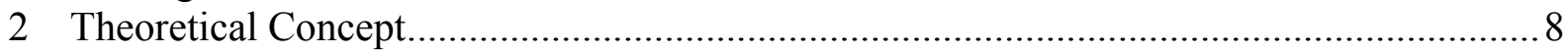

2.1 Asymmetric Price Transmission.......................................................................... 8

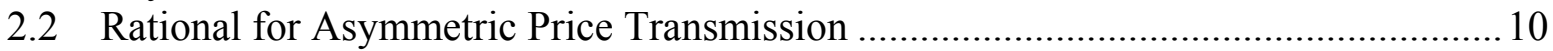

2.3 Econometric Modeling of Asymmetric Price Transmission ...................................... 12

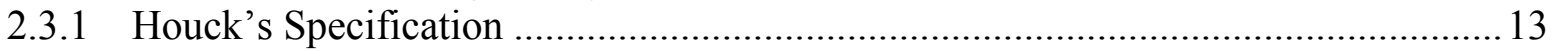

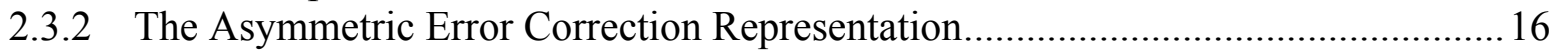

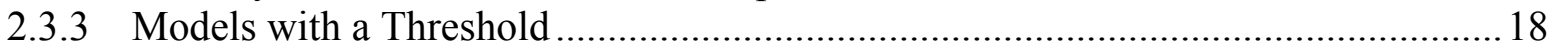

2.4 Empirical Overview of the Different Asymmetry Methods...................................... 19

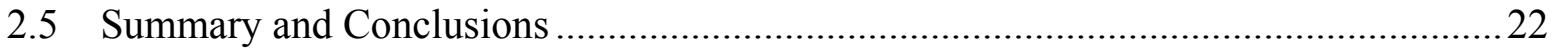

3 Analysing Asymmetric Price Transmission using Bayesian Econometric Methodology.. 24

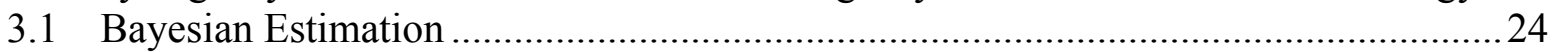

3.2 Bayesian Model Comparison and Choice ................................................................. 27

3.3 Implementing Bayesian Model Choice in Practice ..................................................28

3.4 Bayesian Analysis of the Standard Regression Model ..............................................28

3.4.1 The Regression Model of Asymmetric Price Transmission Analysis ........................29

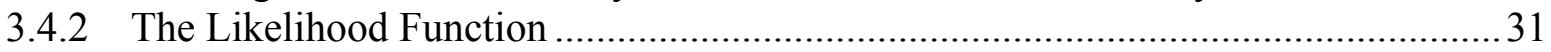

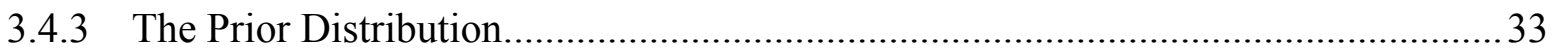

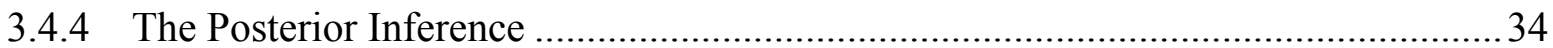

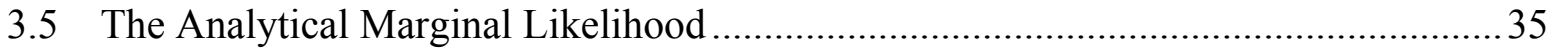

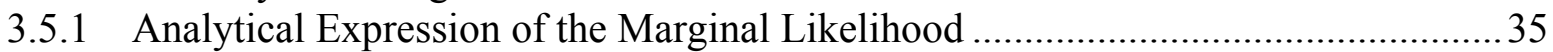

3.5.1.1 Derivation of the Marginal Likelihood Using Normal Gamma Prior ................. 35

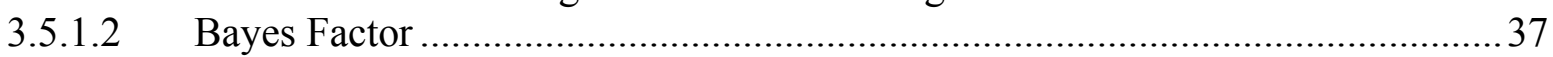

3.5.1.3 Prior Sensitivity Analysis .......................................................................... 38

3.5.1.4 Derivation of the Marginal Likelihood Using No Prior Knowledge................... 38

3.5.2 Marginal likelihood Approximation Using Bayesian Information Criteria (BIC) .... 40

3.5.3 Marginal likelihood Approximation Using Draper's Information Criteria (DIC) .... 41

3.6 An Overview of the Bayesian Model Selection Criteria ........................................... 41

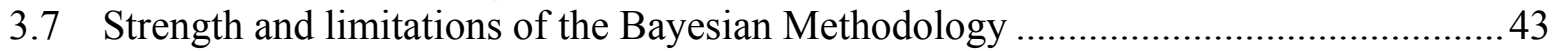

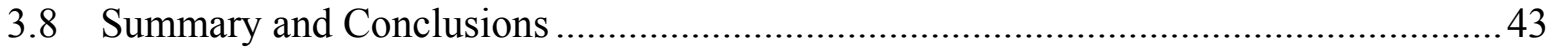

4 A Monte Carlo Comparison of Marginal Likelihoods and Information Criteria in the Econometric Models of Asymmetric Price Transmission ................................................. 46

4.1 Data Simulation Experiment ............................................................................ 46

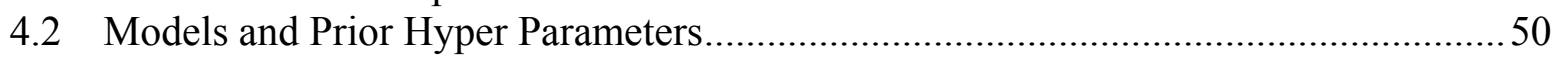

4.3 Bayesian Model Assessment using the Marginal likelihood........................................ 51

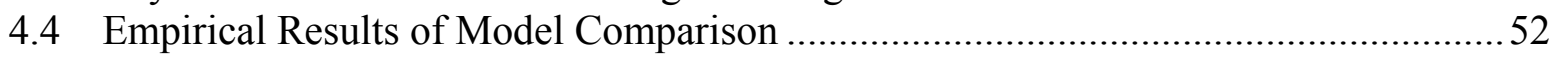

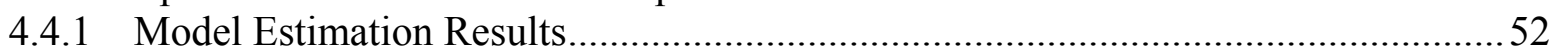


4.4.2 Evaluation of Model Performance using Rankings derived from the Analytical Marginal Likelihood with a Natural Conjugate Prior.............................................56

4.5 Prior Sensitivity Analysis of the Analytical Marginal Likelihood ...............................60

4.6 Analysing the Model Recovery Rates of the Tests for Asymmetry .............................62

4.6.1 Monte Carlo Simulation of the Effects of Sample Size on Model Selection ............63

4.6.2 Monte Carlo Simulation of the Effects of Stochastic Variance on Model Selection 66

4.6.3 Monte Carlo Simulation of the Effects of Difference in Asymmetric Adjustment Parameters on Model Selection .......................................................................... 70

4.7 Overview of the Performance Analysis of the Different Asymmetry Test using Simulated Data generated from the Complex Asymmetric ECM …...........................72

4.7.1 Empirical Comparison of Model Selection Criteria ................................................. 75

4.7.2 Monte Carlo Simulation of the Effects of Sample Size on Model Recovery............76

4.7.3 Monte Carlo Simulation of the Effects of Noise levels on Model Recovery ............ 78

4.7.4 Monte Carlo Simulation of the Effects of Difference in Asymmetric Adjustment Parameters on Model Selection (CECM) ................................................................... 83

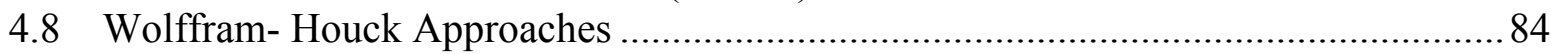

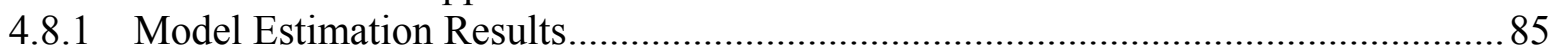

4.8.2 Evaluation of Model Performance using Rankings derived from the Analytical Marginal Likelihood with a Natural Conjugate Prior (SWM) ............................... 87

4.8.3 Sensitivity of the Analytical Marginal Likelihood to the Prior ................................. 91

4.8.4 Monte Carlo Simulation of the Effects of Sample Size on Model Selection ............ 92

4.8.5 Monte Carlo Simulation of the Effects of Stochastic Variance on Model Selection 96

4.8.6 Monte Carlo Simulation of the Effects of Difference in Asymmetric Adjustment

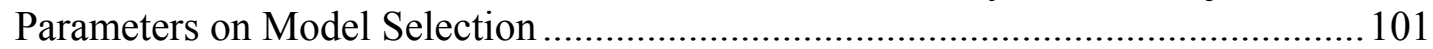

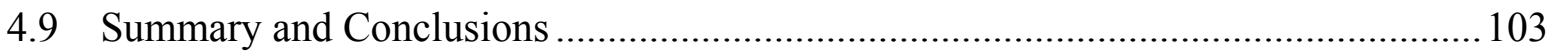

5 An Empirical Comparison of the Power of Tests for Non-Linearity in the Econometric

Models of Asymmetric Price Transmission ....................................................................... 107

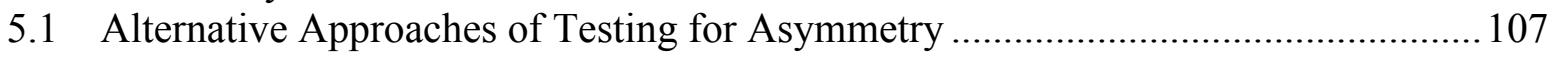

5.2 A Monte Carlo Analysis of the Power of tests for Asymmetry................................. 108

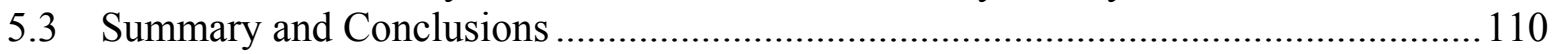

6 Conclusion, Implications and Directions for Future Research..................................... 112

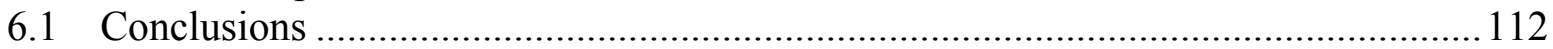

6.2 Methodological Implications for Asymmetric Price Transmission Modeling ........... 117

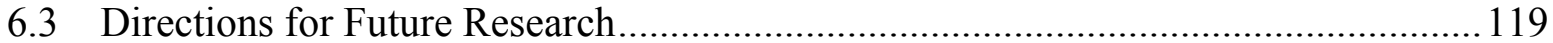

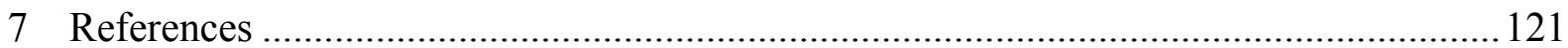

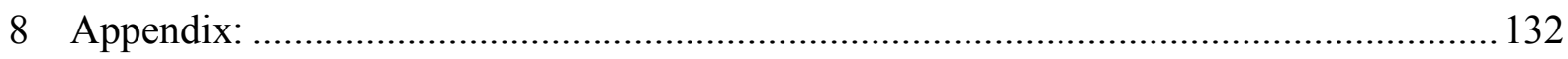




\section{List of figures}

Figure 1: Types of Asymmetry (Speed and Magnitude) .............................................. 10

Figure 2: Bayesian Framework for model selection.................................................... 42

Figure 3: Effects of Sample Size on Model Selection.................................................... 65

Figure 4: Effects of Stochastic Variance on Model Selection......................................... 68

Figure 5: Effects of Stochastic Variance and Sample Size on Model Selection.............. 69

Figure 6: Example of Model fit as a function of Error Size........................................ 70

Figure 7: Example of the Effect of the level of Asymmetry....................................... 71

Figure 8: Effects of Sample Size on Model Recovery.................................................... 77

Figure 9: Effects of Noise level on Model Recovery.................................................... 80

Figure 10: Concurrent Effects of Sample Size and Noise level on Model Recovery ..... $\quad 81$

Figure 11: Model fit as a function of Noise levels.................................................... 83

Figure 12: Example of the Effect of the level of Asymmetry...................................... 84

Figure 13: Effects of Sample Size on Model Selection............................................. 94

Figure 14: Effects of Stochastic Variance on Model Selection...................................... 98

Figure 15: Effects of Stochastic Variance and Sample Size on Model Selection........... 99

Figure 16: Model fit as a function of Noise levels (SWM)......................................... 101

Figure 17: Example of the Effect of the level of Asymmetry..................................... 102 
List of tables

Table 1: Asymmetry Test of the Different Econometric Models.................................... 20

Table 2: Standard ECM DGP (1): Natural Conjugate Prior, Least Squares and 54 Posterior Moments of the SECM (Analytical Method)....

Table 3: Standard ECM DGP (2): Natural Conjugate Prior, Least Squares and Posterior Moments of the SECM (Analytical Method).

Table 4: Standard ECM DGP (1): Analytical Marginal Likelihood (BMS), BIC, DIC and AIC of selected models

Table 5: Standard ECM DGP (2): Analytical Marginal Likelihood (BMS), BIC, DIC and AIC of Selected models.

Table 6: Standard ECM DGP (1): Sensitivity of the Analytical Marginal Likelihood to the Prior (SECM)....

Table 7: Standard ECM DGP (2): Sensitivity of the Analytical Marginal Likelihood to the Prior (SECM).

Table 8: Relative Performance of Model Selection Methods across Sample Size

Table 9: Relative Performance of Model Selection Methods across Noise Levels

Table10: Complex ECM DGP: Analytical Marginal Likelihood (BMS), BIC, DIC and AIC of selected models (CECM).

Table11: Sensitivity of the Analytical Marginal Likelihood to the Prior (CECM)

Table 12: Natural Conjugate Prior, Least squares and Posterior Moments of the CECM (Analytical Method)

Table13: Effects of Sample Size on Model Recovery

Table14: Effect of Stochastic Variance on Model Recovery

Table15: Wolffram DGP (1): Natural Conjugate Prior, Least Squares and Posterior Moments of the SWM (Analytical Method).

Table16: Wolffram DGP (2): Natural Conjugate Prior, Least Squares and Posterior Moments of the SWM (Analytical Method).

Table 17: Wolffram DGP (1): Analytical Marginal Likelihood (BMS), BIC, DIC and AIC of selected models.

Table 18: Simple Wolffram DGP (2): Analytical Marginal Likelihood (BMS), BIC, DIC and AIC of selected models.

Table 19: Wolffram DGP (1): Sensitivity of the Analytical Marginal Likelihood to the Prior (SWM).

Table 20: Wolffram DGP (2): Sensitivity of the Analytical Marginal Likelihood to the Prior (SWM).

Table 21: Relative Performance of Model Selection Methods across Sample Size .......... 93

Table 22: Relative Performance of Model Selection Methods across Noise Levels

Table 23: Rejection frequencies based on 10000 Replications. 109

Table 24: Rejection frequencies based on 10000 Replications 
List of acronyms

AIC Akaike's Information Criteria

BF Bayes Factor

BIC Bayesian Information Criteria

BMS Bayesian Model Selection Using the Marginal Likelihood

CECM Complex Asymmetric Error Correction Model

DGP Data Generating Process

DIC Draper's Information Criteria

ECT Error Correction Term

FAO Food and Agricultural Organization

HK Houck's Model

HKD Houck's Model in First Differences

HKS Houck's Model in Sum Differences

MCMC Markov Chain Monte Carlo algorithm

SECM Standard Asymmetric Error Correction Model

SWM Simple Wolffram Model

W-H Wolffram Houck Approaches 


\section{Introduction}

\subsection{Background and Motivation}

The analysis of price transmission and asymmetric adjustment has matured over the years with many developments in model specification, estimation and testing. Among these developments are the construction and application of various econometric models of asymmetric price transmission.

These models include an econometric model specification introduced by Wolffram (1971) and later refined by Houck (1977), the error correction model (von Cramon-Taubadel, 1994, 1998) and models with a threshold (Tsay, 1989; Balke and Fomby, 1997; Enders and Granger, 1998; Goodwin and Holt, 1999; Goodwin and Harper, 2000; Goodwin and Piggott, 2001; Abudulai, 2002; Cook and Holly, 2002; Goodwin and Serra, 2003; Cook, 2003). Different kinds of these models have been extensively used in analysing price transmission and testing for asymmetric adjustment, but there seems to be no specific accepted way of determining which is appropriate and under which setting.

Within the pre-cointegration setting, the variant of the Houck's model (HK) includes specification in first differences and recursive sum of first differences. In the post cointegration setting, variants of the Error Correction Models (ECM) specified includes the standard error correction representation (Granger and Lee, 1989; von Cramon-Taubadel, 1998) and an error correction model with complex dynamics (von Cramon-Taubadel and Loy, 1996). Additionally, variants of the threshold model have been specified by various authors (Godwin and Piggott, 2001; Abdulai, 2002; Hansen and Seo, 2002; Cook, 2003; Meyer, 2003).

Although the alternative methods or models are continually used in analysing price dynamics and testing for asymmetric adjustments to derive policy conclusions, they remain incompatible with one another and may result to differences in inference and conclusions. In this light, von Cramon-Taubadel and Loy (1999) extended the application of the asymmetric ECM and concluded that this method was more appropriate than the use of the conventional Houck approach if the data under investigation were cointegrated. Similarly, in support of the fact that different methods employed to detect asymmetric price transmission may lead to 
different conclusions, Capps and Sherwell (2007) find that the inference and conclusions derive from the von Cramon-Taubadel and Loy ECM approach was not supported by the conventional Houck approach in an empirical application.

Moreover, practitioners do not appear to use a common, objective set of criteria to choose the different methods that are employed in different settings. However, the lack of rigorous and common, objective set of model selection criteria ${ }^{1}$ is not satisfying since a survey of literature by Meyer and von Cramon-Taubadel (2004) also demonstrate that the alternative approaches detect asymmetry at different rates. In agreement with von Cramon-Taubadel et al. (2004) this research uses artificial data to demonstrate that different methods detect asymmetry at different rates (See appendix I).

Additionally, Frey and Manera $^{2}$ (2007) assert that no attempt has been made to address the issue of which of the various asymmetric price transmission models is most reliable or fits a given data set better, despite the numerous empirical research undertaken. In effect, there has been very little basis for choosing between the different methods. There is also the issue of different susceptibilities to data anomalies (i.e. structural break and their impact on the measures of asymmetry) detailed in von Cramon-Taubadel and Meyer (2000).

Thus, with many competing models in existence and a tendency to develop new models without accounting for the choice of statistical model, it is increasingly evident that the issue of selecting a plausible method from the different asymmetry test using a model selection strategy remains an important methodological issue within the econometric models of asymmetric price transmission. Fundamentally, determining the true underlying asymmetric data generating process is imperative since the different methods may lead to different inferences and conclusions ${ }^{3}$. Wasserman (2000) affirms that in a model selection context where the true model is excluded from the set of competing models, the model that best approximates the true data generating process will be selected by the Bayesian approach.

This research fills the gap by addressing the methodological issues of model comparison and selection in the alternative econometric models of asymmetric price transmission. The

\footnotetext{
${ }^{1}$ It is rare for a published article to describe in detail the procedures actually followed in selecting models. Conventionally, researchers derive models to be estimated from theory and prior research.

${ }^{2}$ Detail discussions are provided in the Journal of Economic Surveys

${ }^{3}$ Capps and Sherwell (2007) details the discussion.
} 
author's contributions lie in theoretically introducing and developing the Bayesian analysis of the asymmetric price transmission models within the context of a conjugate analysis and demonstrating their application across alternative asymmetric price transmission methods or model specifications during model selection. This entails the provision or application of Bayesian algorithms as flexible model selection strategies to decide between the alternative approaches of testing for asymmetry. Empirically, the research evaluates and presents comparisons of the relative performance of the model selection algorithms in an asymmetry price transmission modeling framework.

In effect, the contribution of this research is not limited to introduction and application of the Bayesian methods but expands to incorporate the empirical comparison of the model selection methods in an asymmetric price transmission modeling framework for which no studies have been undertaken.

Specifically, the concept of the marginal likelihood and Information Criteria is introduced to the asymmetric price transmission modeling community and its implementation in a rigorous model comparison is illustrated with a theoretical and an empirical analyses. Emphasis is given to the development and adaptation of algorithms within a Bayesian framework to address the problem of model selection and demonstrate their viability with application to artificial data in asymmetric price transmission analysis. This involves using simulated asymmetric data generated from a specific model to determine whether the marginal likelihood and Information Criteria are capable of identifying the true model from which the data was generated (e.g. Asymmetric Error Correction Model Data Generating Process).

\subsection{Objectives of Research}

This research aims to introduce and demonstrate how Bayesian Methods can be used to address the methodological issues of model comparison and selection between the competing econometric models of asymmetric price transmission with an emphasis on the empirical comparison of the relative performance of model selection methods across the different asymmetry test. The thesis investigates the alternative methods of testing for asymmetry and 
determines whether the Bayesian model selection ${ }^{4}$ criteria are capable of identifying the true asymmetric price transmission model from which a simulated data is generated.

The measures of model fit such as the marginal likelihood and Information Criteria are introduced to asymmetric price transmission modeling community and their application in a rigorous model comparison and selection analysis via Monte Carlo experimentation is demonstrated.

Empirically, simulated data is generated from a specific model to illustrate the usefulness of the Bayesian Methods in comparing the asymmetric price transmission models. The performance of the Bayesian Methods to discriminate between different asymmetric price transmission models and the ability of the Bayesian Criteria to clearly identify the underlying asymmetry data generating process for the simulated data is examined via Monte Carlo Simulations.

The specific objectives of this research are to:

1) Develop and implement the Bayesian Analysis of the asymmetric price transmission regression model.

This entails introducing the Bayesian estimation and using a theoretical analysis to derive the prior distribution for the asymmetric price transmission regression specification. Essentially, a prior distribution which has the same functional form as the likelihood function is deduced (i.e. a Normal Gamma Prior distribution or Natural Conjugate Prior). The covariates of the regression model are used to construct the prior hyper parameters of the prior distribution in the spirit of Zellner (1986b). Extending the theoretical analysis, the data information is combined with the prior information to obtain the posterior distribution which forms the basis of all inference about unknown parameters in the model. This completes parameter estimation in the Bayesian analysis and forms a basis for model comparison and selection. With sample from the posterior distribution, almost any quantity of interest can be estimated. For example,

\footnotetext{
${ }^{4}$ Model selection refers to the problem of using the data to select one model from the list of candidate models. Essentially, it involves the use of a model selection criterion to find the best fitting model to the data (Wasserman, 2000).
} 
integrating across the posterior distribution with respect to the unknown parameters in the model yields the Marginal Likelihood.

2) Submit selected econometric models of asymmetric price transmission to a Bayesian model selection technique based on the concept of the marginal likelihood and information criteria.

a.) Evaluate the performance of the marginal likelihood and Information Criteria in identifying the true asymmetric data generating process.

This objective entails using simulated asymmetric data generated from a specific model (e.g. Asymmetric Error Correction Model data generating process) to determine whether the analytical marginal likelihood and associated measures are capable of identifying the true model out of the different alternative models. This involves measuring the relative merits of the different specifications and using appropriate criteria to choose the most probable or best fitting model given the simulated data. Rankings for the different models are derived using the marginal likelihood as a criterion. Additionally, different models are compared using approximation of the marginal likelihood. Subsequently, the performance of the Bayesian Information Criteria and Drapers Information Criteria is discussed in relation to the analytical marginal likelihood, taking into consideration the relevant assumptions. The performance of the Bayesian methods is compared with the commonly used Akaike Information Criteria. Fundamentally, the usefulness of the marginal likelihood and information criteria in model comparison and selection between the econometric models of asymmetric price transmission is demonstrated.

b.) Demonstrate the conditions which improve the ability of the marginal likelihood and information criteria to recover the true asymmetric data generating process in a Monte Carlo Experimentation.

Importantly, the performance of the Bayesian model selection technique is evaluated using Monte Carlo simulations when the true asymmetry data generating process is known. The Monte Carlo study is repeated for different sample sizes, different noise levels in the data and various differences in the asymmetric adjustment parameters to determine how the performance of the model selection procedure (i.e. Marginal likelihood and Information 
Criteria) varies with changes in sample size, stochastic variance and the level or strength of asymmetry.

In addition, the performance of the model selection algorithms is examined when the true asymmetric data generating process or the true model is relatively complex (i.e. complexity based on the number of asymmetric adjustment parameters).

There are many methods of choosing between competing models. This research emphasises the Bayesian approach to model selection which postulates the identification of the true model. The concept of the true model in model selection is inspired by advocates (Schwarz, 1978; Raftery 1986; Kass and Raftery, 1995; Kass and Wasserman, 1995; Albert and Chib, 1997, Koop, 2003, Geweke, 2005, Zellner, 1971). Advocates of the Bayesian approach argue that it provides a superior approach to model selection (Raftery, 1995). An alternative approach to model selection "the Frequentist approach" aims at finding the best approximating model to the unknown true data generating process and its applications draws from (Akaike, 1973; Bozdogan, 1987; Dayton 2003; Zucchini ${ }^{5}$ 2000). The debate on model selection continues on a variety of fronts. According to Box (1979), "All models are false, but some are useful". Fox (1997) argues that the idea of a true model is problematic; however hypothesis test assumes the existence of a true model. Alternatively, Wasserman (2000) affirms that the assumption of a true model is a useful tentative working hypothesis and proceeding under that hypothesis, the relative evidence in favor of competing theories or models can be derived. Although different model selection methods are based on different theoretical motivations and objectives, in a broader sense they all have the same aim of identifying good models even if they differ in their exact definition of a good model.

\subsection{Organisation of the thesis}

This thesis is structured into two main parts. Part I (chapters 1 and 2) presents the introduction and background theory of the research. Chapter 1 introduces and motivates the thesis, and sets out the objective of this research. Chapter 2 reviews previous studies and the theoretical concepts of price transmission within the context of the different test for asymmetry. Part II, which constitute chapter 3 to 5 presents the theoretical and empirical analysis of the study.

\footnotetext{
${ }^{5}$ See Zucchini (2000) for a fundamental discussions and clarification on difference between the frequentist and the Bayesian approach to model selection.
} 
Chapter 3 sets out the Bayesian theory on model comparison and choice in the context of Bayesian econometric methodology. The Bayesian analysis of the asymmetric price transmission models is developed within the context of a regression specification. In an empirical application involving artificial data via Monte Carlo Simulations, Chapter 4 provides model rankings for the different models on the basis of the concept of the marginal likelihood and Information Criteria. Using a Monte Carlo study, it establishes whether the Bayesian model selection criteria do help match the appropriate asymmetry test with the underlying data generating process (e.g. Asymmetric Error Correction Model data generating process). Additionally, the effects of different sample sizes, difference in the asymmetric adjustment parameters and error variance on the model selection procedures are evaluated.

In Chapter 5 we demonstrates that the conditions that affect the performance of the model selection methods in recovering the true asymmetric data generating process are also important in the power test of asymmetry. Chapter 6 concludes the study by providing methodological implications and directions for future research. 


\section{Theoretical Concept}

This section reviews previous studies and relates asymmetric price response to theories explaining such behavior. Empirical literature is discussed to demonstrate how the different econometric models which measure these asymmetrical behaviors detect asymmetries at different rates or culminates in different inferences and conclusions. A Bayesian viewpoint which provides a flexible and conceptually simple framework for comparing competing models is proposed to guide the rigorous comparison of the alternative methods of testing for asymmetry.

\subsection{Asymmetric Price Transmission}

Recent empirical studies analysing whether prices rise faster than they fall, have categorised the price dynamics into symmetric and asymmetric ${ }^{6}$ processes. Those processes for which the transmission differs accordingly to whether the prices are increasing or decreasing (i.e. asymmetric price transmission) are of keen interest. Most empirical research points to the fact that the price transmissions are asymmetric. Studies of various products and services, including gasoline, agriculture products, and bank deposit rates, all find that prices are more likely to rise to input price increases than they are to decrease in the wake of cost reduction. Peltzman (2000) significantly broadens the evidence for this asymmetrical price behavior. In a study of 77 consumer and 165 producer goods, he finds that on the average, the immediate response to a cost increase is at least twice the response to a cost decrease. This phenomenon presents an interesting empirical regularity that needs to be explained.

The issue of asymmetric price transmission continues to receive considerable attention in the economic literature for two prominent reasons. First, its presence is not in line with predictions of the conventional economic theory (e.g. perfect competition and monopoly) which postulates that under some regularity assumption (such as non-kinked convex or concave demand functions) prices should respond symmetrically to cost increases and cost reductions.

\footnotetext{
${ }^{6}$ By definition, asymmetry is an unreciprocal relationship between rises and falls in prices. e.g. Farm and retail prices
} 
The forgoing discussion is consistent with Peltzman (2000), who finds asymmetric price transmission to be the rule, rather than the exception and argues that it poses a real challenge to standard economic theorizing, since it does not predict or explain the existence of asymmetries.

Second, asymmetric price transmission is not only important because it points to gaps in the economic theory but also its presence has important welfare and hence policy implications (von Cramon-Taubadel and Meyer, 2004). It implies a different distribution of welfare than would be obtained under symmetry, since it alters the timing and size of welfare changes.

Additionally, the presence of asymmetric price transmission is often considered to be evidence of market failure (for example exercise of market power), von Cramon-Taubadel and Meyer (2004), signaling in addition to redistribution, the associated net welfare losses. Thus both redistribution and net welfare loss provide a prima facie case for policy intervention.

Von Cramon-Taubadel (1998) and von Cramon-Taubadel and Meyer (2004) provide a concise discussion of the definition of asymmetry in the context of price transmission with three main categorization criteria; a) asymmetry with reference to the speed and magnitude, b) asymmetry affecting vertical or spatial price transmission and, c) positive or negative asymmetry.

Notably, positive asymmetry defines a set of reactions in which any price movement that squeezes the margins is transmitted more rapidly than an equivalent that stretches the margin. Alternatively, asymmetric price transmission is negative, when any price movement that stretches the margin is transmitted more rapidly than those that squeeze the margin. Accordingly, Figure 1 below illustrates asymmetry with respect to the speed and magnitude with price $\mathrm{P}$ on the vertical axis and time $\mathrm{t}$ on the horizontal axis.

It is evident from Figure 1 below that a decrease in the input price $\left(p^{\text {in }}\right)$ takes three periods and is not fully transmitted to the output price $\left(p^{\text {out }}\right)$. While an increase in the input price $\left(p^{\text {in }}\right)$ takes only two periods and is fully transmitted to the output price ( $\left.p^{\text {out }}\right)$. 


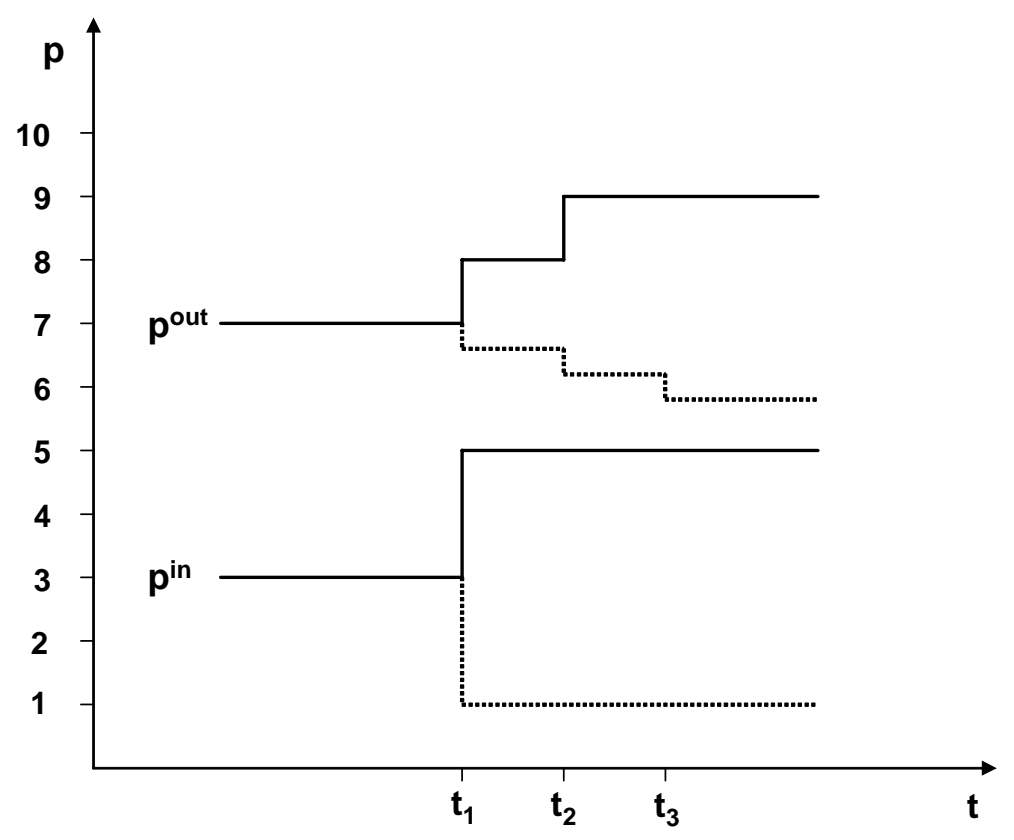

Figure 1: Types of Asymmetry (Speed and Magnitude).

\subsection{Rational for Asymmetric Price Transmission}

Several factors which culminate in asymmetric price transmission have been proposed in the literature. First, a commonly cited source of asymmetric price transmission is market power. (see for example Kinnucan and Forker 1987; Miller and Hayenga, 2001; McCorrisston, 2002; and Lloyd et al., 2003). Oligopolistic processors, for example, might react collusively more quickly to shocks that squeeze their margin than to shocks that stretch it, resorting in asymmetric short run transmission in an attempt to hide the exercise of market power behind the 'confusion' created by major shocks, processors could also react less completely to the shocks that stretch their margins leading to asymmetric long run transmission.

Similarly, asymmetric price transmission could result if traders in the local market believe that competitors will follow an increase in the local market prices as price in the central market rise, but that they will not respond to falling prices in the central market by granting an equivalent reduction. It is however important to mention that concentration is probably a necessary but certainly not a sufficient condition for the exercise of market power, as the theoretical and empirical evidence on the relationship between these two phenomena is inconclusive (Weaver et al., 1989; and Goodwin, 1994). Within the oligopoly context, both positive and negative asymmetries are conceivable depending on the market structure and conduct. In this regard, several studies of market power and asymmetry that focus on specific 
markets deserve to be mentioned. Borenstein et al. (1997) analysed vertical price transmission for crude oil to gasoline prices, and concluded that downward stickiness of retail prices for gasoline in an oligopolistic environment will lead to positive asymmetry.

Alternatively, Ward (1982) suggested that market power can lead to negative asymmetry if oligopolists are reluctant to risk losing market share by increasing output prices. Similarly Bailey and Brorsen (1989) considered firms facing a kinked demand curve that is either convex or concave to the origin. If a firm believes that no competitor will match a price increase but all will match a price cut (concave), negative asymmetry will result. Alternatively, if the firm conjectures that all firms will match an increase but none will match a price cut (convex), positive asymmetry will result. Hence, it is not clear a priori whether market power will lead to positive or negative asymmetry (Bailey and Brorsen, 1989).

Secondly, price asymmetry can be partly attributed to adjustment cost that arises when firms change their quantities and prices of inputs and outputs. Consequently, positive or negative asymmetric price transmission results if these costs are symmetric with respect to increase or decrease in quantities or prices. In an analysis of the US beef market, Bailey and Brorsen (1989) argued that firms may face different adjustment cost depending on whether prices are rising or falling. Subsequently, they noted that the competition between meat packers faced with a high fixed cost and excess capacity, for example, might result in farm prices that are bid up rapidly, in response to increased demand for meat products, but fall more slowly as demand weakens. Ward (1982) suggested that retailers of perishable products may be hesitant to raise prices for fear that they could end up holding spoiled stocks, leading to negative asymmetry. Heien (1980) disputes this assertion and notes that changing prices is less of a problem for perishable products than it is for those with a long shelf life, because for the latter, changing prices incur higher time cost and loss of good will. Thus, echoing the so called menu cost hypothesis proposed by Barro (1972), (i.e. a change in nominal price induces cost for example, the reprinting of price list or catalogues and the cost of informing market partners). Ball and Mankiw (1994) developed a model based on menu cost (the cost involved in changing nominal prices such as the cost of reprinting catalogues etc) in combination with inflation that leads to asymmetry. In this model, positive nominal input price shocks are more likely to lead to output price adjustment than negative price shocks. This is because in the presence of inflation, some of the adjustment made necessary by an input price reduction is automatically carried out by inflation, which reduces the real value of the margin. Thus in 
situations where firms face menu cost and inflation, shocks that bring upward price adjustment are rapidly responded to than those that reduce it, as inflation in this respect would have automatically affected some of the adjustment made necessary by the downward adjustment shocks (Kuran, 1983; Buckle and Carlson, 2000). In contrast to Bailey and Brorsen (1989), Peltzman (2000) makes a case for positive asymmetry affirming that it is easier for firm to disemploy inputs in the case of an output reduction than it is to recruit new inputs to increase output. This recruitment will lead to search cost and price premier increasing phases. Additional explanation for price asymmetry which has been proposed can not be considered directly under market power or adjustment cost.

Kinnucan and Forker (1987) suggested that asymmetry could result from government intervention, indicating that such political intervention can lead to asymmetric price transmission if it leads wholesalers or retailers to believe that a reduction in farm prices will only be temporary because it will only trigger government intervention, while an increase in farm prices is more likely to be permanent.

\subsection{Econometric Modeling of Asymmetric Price Transmission}

The modeling of asymmetric price transmission ${ }^{7}$ can be grouped into pre-cointegration and cointegration approaches (Meyer and von Cramon, 2004). The pre-cointegration and the cointegration approaches draw heavily from Houck (1977) and von Cramon (1998) respectively.

Drawing from Houck (1977) within the context of the pre-cointegration approaches, numerous authors have developed a test for asymmetric price transmission which is based on the segmentation of prices into increasing and decreasing phases (Kinnucan and Forker, 1987; Bailey and Brorsen, 1989; Zhang, Fletcher and Carley, 1995; Mohanty, Peterson and Kruse, 1995; Boyd and Brorsen, 1998). These different applications are considered as variants of the Houck's model and denoted by Houck's Approaches. These pre-cointegration approaches require the data to be stationary in order to avoid spurious regression. The cointegration approaches are motivated by the fact that the Houck's approaches are not consistent with cointegration between the price series involved. Emphatically, this is the motivation for the

\footnotetext{
${ }^{7}$ See von Cramon (1998, 2000 and 2004) for the detail discussions on the models of asymmetric price transmission.
} 
asymmetric error correction modeling (von Cramon-Taubadel, 1998, and von Cramon Taubadel and Loy, 1999).

\subsubsection{Houck's Specification}

Asymmetric Price Transmission has been tested in a wide variety of agricultural markets. Appel (1992) finds that both speed and degree of price transmission from the producer to the retail level for broilers in Germany is asymmetric. Boyed and Brorsen (1988) studied the US pork market and find no evidence of asymmetric price transmission. However, this result was challenged by Hahn (1990) who finds that prices at all levels of the US pork and beef marketing chains are more sensitive to price increasing shocks than to price decreasing shocks. Hansmire and Willett's (1992) indicated that farm-retail price transmission for New York state apples is asymmetric and Kinnucan and Forker (1987) came to the same conclusion regarding dairy product transmission in the United States. Pick et al. (1990) finds evidence that short-run but not long-run vertical price transmission on the US citrus market is asymmetric. Finally, Ward (1982) points to both short and long run asymmetries in vertical price transmission for fresh vegetable in the United States, while Zhang et al. (1995) noted that price transmission for pea nut to peanut butter prices in the US is asymmetric in the shortrun, but symmetric in the long-run.

Generally, each of these studies implements some variant of an econometric technique for estimating irreversibility that was introduced by Wolfram (1971) in response to work on irreversible supply reaction by Tweeten and Quance (1969). In investigating the relationship between an output price $P_{A}$ and input price $P_{B}$, Tweeten and Quance (1969) used an indicator variable to split the input price into two parts: one variable includes only increasing input prices $P_{B}^{+}$and another includes only decreasing input prices $P_{B}^{-}$. From this, two input price adjustments coefficients (i.e. $\beta_{1}^{+}$and $\beta_{1}^{-}$) can be estimated as specified below.

$$
P_{A, t}=\beta_{o}+\beta_{1}^{+} P_{B, t}^{+}+\beta_{1}^{-} P_{B, t}^{-}+\varepsilon_{t}
$$

Symmetric price transmission is rejected if the coefficients $\beta_{1}^{+}$and $\beta_{1}^{-}$are significantly different from one another. Based on Tweeten and Quance (1969), Wolffram (1971) proposes a variable splitting technique that explicitly includes first difference of prices in the equation 
to be estimated which was later modified by Houck (1977). Within the context of the Wolfram-Houck (W-H) method, the response of price $P_{A}$ to another price $P_{B}$ is estimated with the following equation.

$$
\Delta P_{A, t}=\beta_{o}+\beta_{1}^{+} \Delta P_{B, t}^{+}+\beta_{1}^{-} \Delta P_{B, t}^{-}+\varepsilon_{t}
$$

Where $\Delta P^{+}$and $\Delta P^{-}$are the positive and negative changes in $P_{B}$ respectively, $\beta_{o}, \beta_{1}^{+}, \beta_{1}^{-}$ are coefficients and $t$ is the current period. Numerous studies estimate a dynamic variant of the Houck's static model. Some analyst distinguish between short-run and long-asymmetries by introducing lagged terms in $\Delta P_{B, t}^{+}$and $\Delta P_{B, t}^{-}$into equation (2.2), in which case $\beta^{+}$and $\beta^{-}$become lag polynomials. Long-run symmetry is tested by determining whether the sums of the coefficients in these polynomials are identical. Ward (1982) extended the Houck's specification by including lags. While, Brorsen (1988) was the first to use lags to differentiate between magnitude and speed of transmission. Hahn (1990) attempts to generalize the methods discuss so far, referring to them as the pre-cointegration methods.

Other authors (e.g. Mohanty, Peterson and Kruse, 1995) take the sum of both sides of equation (2.2) to derive the following equation.

$$
\sum_{t=1}^{T} \Delta P_{A, t}=\alpha_{o}+\alpha_{1} \sum_{t=1}^{T} \Delta P_{B, t}^{+}+\alpha_{2} \sum_{t=1}^{T} \Delta P_{B, t}^{-}+\varepsilon_{t}
$$

which can be rearranged as follows:

$$
P_{A, t}-P_{A, 0}=\alpha_{o}+\alpha_{1} P_{B, t}^{U P}+\alpha_{2} P_{B, t}^{D O W N}+\varepsilon_{t}
$$

where $P_{B, t}^{U P}$ is the sum of all positive changes in price $\mathrm{B}$ and $P_{B, t}^{D O W N}$ is the sum of all negative changes in price $\mathrm{B}$. A formal test for symmetry using an $\mathrm{F}$ test or $\mathrm{t}$-statistic is rejected when the coefficients $\alpha_{1}$ and $\alpha_{2}$ are unequal.

The Houck model is sometimes used without adequate regards to time series properties of the data. Von Cramon-Taubadel (1998) has demonstrated that the model is fundamentally incompatible with cointegration between two price series. 
In order to demonstrate this point, von Cramon-Taubadel (1998) considers two

processes, $P_{A}$ and $P_{B}$, and the model below as previously defined in equation 2.2 and 2.3:

$\sum \Delta P_{A, t}=\beta_{o}+\beta_{1}^{+} \sum \Delta P_{B, t}^{+}+\beta_{1}^{-} \sum \Delta P_{B, t}^{-}+\varepsilon_{t}$

which can be reparametrized using the identity:

$\sum \Delta P_{B, t}^{+}+\sum \Delta P_{B, t}^{-} \equiv P_{B, t}-P_{B, 0}$

to yield:

$P_{A, t}-P_{A, 0}=\beta_{o}+\beta_{1}^{+} \sum \Delta P_{B, t}^{+}+\beta_{1}^{-}\left(P_{B, t}-P_{B, 0}-\sum \Delta P_{B, t}^{+}\right)+\varepsilon_{t}$

or:

$P_{A, t}=\left(P_{A, 0}+\beta_{o}+\beta_{1}^{-} P_{B, 0}\right)+\beta_{1}^{-} P_{B, t}+\left(\beta_{1}^{+}-\beta_{1}^{-}\right) \sum \Delta P_{B, t}^{+}+\varepsilon_{t}$

This reparametrization of equation (2.5) was proposed by Ward (1982) who tests whether the coefficient $\left(\beta_{1}^{+}-\beta_{1}^{-}\right)$differs from 0 in order to test whether price transmission is asymmetric. Von Cramon-Taubadel (1998) asserts that the estimation of equation 2.8 can lead to four basic results depending on the significance of the term $\left(\beta_{1}^{+}-\beta_{1}^{-}\right)$and the stationarity of the error term $\varepsilon_{t}$ :

- Case 1: $\beta_{1}^{+}-\beta_{1}^{-} \neq 0$ (asymmetry) and $\varepsilon_{t}$ is $\mathrm{I}(0)$

- Case 2: $\beta_{1}^{+}-\beta_{1}^{-}=0$ (symmetry) and $\varepsilon_{t}$ is I(1)

- Case 3: $\beta_{1}^{+}-\beta_{1}^{-} \neq 0$ (asymmetry) and $\varepsilon_{t}$ is I(1)

- Case 4: $\beta_{1}^{+}-\beta_{1}^{-}=0$ (symmetry) and $\varepsilon_{t}$ is $\mathrm{I}(0)$ 
Case 1 implies that $P_{A}, P_{B}$ and $\sum \Delta P_{B, t}^{+}$are cointegrated, which precludes cointegration between $P_{A}$ and $P_{B}$ alone. Case 2 and 3 are spurious regressions (Granger and Newbold, 1974), while case 4 implies that $P_{A}$ and $P_{B}$ are cointegrated. Notably, if the Houck method points to asymmetry, then either the results reflect spurious regression (Case 3), or the prices in question are not cointegrated (Case 1).

\subsubsection{The Asymmetric Error Correction Representation}

Fundamentally, the asymmetric error correction model (ECM) approach is motivated by the fact that all the variants of the aforementioned Houck approach discussed above are not consistent with cointegration between the price series. If the prices $P_{A}$ and $P_{B}$ are cointegrated, then an error correction representation exists (Engle and Granger, 1987). Granger and Lee (1989) propose a modification to the error correction representation that makes it possible to test for asymmetric price transmission between cointegrated variables. This involves a Wolffram-type segmentation of the error correction term into positive and negative components.

The first attempt to draw on cointegration technique in testing for asymmetry in vertical price transmission is von Cramon-Taubadel and Fahlbusch (1994) and later elaborated by von Cramon-Taubadel and Loy (1996) and von Cramon-Taubadel (1998). They suggested that in the case of cointegration between the price series, an error correction model extended by the incorporation of asymmetric adjustment terms provides a more appropriate specification for testing for asymmetric price transmission. In effect, when equation (2.9) is estimated and the test proves that it is not a spurious regression, then $P_{A}$ and $P_{B}$ is referred to as being cointegrated, and equation (2.9) can be considered as an estimate of the long-run relationship between them.

$$
P_{A, t}=\beta_{o}+\beta_{1} P_{B, t}+\varepsilon_{t}
$$

The Error Correction Model (ECM) then relates changes in $P_{A}$ to changes $P_{B}$ as well as the so called Error Correction Term (ECT), the Lagged residuals derived from estimation of equation (2.9). The ECT measures the deviation from the long-run equilibrium between the 
$P_{A}$ and $P_{B}$, and including it in the ECM allows $P_{A}$ not only to respond to changes in $P_{B}$ but also to correct any deviations from the long-run equilibrium that may be left over from previous periods. Splitting the ECT into positive and negative component (i.e. positive and negative deviation from the long-run equilibrium- $E C T^{+}$and $E C T^{-}$) makes it possible to test for asymmetric price transmission.

$$
\Delta P_{A, t}=\beta_{\mathrm{o}}+\beta_{1} \Delta P_{B, t}+\beta_{2}^{+} E C T^{+}{ }_{t-1}+\beta_{2}{ }^{-} E C T^{-}{ }_{t-1}+\beta_{3} \Delta P_{B, t-1}+\beta_{4} \Delta P_{A, t-1}+\varepsilon
$$

Von Cramon-Taubadel and Loy (1996) also segmented the contemporaneous response term in equation (2.9). This leads to the following specification in which contemporaneous and shortrun response to departures from the cointegrating relation are asymmetric if $\beta_{1}^{+} \neq \beta_{1}^{-}$and $\beta_{2}^{+} \neq \beta_{2}^{-}$respectively:

$$
\Delta P_{A, t}=\beta_{\mathrm{o}}+\beta_{1}^{+} \Delta P_{B, t}^{+}+\beta_{1}^{-} \Delta P_{B, t}^{-}+\beta_{2}^{+} E C T^{+}{ }_{t-1}+\beta_{2}^{-} E C T^{-}{ }_{t-1}+\beta_{3} \Delta P_{B, t-1}+\beta_{4} \Delta P_{A, t-1}+\varepsilon
$$

Noticeably, equation 2.11 is equivalent to the Houck approach given by equation 2.2, except that equation 2.11 also contains $\beta_{2}^{+} E C T^{+}{ }_{t-1}, \beta_{2}{ }^{-} E C T^{-}{ }_{t-1}, \beta_{3} \Delta P_{B, t-1}, \beta_{4} \Delta P_{A, t-1}$. Thus in effect the asymmetric ECM with complex dynamics nests the Houck's model in first difference or has the structures of the Houck's model.

The discussion so far has emphasised the Granger and Lee asymmetric error correction model implemented in von Cramon-Taubadel (1998). Some studies employ variants of these approaches. For instance a recent approach proposed by Chavas and Mehta (2004) appears to nest a variety of earlier approaches.

Numerous price transmission studies implements von Cramon-Taubadel and Loy (1996) testing procedure for asymmetric price transmission or some variants of their proposed ECM approach. For example von Cramon-Taubadel and Loy (1996) use an ECM to study the spatial price transmission on world wheat markets. Similarly, Capps and Sherwell (2007) analysed the behaviour of spatial test of asymmetric price transmission according to the Von Cramon-Taubadel and Loy ECM approach and the conventional Houck approach. Scholnick (1996) also uses an asymmetric ECM to test for asymmetric adjustment of interest rates, while Borenstein et al (1997) employ an ECM specification where the error correction terms are not 
segmented. Von Cramon-Taubadel (1998) demonstrates that transmission between producer and wholesale pork prices in northern Germany is asymmetric. Balke et al. (1998) and Frost and Bowden (1999) also employ variants of the asymmetric ECM. FAO (2003) provide a review of the application of time series techniques (cointegration, ECM) in testing market integration and price transmission for a number of cash and food crop markets in developing countries.

\subsubsection{Models with a Threshold}

Following the threshold approach introduced by Tong (1983), it is possible to consider an intuitively appealing type of ECM in which deviation from the long-run equilibrium between $P_{A}$ and $P_{B}$ will lead to a price response if they exceed a specific threshold level. Several studies measuring asymmetric price transmission using the threshold approach estimates variants of the following simplified equation.

Standard Threshold Cointegrated Model

$$
\Delta P_{A, t}=\beta_{\mathrm{o}}+\beta_{1} \Delta P_{B, t}+\beta_{2}{ }^{+} E C T^{+}{ }_{t-1}+\beta_{2}^{-} E C T^{-}{ }_{t-1}+\varepsilon_{t}
$$

Given a threshold $(\gamma)$, where: $E C T^{+}{ }_{t-1}>\gamma$ and $E C T^{-}{ }_{t-1} \leq \gamma$

The Error Correction Term (ECT) is segmented into $E C T^{+}$and $E C T^{-}$according to whether it is greater or less than a defined threshold value respectively. Detailed discussion on the threshold modeling is provided in numerous studies including (Enders 2004, pp. 393-397; Balke and Fomby 1997 and Tsay 1998). Balke and Fomby (1997) presented a model that allows for non linear adjustment to equilibrium by introducing the concept of threshold cointegration. The relationship between symmetry and threshold is systematically developed in von Cramon-Taubadel and Meyer (2004). The authors note that thresholds allow for different types of asymmetry.

The first type refers to a two symmetric threshold model (i.e. three regime model) with asymmetric responses in the outside regimes, reflecting asymmetry with respect to speed of transmission. In contrast, a two symmetric threshold model with three regimes and symmetric responses in the outside regimes need not be asymmetric. On the other hand, a one threshold model is asymmetric if the threshold parameter $(\gamma)$ differs from zero. 
The second type of asymmetry refers to the fact that the two thresholds need not be equal. If this type of asymmetry holds the deviations in the positive and negative directions must reach a different magnitude before a price response is triggered.

Following the development of the threshold model, a number of applications have estimated asymmetric adjustments using threshold error correction models. Abudulai (2002) draws on Enders and Granger (1998) to test for asymmetric price transmission in a methodology in which the threshold parameter $(\gamma)$ is set to zero. Alternatively, Godwin and Harper (2000) and Goodwin and Piggott (2001) use a grid search to find optimal thresholds in price transmission analysis. Hansen and Seo (2002) develop a test for the significance of a single threshold in an error correction model where the ECT is segmented not according to whether it is greater or less than zero but rather according to whether it is greater or less than a threshold value that might differ from zero. In an empirical application, Aguero (2004) uses a threshold error correction model to estimate price adjustments under risk in the Peruvian agricultural markets.

\subsection{Empirical Overview of the Different Asymmetry Methods}

The review of empirical application is based on thorough literature survey of asymmetric price transmission which draws heavily from von Cramon and Meyer (2004). The survey documentation in von Cramon-Taubadel and Meyer (2004) consist of 40 publications estimating asymmetric price transmission in samples of different products including fuel and gasoline, interest rates, and agricultural products.

Most of these applications are based on monthly and weekly price data while daily, fortnightly and quarterly data are each used once. Nearly half of the test for asymmetric price transmission employs some type of pre-cointegration procedure (i.e. 19 out of 40). Post cointegration methods such as the error correction mechanisms and the threshold approaches are employed in 11 publications (i.e. 4 ECM and 7 threshold applications). The remaining studies implement a variety of other approaches. The table below presents the outcome of a Meta analysis based on the results of all published individual tests derived in von CramonTaubadel and Meyer (2004). 
The 40 publications yield 205 individual test of asymmetric price transmission since several papers cover more than one product. Out of these, 93 apply a pre-cointegration test based on first differences (Houck 1977; Boyed and Brorsen, 1988). 53 apply a pre-cointegration approach based on recursive sums of the first differences (Wolfram, 1971; Houck, 1977; and Ward, 1982). 31 apply asymmetric error correction model (von Cramon-Taubadel, 1998; Granger and Lee, 1989). And 28 apply either threshold or other techniques (Tong, 1983; Godwin and Piggot, 2001; Hansen and Seo, 2002; Meyer and von Cramon-Taubadel, 2005).

\section{Table 1: Asymmetry Test of the Different Econometric Models}

\begin{tabular}{|l|l|l|l|l|l|l|}
\hline & \multicolumn{7}{|c|}{ Test } & Methods \\
\hline & $\begin{array}{l}\text { All } \\
\text { Methods }\end{array}$ & $\begin{array}{l}\text { Methods } \\
\text { using } \\
\text { first } \\
\text { difference }\end{array}$ & $\begin{array}{l}\text { Methods } \\
\text { using } \\
\text { summed } \\
\text { difference }\end{array}$ & $\begin{array}{l}\text { ECM } \\
\text { Methods }\end{array}$ & $\begin{array}{l}\text { Threshold } \\
\text { Methods }\end{array}$ & $\begin{array}{l}\text { Misc } \\
\text { Methods }\end{array}$ \\
\hline Total cases & 205 & 93 & 53 & 31 & 10 & 18 \\
\hline $\begin{array}{l}\text { Symmetry } \\
\text { maintained }\end{array}$ & 106 & 30 & 40 & 17 & 2 & 17 \\
\hline Symmetry rejected & 99 & 63 & 13 & 14 & 8 & 1 \\
\hline $\begin{array}{l}\text { Symmetry rejected } \\
\text { (\%) }\end{array}$ & 48 & 68 & 25 & 45 & 80 & 6 \\
\hline
\end{tabular}

Source: Meyer and von Cramon-Taubadel (2004, pp. 22)

Inferring from the reviewed literature (see von Cramon-Taubadel and Meyer, 2004), it is increasingly evident that the different methods appear to lead to different rate of rejection of the null hypothesis of symmetry. The fact that the literature to date contains no rigorous comparison and analysis of the strengths and weaknesses of the available methods is a hurdle to the advancement of asymmetric price transmission analysis. If researchers knew which method is appropriate, they could focus on this and accumulate insight and knowledge more rapidly in the future.

Von Cramon-Taubadel and Meyer (2004) noted that the available methods are not all simply reparameterisation of one another and that they can not all be equally appropriate in all cases. Emphatically, the survey by von Cramon-Taubadel and Meyer (2004) showed that the different methods detect asymmetry at different rates (but this could be because different methods applied to different data in each case). Subsequently, a simple experiment with simulated data implemented in this research and detailed in the appendix I produces a similar result consistent with the findings of the survey by von Cramon-Taubadel and Meyer (2004) that the different methods detect asymmetry at different rates. 
Alternatively, using a conventional Houck's model and von Cramon-Taubadel and Loy ECM approach in an empirical analysis of the U.S. markets, Capps and Sherwell (2007) conclude that differences in inferences are possible and conclusions derived from the ECM approach in the Seattle and St Louis milk markets was not supported by the Houck's model in the same market with the same data. Fundamentally, the F- test associated with the null hypothesis that retail prices respond symmetrically to increases and decreases in farm prices is not rejected in the case of Houck's model for above milk markets. In contrast, the hypothesis of symmetry is rejected in the ECM approach.

With different econometric models of asymmetric price transmission detecting asymmetry at different rates or culminating to differences in inferences and conclusion, it remains imperative to select one model from the set of competing models that best captures the true underlying asymmetric data generating process.

In addressing these issues of model comparison and selection, Bayesian approach to hypothesis testing which provides a set of tools for identifying a true asymmetric data generating process from alternative competing models is adopted. Subsequently the conceptual framework guiding this research draws principally from the Bayesian Inference on model selection and conceptualizes that the alternative asymmetric price transmission models can be compared on the basis of measures of model fit such as the marginal likelihood ( Gelfand and Dey, 1994) and information criteria (Koop, 2003). The intuition underpinning the concept of the marginal likelihood is that models should be evaluated on the probability they assign the data or on the probability that they generated the data. In sections 3.2, 3.5 and 3.8 the definitions and development of the concept of the marginal likelihood and information criteria are detailed.

The adoption of Bayesian approach to model selection is further motivated by the fact that the procedures are conceptually simple to understand and practically feasible to implement for any number of competing price transmission models. Moreover Bayesian methods do not require competing models to be nested in order to make a rigorous comparison. Importantly, model rankings or evidence in favour of competing theories can be derived as a basis for comparison and selection. 
Fundamentally, the different asymmetry tests are based on different underlying asymmetrydata generating processes. Subsequently, if the Bayesian criteria do help 'match' the appropriate test to an underlying data generating process in this research, then this would help practitioners in the future to select the right test to apply when confronted with real data for which they do not know the data generating process. In effect, researchers can employ the Bayesian criteria capable of identifying the true asymmetric price transmission model from this research for addressing model selection problems. Additionally, this research sheds light on the relative performance of the model selection methods in an asymmetric price transmission modeling framework for which no studies has been undertaken. In light of these assertions among others, this research addresses the methodological issue of rigorous comparison of these alternative models of asymmetric price transmission from a Bayesian perspective.

\subsection{Summary and Conclusions}

This chapter reviewed the theoretical concepts of price transmission within the context of the different test for asymmetry. This included discussions on asymmetry in terms of economic theory, examination of the rationale of this asymmetrical behavior in price transmission and the applicable econometric models.

Recent empirical studies analysing whether prices rise faster than they fall and vice versa have applied variants of different econometric models to test price asymmetry as a working hypothesis. The empirical review of these different asymmetry test points to the fact that these alternative methods detect asymmetry at different rates or culminates to differences in inferences and conclusions and emphasised the need to address this methodological problem of having many alternative models of testing for asymmetry. In effect, the methodological question of which models fit or explains a given set of data better is intriguing since the available methods are not all simply reparameterisation of one another and that they can not all be equally appropriate in all cases. Moreover, the different approaches may lead to differences in inference and conclusions (Capps et al 2007, von Cramon-Taubadel et al. 1999).

The performance of the Bayesian Methods to discriminate between different asymmetric price transmission models and the ability of the Bayesian criteria to clearly identify the underlying 
asymmetry data generating process for the simulated data in a Monte Carlo study is critical to addressing the problem of model selection between competing asymmetric price transmission models when the researcher does not know the true data generating process.

The straight forward conceptual framework guiding this study draws principally from the Bayesian theory on model selection which provides a flexible and conceptually simple framework for rigorous comparison of competing models. This research conceptualizes that alternative models can be compared on the basis of the concept of the marginal likelihood and deviance among other Bayesian measures of model fit. Using these criteria, model rankings are derived as a basis for comparison and selection. In subsequent chapters, these perspectives are developed and implemented. 


\section{Analysing Asymmetric Price Transmission using Bayesian Econometric Methodology}

An introduction to Bayesian inference is derived using the laws of conditional probability and the Bayes' Theorem. Essentials of a Bayesian analysis such as the prior distribution, the likelihood function and the posterior distribution are discussed. Methodologically, this research introduces and develops selection criteria that can apply jointly across competing asymmetric price transmission methods or model specifications during model selection. This entails drawing from the Bayesian theory on model selection, and comparing the different models of asymmetric price transmission on the basis of the marginal likelihood and deviance.

Subsequently, Bayesian approach to modeling asymmetric price transmission is developed in this section. Mathematical derivations and theoretical analysis are applied to deduce the conjugate prior distribution for the regression specification. The theoretical analysis further illustrates how data information is combined with prior information to obtain the posterior inference and the marginal likelihood of the price transmission regression model. First, the Bayesian analysis of the models is developed by solving the integrals in the marginal likelihood analytically (via analytical integration) using a natural conjugate prior. Second, the alternative analytical method of computing the marginal likelihood with no prior knowledge is also developed. Additionally, the Bayesian Information Criteria and Draper's Information Criteria are introduced as alternative procedures for comparing the competing price transmission models. The Bayesian methodology has the compelling benefit of providing a rigorous model comparison for various competing models with a conceptually simple framework.

\subsection{Bayesian Estimation}

Bayesian theory is briefly reviewed in this section. Detail account is provided in Koop (2003), Gill (2002), Gelman (2004), Lancaster (2004) and Geweke (2005). From a Bayesian perspective, there are two types of quantities: known and unknown. The Bayesian framework aims to use the known quantities along with a specified parametric expression to make inferential statements about the unknown quantities or parameters of interest. Bayesian estimation treats the unknown parameters as random variables and adopting subjective view 
of probability, it argues that our uncertainty about anything unknown can be expressed using the rules of probability (see Poireir, 1995; and Koop, 2003).

Let $p($.$) denote probability and y$ be a vector of $n$ data observations, assumed to be dependant on a vector of $\mathrm{k}$ unknown parameters $\theta$. Before any data are observed, our belief and uncertainties about $\theta$ is represented by a prior distribution (i.e. prior probability density $p(\theta))$. The probability model is specified by the likelihood $p(y \mid \theta)$, which is the probability of observing the data $y$ given that $\theta$ is the true parameter vector. Having observed the data, we update our belief about $\theta$ using the Bayes' Theorem to obtain the posterior distribution of $\theta$ given the data $y$.

$$
p(\theta \mid y)=p(y \mid \theta) p(\theta) / p(y)
$$

Using the law of conditional probability $p(y)=\int p(y \mid \theta) p(\theta) \partial \theta$ denotes a normalising constant. Since it is a constant not depending on $\theta$, the Bayes' Theorem can be rewritten as

$$
p(\theta \mid y) \propto \quad p(y \mid \theta) p(\theta)
$$

Thus the posterior distribution which is of fundamental interest in a Bayesian analysis is proportional to the likelihood times the prior distribution. The posterior distribution $p(\theta \mid y)$ is the $\mathrm{k}$ dimensional, but can often be simplified to focus on individual parameters such as $\theta_{i}$ (i.e. the component of $\theta$ ) by analytically or numerically integrating out the other components so that

$$
p\left(\theta_{1} \mid y\right)=\int p(\theta \mid y) \partial \theta_{2} \partial \theta_{3} \ldots . . \partial \theta_{k}
$$

The marginal distribution of $\theta_{1}$ contains all the information needed to make inferences about $\theta_{1}$ and may be summarized in measures such as its moments. The normalizing integral $p(y)$ may also be computed from this marginal distribution and used in model comparison exercise.

The use of likelihood and a prior in Bayesian analysis allow strong prior knowledge from theory or previous studies to build into the estimation, but in the present study we wish to express a weak prior knowledge or ignorance which is consistent with most Bayesian econometric applications. This is done in the context of a natural conjugate prior (i.e. a prior which has the same functional form as the likelihood function), and priors which are diffuse 
relative to the likelihood. In effect the priors used ensure that data information is predominant over prior information. A detailed discussion is presented in Zellner (1971), Jeffereys (1961), Kass and Wasserman (1995), Geweke (2005) and Koop (2003).

The theoretical concepts outlined above suggests that Bayesian econometrics requires a prior and likelihood to form the posterior which forms the basis for all inference about unknown parameters in the model. It is important to be explicit about which model is under consideration since this research is interested in alternative models. Suppose we have $m$ different models, $M_{i}$ for $i=1 \ldots \ldots . . . m$, which all seek to explain y. $M_{i}$ depends on parameters $\theta^{i}$ and the posterior distribution of parameters calculated using $M_{i}$ is written as:

$$
p\left(\theta^{i} \mid y, M_{i}\right)=p\left(y \mid \theta^{i}, M_{i}\right) p\left(\theta^{i} \mid M_{i}\right) / p\left(y \mid M_{i}\right)
$$

Unlike equation 3.1, the notation makes it clear that we now have a posterior $p\left(\theta^{i} \mid y, M_{i}\right)$, a likelihood $p\left(y \mid \theta^{i}, M_{i}\right)$ and a prior $p\left(\theta^{i} \mid M_{i}\right)$ for each model. The marginal likelihood of each model $p\left(y \mid M_{i}\right)$ is obtained by integrating both sides of 3.4 with respect to $\theta^{i}$, using the fact that $\int p\left(\theta^{i} \mid y, M_{i}\right) \partial \theta^{i}=1$ (since probability density functions integrates to one) and rearranged to obtain:

$$
p\left(y \mid M_{i}\right)=\int p\left(y \mid \theta^{i}, M_{i}\right) p\left(\theta^{i} \mid M_{i}\right) \partial \theta^{i}
$$

Noticeably, the marginal likelihood is the integral of the likelihood function with respect to the prior density Koop (2003 pp. 3-4). Alternatively, the marginal likelihood may be defined as the probability of the data average over the entire range of parameter values and the logarithm of the marginal likelihood of model $M_{i}$ becomes:

$\log p\left(y \mid M_{i}\right)=\log \int p\left(y \mid \theta^{i}, M_{i}\right) p\left(\theta^{i} \mid M_{i}\right) \partial \theta^{i}$

Subsequent sections detail the foregoing discussion. 


\subsection{Bayesian Model Comparison and Choice}

The previous discussion assumed that there is a set of $M_{i}$ competing models under examination and estimation as briefly illustrated using equation 3.4. Assuming the data $y$ is to be used to compare two models $M_{1}$ and $M_{2}$ with the parameter vectors $\theta_{1}$ and $\theta_{2}$. Using Bayesian analysis, the models can be compared on the basis of the marginal likelihood they attain. Cox (1961) and Geweke (2005) asserts that within the context of Bayesian model choice, model nesting is not required in order to make comparisons, thus addressing a major deficiency with classical approaches. Subsequently numerous empirical applications have compared variants of models conveniently using the concept of the marginal likelihood as the basis of comparison (McCulloch and Rossi, 1992; Koop and Potter, 1999; Bos, 2002 and Geweke 2005, pp 154-160).

When models are estimated in a classical manner, they can be compared on the basis of the likelihood they attain. The likelihood function is evaluated in the points indicated by the parameter estimates, often at the location of the maximum likelihood. Within the Bayesian framework, no one parameter vector characterizes the fit of the model. Instead, based on the likelihood and the prior, the full posterior distribution of the parameter is derived. A characteristic of the fit of the model $M$ is in this case the expected marginal likelihood $p(y \mid M)$, where the expectation is taken over the likelihood $p(y \mid \theta, M)$ with respect to the prior distribution $p(\theta)$ of the parameters (See equation 3.5).

The marginal likelihood of each model can be obtained by integrating over $\theta$ of the model. Thus, the marginal likelihood is obtained by integration across the range of $\theta$, in contrast to maximization that is developed in the maximum likelihood analysis. The marginal likelihood forms the main ingredients of the Bayes' factor which is useful for model comparison (Kass and Raftery, 1993). Koop (2003) demonstrates the usefulness of the marginal likelihood in comparing linear and non-linear models. Hepple (2004) suggests that the differences between Bayesian Information Criteria (BIC) and explicit Bayesian analysis using the marginal likelihood lies in what the two perspectives are measuring: BIC compares the height of the likelihood at the maxima, whereas the marginal likelihood compares the specification in terms of the whole integral across the marginal posterior. 


\subsection{Implementing Bayesian Model Choice in Practice}

In an analysis of several candidate models, one can either choose a single model or average over all the models. The Bayesian methods provide a set of tools for these model selection problems with the provision of numerical measures of the relative evidence in favor of competing theories. The practical question is how to implement the theory. The marginal likelihood for each model lies at the heart of the theory and can be computed directly or must be approximated or simulated. Geweke (2005) points out that simulation of the marginal likelihood is not a trivial procedure.

\subsection{Bayesian Analysis of the Standard Regression Model}

The Bayesian modeling of the standard regression model entails combining data information (i.e. likelihood) with prior information to obtain the posterior distribution which forms the basis for all inference about unknown parameters in the model. This completes parameter estimation in the Bayesian analysis and forms the basis for the model comparison exercise.

With the sample from the posterior distribution almost any quantity of interest can be estimated. For example, integrating across the posterior distribution with respect to the unknown parameters in the model yields the Marginal Likelihood.

Throughout this thesis, standard Bayesian econometrics notation exemplified in Koop (2003 pp. 15-87) and Geweke (2005 pp. 21-71) are adopted. Bars under parameters (e.g $\underline{\beta}$ ) are used to denote parameters of a prior density and bars over parameters (e.g. $\bar{\beta}$ ) are used to denote parameters of a posterior density. With regards to distributions used $N(), G()$ and $N G($ ) denotes normal, gamma and normal-gamma distributions respectively. Notably, $\hat{\beta}, s^{2}, v$ are the Ordinary Least Squares (OLS) estimator for $\beta$, standard error and degrees of freedom. The prior mean and variance of $\beta$ in the normal-gamma prior are $\underline{\beta}$ and $\underline{V}$, and the prior mean and degrees of freedom of the error precision $h$ are $\underline{s^{2}}$ and $\underline{v}$ respectively. With regards to hyper parameters of the posterior distribution, $\bar{\beta}$ and $\bar{V}$ defines the posterior mean and variance of $\beta$ and the posterior mean and degrees of freedom of the error precision $h$ is $\bar{s}$ and $\bar{v}$. 
The definitions of the notations are detailed in the proceeding discussions. And p (.) denotes probability as previously defined. With regards to matrix notation, || refers to the determinant of a matrix. For example, $\left|\overline{V_{j}}\right|$ and $\left|\underline{V}_{j}\right|$ are the determinant matrices of $\overline{V_{j}}$ and $\underline{V}_{j}$ (i.e. posterior and prior variances) respectively. A single vertical bar $\mid$ represents given, for example $p(y \mid \theta)$ denotes the probability of observing the data $y$ given $\theta$ as previously mentioned. The data sample size is defined by $n$ and $\mathrm{M}$ denotes a model.

\subsubsection{The Regression Model of Asymmetric Price Transmission Analysis}

Fundamentally, all common asymmetric price transmission models reviewed in section 2.2 of chapter 2 (i.e. Houck's models, Error correction models and Threshold models) can be represented as a standard regression model as specified below:

$$
y=X \beta+\varepsilon_{i} \quad \quad \varepsilon_{i} \sim \operatorname{iidN}\left(0, \sigma^{2}\right)
$$

Importantly, equation 3.7 above represents the standard asymmetric price transmission models with a response variable $\mathrm{y}$ and independent variables in $\mathrm{X}$ defined to include asymmetric adjustment terms. Subsequently, the Bayesian analysis of the standard regression model is valid for all common asymmetric price transmission models.

The Bayesian analysis of the error correction model is presented here as an illustration since the Bayesian analysis of the standard regression model is applicable to all common price transmission models.

In the presence of cointegration between two variables or price levels, Engle and Granger (1987) suggest that the error correction mechanism exist. Subsequently, the Error Correction Model relates changes in the dependent variable to the independent variable as well as the Error Correction Term (ECT). That is the lagged residual from the cointegration relationship between the two variables or price levels. The ECT which measures the deviations from the long-run equilibrium between the two prices is splitted into positive and negative component to allow testing for asymmetry. The standard asymmetric ECM can be specified as follows:

$$
\Delta P_{A, t}=\beta_{o}+\beta_{1} \Delta P_{B, t}+\beta_{2}{ }^{+} E C T^{+}{ }_{t-1}+\beta_{2}{ }^{-} E C T^{-}{ }_{t-1}+\varepsilon
$$


Where deviations from the cointegrating ${ }^{8}$ relationship between $P_{A t}$ and $P_{B t}$ (i.e ECT) is

Given by:

$E C T=P_{A t}-\beta_{0}-\beta_{1} P_{B t}$

Using matrix notation, the above equation becomes:

$$
\Delta P_{A, t}=\left[\begin{array}{llll}
1 & \Delta P_{B, t} & E C T^{+}{ }_{t-1} & E C T^{-}{ }_{t-1}
\end{array}\right]\left[\begin{array}{c}
\beta_{0} \\
\beta_{1} \\
\beta_{2}^{+} \\
\beta_{2}^{-}
\end{array}\right]+\varepsilon_{i}
$$

Where $\varepsilon_{i} \sim \operatorname{iidN}\left(0, \sigma^{2}\right)$

Denoting $\mathrm{y}=\Delta P_{A, t}, \quad X=\left[\begin{array}{llll}1 & \Delta P_{B, t} & E C T^{+}{ }_{t-1} & E C T^{-}{ }_{t-1}\end{array}\right]$ and $\beta=\left[\begin{array}{c}\beta_{0} \\ \beta_{1} \\ \beta_{2}^{+} \\ \beta_{2}^{-}\end{array}\right]$

The conditional distribution of $y$ given the vector of explanatory variables in $X$ can be represented by a standard regression model as specified in equation 3.7. Subsequently, all that follows is the implementation of the Bayesian analysis of the standard regression model which draws heavily from LeSage (1990), Bikker (1998), Koop ${ }^{9}$ (2003), and Congdon (2003) among others.

The Bayesian analysis of the error correction model implements a two step procedure. First the cointegration relationship is first estimated using the Granger methodology (i.e. equation 3.9). Second, the error correction term derived is included as an explanatory variable in the error correction model (i.e. equation 3.8) and estimated in a Bayesian fashion. This entails combining the data information with prior information to obtain the posterior distribution and the marginal likelihood within the context of the Bayesian analysis of the standard regression model.

\footnotetext{
${ }^{8}$ The cointegration relationship (i.e. ECT) in this research is estimated by the Engle Granger Methodology. An alternative is to use the Johansen Methodology.

${ }^{9}$ I benefited from suggestions from Prof. Dr. Koop, the author of Bayesian Econometrics (2003).
} 
The two-step procedure applied in the Bayesian modeling of the ECM was initially proposed and published in LeSage (1990) and subsequently implemented by various authors. This research departs ${ }^{10}$ from previous studies by modeling asymmetries in the short run adjustments to equilibrium within the context of a Bayesian analysis and fundamentally providing a Bayesian framework for investigating the problem of identifying a true asymmetric data generating process during model selection in price transmission analysis. Essentially, it derives the analytical marginal likelihood and other measures of model selection of the asymmetric price transmission model.

In summary all common asymmetric price transmission models can be represented by a standard regression model and the Bayesian inference of the standard regression model is valid for the common price transmission models. Subsequently analytical results of the Bayesian analysis of the price transmission models are derived as follows.

\subsubsection{The Likelihood Function}

From the asymmetric price transmission model specified above in equation 3.10 , it can be inferred that the errors of the model are assumed to be multivariate normal. Using this definition of multivariate normal density, the likelihood function can be written as:

$$
p(y \mid \beta, h)=\frac{h^{n / 2}}{(2 \pi)^{n / 2}}\left\{\exp \left[-\frac{h}{2}(y-X \beta)^{\prime}(y-X \beta)\right]\right\}
$$

Where the precision $h$ is defined as $h=\frac{1}{\sigma^{2}}$

It is convenient to write the likelihood function in terms of $\hat{\beta}, s^{2}, v$, the ordinary least squares estimator for beta, standard error and degrees of freedom respectively, Koop ( 2003 pp 16-36). The intuition is to rewrite the likelihood function in terms of the values for which equation 3.11 is at its maximum from the standard maximum likelihood theory and the

\footnotetext{
${ }^{10}$ Unlike previous studies, Monte Carlo studies are used in addressing the problem of model selection when the true asymmetric data generating process (DGP) is known.
} 
ordinary least squares principles (Gill 2004 pp.152). Given that the likelihood has a quadratic form in $\beta$ in the exponent it can be rewritten by expanding the quadratic expression and completing the square ${ }^{11}$ in $\beta$ to obtain ${ }^{12}$ :

$$
\begin{aligned}
(y-X \beta)^{\prime}(y-X \beta) & =(y-X \hat{\beta})^{\prime}(y-X \hat{\beta})+(\beta-\hat{\beta}) X^{\prime} X(\beta-\hat{\beta}) \\
= & v s^{2}+(\beta-\hat{\beta}) X^{\prime} X(\beta-\hat{\beta})
\end{aligned}
$$

where

$$
\begin{aligned}
& v=n-k \\
& \hat{\beta}=\left(X^{\prime} X\right)^{-1} X^{\prime} y \\
& s^{2}=(y-X \hat{\beta})^{\prime}(y-X \hat{\beta}) / v
\end{aligned}
$$

Using these results, the likelihood function (i.e. equation 3.11) can be rewritten as:

$$
p(y \mid \beta, h)=\frac{1}{(2 \pi)^{n / 2}}\left\{h^{1 / 2} \exp \left[-\frac{h}{2}(\beta-\hat{\beta})^{\prime} X^{\prime} X(\beta-\hat{\beta})\right]\right\}\left\{h^{v / 2} \exp \left[-\frac{h v}{2 s^{-2}}\right]\right\}
$$

The first term in the curly bracket denotes the kernel of a normal density and the second term depicts a gamma density (see Poirier, 1995 pp.542-543; Zellner 1971 pp.72-75; Koop, 2003 pp. 16-37 and Rossi et al 2005 pp. 21-28). The form of the likelihood has implications for elicitation of conjugate (i.e. Normal-Gamma) priors. Importantly, the form of the likelihood determines the specific conjugate prior that will ensure computational and mathematical convenience.

Intuitively, a conjugate prior distribution is one in which when combined with the likelihood yields a posterior that falls in the same family of the distribution as the prior (E.g. exponential family). Alternatively a conjugate prior may be defined as one which belongs to the same family as the posterior.

\footnotetext{
${ }^{11}$ The basic idea here is completing the square after inserting a quantity that is simultaneously subtracted and added (therefore adding zero). This can be proved by expanding the right hand side of following

equation $(y-X \beta)^{2}=\left\{(y-X \hat{\beta})-((\beta-\hat{\beta}) X\}^{2}\right.$.

${ }^{12}$ See Koop, 2003 pp. 17 or Geweke 2005 pp. 33.
} 


\subsubsection{The Prior Distribution}

In the price transmission analysis, a prior for $\beta$ and $h$ must be elicited, which is denoted by $p(\beta, h)$. The fact that we are not conditioning on the data, means that $p(\beta, h)$ is a prior density. The posterior will be denoted by $p(\beta, h \mid y)$. It proves convenient to write $p(\beta, h)=p(\beta \mid h) p(h)$ and think in terms of a prior for $\beta$ and $h$.

The form of the likelihood function of our model (3.15) above suggests that a prior distribution which has the same functional form as the likelihood function (i.e. natural conjugate prior) will involve a normal distribution for $\beta \mid h$ and a gamma distribution for $h$ in our price transmission analysis. In other words, if a prior for $\beta$ is elicited conditional on $h$ of the form

$$
\beta \mid h \sim N\left(\underline{\beta}, h^{-1} \underline{V}\right)
$$

and a prior for $h$ of the form

$h \sim G\left(\underline{s^{2}}, \underline{v}\right)$

The posterior will also have these forms (i.e. Normal-Gamma). In terms of notation, the Natural Conjugate ${ }^{13}$ Prior for $\beta$ and $h$ is denoted by:

$$
\beta, h \sim N G\left(\underline{\beta}, \underline{V}, \underline{s^{2}}, \underline{v}\right)
$$

From equation (3.18), $\underline{\beta}$ and $\underline{V}$ are the prior mean and variance of $\beta$ in the normal-gamma prior and the prior mean and degrees of freedom of $h$ are $\underline{s^{2}}$ and $\underline{v}$ respectively. What remains is to assign these hyper parameters of the prior distribution to reflect our prior information.

This research draws from the earlier works and uses the covariates to construct the prior distribution in the spirit of Zellner, 1986b (See Geweke 2005 pp.156). In essence, the prior hyper parameters can be assigned to ensure that there is minimum difference between the ordinary least square estimates and posterior parameters of the model. In practice, this research assigns prior hyper parameter values that ensure that the prior information plays very

\footnotetext{
${ }^{13}$ A Natural Conjugate prior distribution is a prior distribution which has the same functional form as the likelihood function. Basically, it is a conjugate prior with this additional property.
} 
little role in the analysis. That is, the data is allowed to speak or remain influential in the analysis. For more details on assigning priors for regression models, see Rossi et al. (2005, pp. 26) and Zellner (1971).

\subsubsection{The Posterior Inference}

The posterior is derived as the product of the likelihood of our model and the prior. This yields a posterior of the form:

$$
\beta, h \sim N G(\bar{\beta}, \bar{V}, \bar{s}, \bar{v})
$$

where

$\bar{V}=\left(\underline{V^{-1}}+X^{\prime} X\right)^{-1}$

$\bar{V}$ defines the posterior variance which is the sum of the prior variance $\underline{V}$ and data $\operatorname{variance}\left(X^{\prime} X\right)^{-1}$.

$\bar{\beta}=\bar{V}\left(\underline{V^{-1}} \underline{\beta}+X^{\prime} X \hat{\beta}\right)$

With regards to interpretation $\bar{\beta}$ is the posterior mean, which is a weighted average of the prior mean $\underline{\beta}$ and the ordinary least squares estimates $\hat{\beta}$, where the weights reflect the strength of information in the prior $\left(\underline{V}^{-1}\right)$ and the data $X^{\prime} X$.

$\bar{v}=\underline{v}+n$

$\bar{v}$ defines the prior and sample information or size.

$\bar{S}$ is defined implicitly through the posterior sum of squared errors $\left(\bar{v} \overline{s^{2}}\right)$ below.

$$
\bar{v} \overline{s^{2}}=\underline{v s^{2}}+v s^{2}(\hat{\beta}-\underline{\beta})^{\prime}\left[\underline{V}+\left(X{ }^{\prime} X\right)^{-1}\right]^{-1}+(\hat{\beta}-\underline{\beta})
$$

The posterior sum of squared errors $\left(\overline{v s^{2}}\right)$ specified in equation (3.23) is defined as the prior sum of squared errors, ordinary least squares sum of errors (OLS) and a term which measures the conflict between prior and data information. 


\subsection{The Analytical Marginal Likelihood}

Computing the marginal likelihood directly implies the Bayesian methods are being used via analytical and numerical integration. The analytical likelihood is noted as the most accurate computation of the marginal likelihood if it exists or can be derived. This is an approach implemented in a range of Bayesian econometric applications and is one of the methods developed in this research for comparison of the alternative models of asymmetric price transmission. Additionally, other methods that can be used to approximate the marginal likelihood are approximation using the Bayesian Information Criteria, Draper's Information Criteria and the computation of the marginal likelihood from a Markov Chain Monte Carlo (MCMC) output.

Specifically, the analytical methods of computing the marginal likelihood which draws heavily from Koop (2003) among others are analytically derived in subsequent discussions for the purposes of identifying the true data generating process during model comparison and selection.

\subsubsection{Analytical Expression of the Marginal Likelihood}

Unlike many other models, in the normal linear regression model with a natural conjugate prior, the integrals in the marginal likelihood can be calculated analytically. Details of these derivations can be found in Poirer (1995 pp.542-543), Zellner (1971 pp.72-75), Koop (2003 pp.38) and Geweke (2005 pp. 62-64). Koop and Potter (1998), drawing from the analytical marginal likelihood in the linear regression model, evaluate variants of autoregressive models.

\subsubsection{Derivation of the Marginal Likelihood Using Normal Gamma Prior}

Consider a common price transmission model (e.g. ECM or Houck's model) in which a linear relationship is assumed between a dependent and a given set of explanatory variables. The price transmission model can be analysed in a similar manner to the standard regression model with a natural conjugate prior. The analytical expression of the marginal likelihood is well known (see Judge, Griffths, Hill, Lutkepohl and Lee 1985 page 129). For the standard asymmetric price transmission regression with a response variable y and explanatory variables 
$X=\left(x_{1}, x_{2} \ldots x_{k}\right)$ as previously defined in equation 3.7 the following information is required to calculate the marginal likelihood.

The Normal Gamma prior for the model $\mathrm{M}$ is given by

$$
\beta, h \mid M \sim N G\left(\underline{\beta}, \underline{V}, \underline{s^{2}}, \underline{v}\right)
$$

The Sample Information:

1. The sample size $n$

2. The ordinary least squares estimates of the parameter vector $\beta_{\text {ols }}$

$$
\beta_{o l s}=\left(X^{\prime} X\right)^{-1} X^{\prime} y
$$

3. The moment matrix $X^{\prime} X$.

4. The sum of squares errors:

$$
e^{2}=\left(y-X \beta_{\text {ols }}\right)^{\prime}\left(y-X \beta_{\text {ols }}\right)
$$

Combining the sample information with the prior information, the posterior takes the form $\beta, h \mid M \sim N G(\bar{\beta}, \bar{V}, \bar{s}, \bar{v})$, defined in equations (3.7)-(3.23) and the Marginal Likelihood $^{14}$ of the model defined in equation 3.7 becomes:

$$
p(y \mid M)=\frac{\tau(\bar{v} / 2)\left(\underline{v} \underline{s}^{2}\right)^{\underline{v} / 2}|\bar{V}|^{1 / 2}\left(\bar{v} \overline{s^{2}}\right)^{-\frac{\bar{v}}{2}}}{\tau(\underline{v} / 2) \pi^{n / 2}|\underline{V}|^{1 / 2}}
$$

and $\tau()$ is defined as the natural logarithm of the absolute value of a Gamma function ${ }^{15}$ and $|\bar{V}|$ and $|\underline{V}|$ are the determinant matrices of $\bar{V}$ and $\underline{V}$ (i.e. posterior and prior variances) respectively. The integral in the marginal likelihood was solved analytically to obtain the marginal likelihood (i.e. equation 3.25) by integrating out beta and sigma (Zellner, 1971).

\footnotetext{
${ }^{14}$ Equation 3.25 denotes the marginal likelihood formula for the normal linear model with a natural conjugate prior. Koop (2003, pp. 23-25), Geweke (2005 pp. 64-65) and Zellner (1971 pp. 72-75) details the derivation of this formula.

${ }^{15}$ See Poirier (1995, p.98) for a definition of a Gamma function.
} 


\subsubsection{Bayes Factor}

Suppose we have two different theories which are represented by the statistical models, $M_{1}$ and $M_{2}$ which all seek to explain the data y. The Bayes factor $\left(B F_{12}\right)$ for comparing the two models $M_{1}$ and $M_{2}$ which is the ratio of their marginal likelihood is defined by:

$$
\begin{aligned}
& B F_{12}=\frac{p\left(y \mid M_{1}\right)}{p\left(y \mid M_{2}\right)} \\
& =\frac{\frac{\tau\left(\overline{v_{1}} / 2\right)\left(\underline{v}_{1} \underline{s}_{1}^{2}\right)^{\underline{v}_{1} / 2}\left|\overline{V_{1}}\right|^{1 / 2}\left(\overline{v_{1}} \overline{s_{1}^{2}}\right)^{-\frac{v_{1}}{2}}}{\tau\left(\underline{v}_{1} / 2\right) \pi^{n / 2}|\underline{V}|^{1 / 2}}}{\frac{\tau\left(\overline{v_{2}} / 2\right)\left(\underline{v}_{2} \underline{s}_{2}^{2}\right)^{\underline{v}_{2} / 2}\left|\overline{V_{2}}\right|^{1 / 2}\left(\overline{v_{2}} \overline{s_{2}}\right)^{-\frac{v_{2}}{2}}}{\tau\left(\underline{v}_{2} / 2\right) \pi^{n / 2}\left|\underline{V}_{2}\right|^{1 / 2}}}
\end{aligned}
$$

Specifically, the analytical marginal likelihood derived in equation 3.25 above which gives the most accurate computation of the marginal likelihood is emphasised in the empirical analysis with artificial data in a problem of identifying a true asymmetry data generating process detailed in chapter 4 . In subsequent chapters, the analytical marginal likelihood derived in equation 3.25 is denoted by Bayesian Model Selection (BMS) to distinguish it from other model selection criteria. Model rankings derived from the analytical marginal likelihood (BMS) can be compared with approximations of the marginal likelihood such as the Bayesian Information Criteria (BIC) and Drapers's Information Criteria (DIC). Fundamentally, the ability of the analytical marginal likelihood (BMS) and Information criteria to identify the true data generating process is critically examined.

It is important to note that the Bayesian analysis of threshold models can be done in a straight forward fashion since they can be written as a standard regression model conditional on a threshold parameter $(\gamma)$. In this context Koop, (2003) demonstrates that the threshold model conditional on the threshold parameter $(\gamma)$ is simply a standard regression model with a natural conjugate prior and the analytical expression of the marginal likelihood derived in equation 3.25 above is valid. Koop (2003) details the discussion on the Bayesian analysis of the threshold models. 


\subsubsection{Prior Sensitivity Analysis}

A prior sensitivity analysis can be implemented to examine the implications of alternative prior distribution on the posterior distribution and the marginal likelihood. This entails making a reasonable modification to the assumptions of the model (e.g. the prior distribution) and the posterior distribution and marginal likelihood are recomputed to examine if the choice of prior distribution has an undue impact on the results. The essence is to ensure a robust Bayesian inference.

This research proposes a prior sensitivity analysis exemplified in (Koop, 1999; Gill, 2003; and Geweke, 2005) which involves varying the hyper parameters of the prior distribution by equal proportions. Subsequently weak and strong prior variants are derived by increasing and decreasing the original prior variance by $50 \%$ or by a factor of 2 respectively.

\subsubsection{Derivation of the Marginal Likelihood Using No Prior Knowledge}

Consider a standard price transmission regression model specified in equation (3.27) with the explanatory variables in $\mathrm{X}$ defined to include variables measuring asymmetric adjustment as previously discussed in equations (3.7) and (3.8).

$$
y=X \beta+\varepsilon
$$

Where

$y$ is an $n \times 1$ vector of observations on the dependent variable.

$X$ is the $n \times k$ matrix of observations on the $\mathrm{k}$ independent variables.

$\beta$ is the $k \times 1$ vector of regression coefficients.

$\varepsilon$ is assumed to be independent and normally distributed: $\varepsilon \sim \operatorname{NID}\left(0, \sigma^{2}\right)$ so that the unknown parameters of the regression are $\left(\beta_{1, \ldots \ldots \ldots \ldots \ldots . . .}, \beta_{k}, \sigma^{2}\right)$.

Drawing from the assumption of the errors, the likelihood function for this model is given by:

$$
l\left(\beta, \sigma^{2} \mid X, y\right)=\left(2 \pi \sigma^{2}\right)^{-n / 2} \exp \left[-\frac{1}{2 \sigma^{2}}(y-X \beta)^{\prime}(y-X \beta)\right]
$$


Assuming no prior knowledge, price transmission dynamics can be developed under assumptions of uniform non-informative priors. Thus in contrast to the natural conjugate prior, $\beta$ is independent of $\sigma$. With no prior knowledge about the unknown parameters of the regression specification, we take all values of $\theta$ to be equally likely. Thus there is a uniform distribution of $\beta$. The prior on sigma is motivated by the appeal of the notion of invariance (Hepple, 2003). The uniform non-informative prior is defined as follows:

$$
\begin{aligned}
& p(\beta) \propto c \text { and } p\left(\sigma^{2}\right)=\frac{1}{\sigma} \quad \text { Where } c \text { is a constant. } \\
& p(\beta, \sigma)=p(\beta) p(\sigma) \propto \frac{1}{\sigma}
\end{aligned}
$$

The joint posterior density which is a function of the likelihood and the prior is given by $p\left(\beta, \theta, \sigma^{2} \mid y\right) . \sigma^{2}$ and $\beta$ can analytically be integrated out to give the marginal for $\theta$, $p(\theta \mid y)$. The marginal likelihood then becomes the integral of the marginal density function and is given by:

$$
p\left(y \mid M_{o l s}\right)=\tau\left(\frac{n-k}{2}\right) \cdot \frac{1}{2 \pi(n-k) / 2} \cdot \frac{1}{\left|X^{\prime} X\right|^{1 / 2}} \cdot \frac{1}{s^{2}(n-k) / 2}
$$

where $s^{2}$ is the residual sum of squares of the regression of $y$ on $X$.

An alternative price transmission model with $p$ additional $X$ variables can be defined as a basis of comparison. Empirical applications of model comparison in nested models draw from Gill (2002). Hepple (2003) considers nested as well as non-nested model comparison using the assumptions of uniform non informative priors in a recent application. 


\subsubsection{Marginal likelihood Approximation Using Bayesian Information Criteria (BIC)}

The $\mathrm{BIC}^{16}$ is a penalized measure of fit designed as an asymptotic approximation to the marginal likelihood. It employs the maximum likelihood estimates as the base of the approximation and the validity of this depends on the number of assumptions. For regular statistical models, it may be shown that as $n$ increases, the marginal likelihood may be approximated by terms from a Taylor series expansion around the maximum of the likelihood function. The details can be found in Rossi et al. (2005 pp. 163-165) and the final expression is:

$$
\log p\left(y \mid M_{1}\right)=\log p\left(y \mid \theta_{1}, M_{1}\right)-(k / 2) \log n+o\left(n^{-1 / 2}\right)
$$

where $\log p\left(y \mid \theta_{1}, M_{1}\right)$ is the log-likelihood value at the maximum. The term $-(k / 2) \log (n)$ adjusts the log-likelihood value for the parameters of the model (in relation to sample size) so that models with more parameters are not automatically favored. The term o denotes the estimation errors. This type of adjustment is also standard in other similar measures of log likelihood-based model comparison criterion such as Akaike`s Information Criterion (AIC). The idea of the AIC (Akaike, 1973, 1974) is to select the model that minimises the negative likelihood penalised by the number of parameters as specified in equation (3.32).

$$
A I C=-2 \log p(y \mid \theta)+2 p
$$

However, because the actual marginal likelihood is based on integration across all the parameter space of the model, it automatically takes the model complexity into account and does not need such an adjustment. Kass (1993) and Kass and Rafftery (1995) suggest using the (BIC) as a substitute for full calculation of the Bayes factor since the BIC can be calculated without specifying priors. The intuition is that BIC is an approximation of the marginal likelihood and subsequently it can be expressed in terms of the Bayes factor which is a ratio of the logarithm of the marginal likelihood (see Kass and Wasserman, 1992; Rafftery, 1995; Wasserman, 2000). In comparing two models with the BIC, Rafftery (1995) and Schwarz (1978) showed that twice the log of Bayes' factor $\left(B F_{12}\right)$ for comparing model 1 to model 2 given the data y is approximately equal to the difference in the BICs for the two models.

\footnotetext{
${ }^{16}$ Unlike the Akaike Information Criteria, BIC is derived within a Bayesian framework.
} 
$B I C=-2 \log p(y \mid \theta)+p \log (n)$

$\Delta B I C=B I C_{\bmod e l 1}-B I C_{\bmod e l 2}$

$\Delta B I C \approx-2 \log \left(B F_{12}\right)$

Thus an exponential of $\left(-\frac{1}{2} \Delta B I C\right)$ provides an approximation to the Bayes factor.

\subsubsection{Marginal likelihood Approximation Using Draper's Information Criteria (DIC)}

The exploration of Bayes factor estimation has culminated in numerous studies suggesting modifications to the BIC. Generally most of these modifications are typical of the modifications incorporated into Drapers's (1995) information criterion, which is given by the following equation:

$D I C=-2 \log p(y \mid \theta)+p \log (n / 2 \pi)$

In the DIC, the sample size $n$ is replaced by the term $n / 2 \pi$. Draper (1995) notes that the $2 \pi$ term typically appears in approximations to the Bayes factor but is often omitted because it is asymptotically negligible. Draper (1995) asserts that it should nonetheless be included in Bayesian information-theoretic criteria because of improvements in finite samples. Alternatively, Pauler (1998) proposes a similar criterion that incorporates the same term.

\subsection{An Overview of the Bayesian Model Selection Criteria}

Bayesian Inference on model selection uses the concept of the marginal likelihood (Gelfand and Dey, 1994) as the main model comparison tool and information Criteria as an ad hoc measure of model selection (Koop, 2003).

The intuition underpinning the concept of the marginal likelihood is that models should be evaluated on the probability they assign the data or on the probability that they generated the data. Using a graphical presentation, Figure 2 below captioned Bayesian Framework for model selection summarizes or outlines the Bayesian model selection methods or tools 


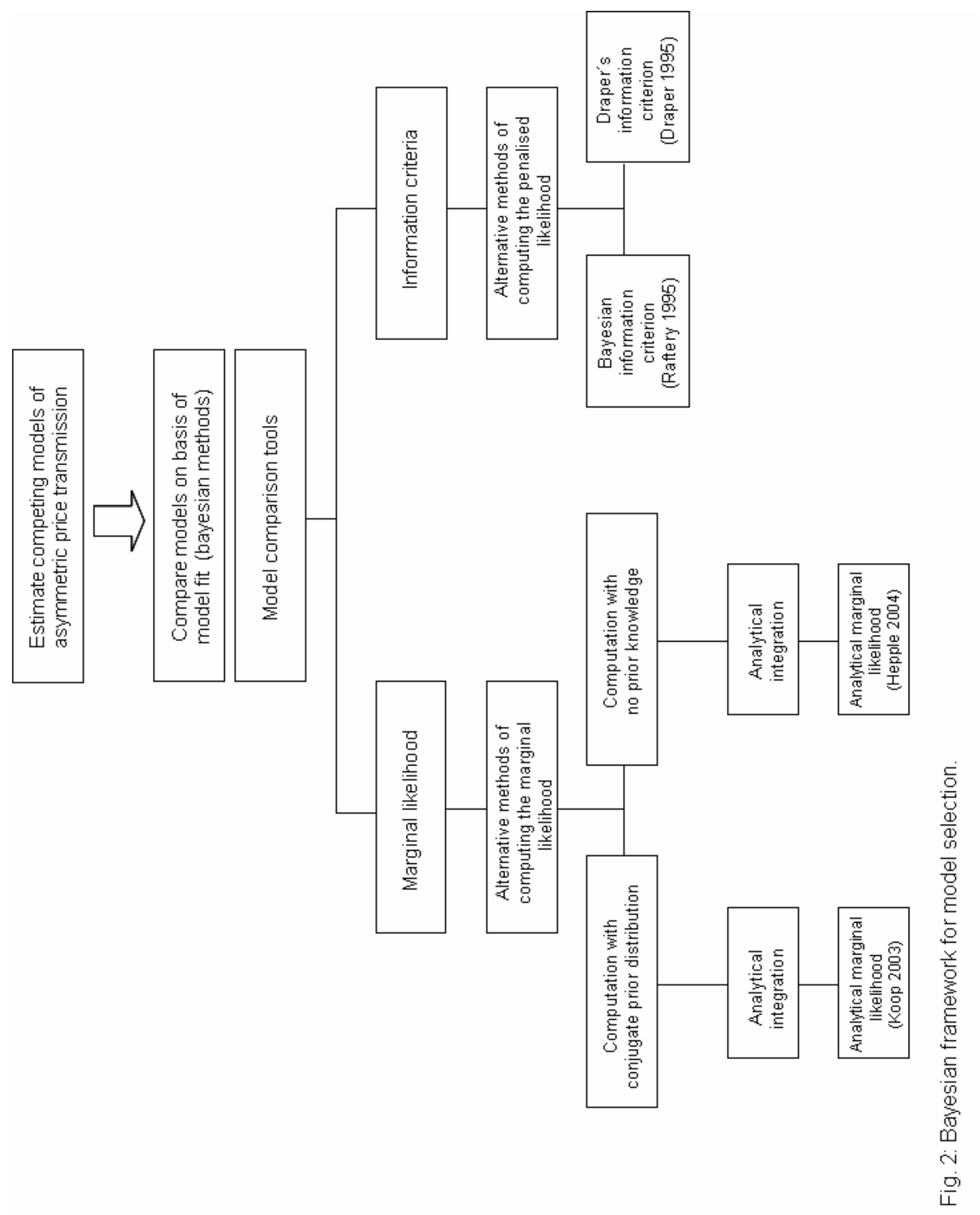


discussed in this chapter. Importantly, Figure 2 shows two main model comparison tools: the marginal likelihood and information criteria.

The marginal likelihood can be computed using prior information such as a prior distribution which has the same functional form as the likelihood (i.e. conjugate prior distribution, Koop, 2003) or alternatively the marginal likelihood can be computed with no prior knowledge (Hepple, 2003).

The Bayesian framework for model selection outlined also illustrates two information criteria for model comparison as the Draper's Information Criteria (DIC) and the Bayesian Information Criteria (BIC).

\subsection{Strength and limitations of the Bayesian Methodology}

The Bayesian Methods adopted allows for rigorous model comparison and selection with a flexible and conceptually simple framework. The nature or the number of models under examination does not affect the logic of the calculation of the marginal likelihood and its approximations in the model comparison exercise. The two main drawbacks to the Bayesian methods are the need to specify informative priors and the computational difficulty in calculating the marginal likelihood. A prior sensitivity analysis can be implemented after specification of informative priors to avoid this drawback and ensure a robust inference. The computational difficulties in calculating the marginal likelihood are minimised by implementing analytical methods for which analytical results of the posterior can be derived (see Zellner, 1971 or Koop, 2003).

\subsection{Summary and Conclusions}

Chapter three set out the Bayesian theory on model choice in the context of Bayesian Econometric Methodology. Bayesian estimation was introduced and the building blocks of a Bayesian analysis such as the prior distribution, the likelihood function and the posterior inference were discussed.

The logic of the Bayesian inference suggests that a model should be evaluated based on the probability that it generated the data or on the probability it assigns the data. Drawing from 
this intuition, the methods of choosing between alternative models are based on the marginal likelihood associated with each model. In choosing a single model, the researcher chooses the model which yields the largest marginal likelihood or alternatively ranks the models on the basis of the marginal likelihood. The marginal likelihood can be computed using analytical methods. Calculating marginal likelihoods is computationally demanding and this prompts our interest in using short cut and easy to calculate methods such as information criteria (i.e. BIC or DIC) for summarizing the data evidence in favor of a model.

Bayesian analysis of the asymmetric price transmission models was developed within the context of the regression specification. Model estimation in the context of a Bayesian analysis requires that all unknown parameters of the regression analysis are assumed to be random and assigned a prior distribution. These prior distributions are derived using the likelihood function of the regression model. Essentially, a natural conjugate prior which has the same functional form as the likelihood function is derived. In order to complete the prior elicitation, prior hyper parameters are assigned to each prior distribution drawing from Zellner (1986b). This entails using the covariates in each regression model to construct the prior distribution.

The assumptions about the errors of the standard price transmission model are used to work out the precise form of the likelihood function. In effect the construction of the likelihood function is based on the definition of multivariate normal density. Rewriting the likelihood function in terms of the ordinary least squares estimates, the likelihood function takes the form of a normal and gamma density. This form of the likelihood function suggest that a prior distribution which has the same functional form as the likelihood function will have a normal distribution for beta and a gamma distribution for the precision (i.e. a normal gamma prior). This intuition guides the choice of the normal gamma prior for our empirical analysis.

The posterior distribution summarizes information from the prior distribution and the data. It is proportional to the product of the likelihood times the prior. Inferring from the functional form of the likelihood and the prior, the posterior distribution which is a combination of the two will also have the same normal gamma functional form. Thus confirming that the prior derived is a natural conjugate prior. For this natural conjugate prior, the marginal likelihood can be computed analytically or the integrals in the marginal likelihood can be solved by analytical integration. The marginal likelihood was also derived using uniform noninformative prior. 
In summary, the methodological objective of this research is to introduce and develop a model selection criterion that can apply jointly across competing asymmetric price transmission methods or model specifications during model selection of the true underlying asymmetric data generating process. As a contribution this research introduced the Bayesian methods to the asymmetric price transmission modeling community and reviewed the Bayesian approach to model selection. In this review the Bayesian analysis of the asymmetric price transmission regression was developed within the context of a conjugate analysis (i.e. a Normal Gamma Prior distribution).Within the Bayesian framework, mathematical derivations and theoretical analysis are applied to deduce the Analytical Marginal likelihood as a rigorous model comparison and selection procedure to evaluate the competing models of asymmetric price transmission. The Bayesian Information Criteria and Draper's Information Criteria are introduced as alternative procedures for comparing the competing price transmission models. Essentially, this research has demonstrated that the Bayesian methods through the concept of the marginal likelihood and information criteria are not only conceptually simple and logical but useful for model building, criticism, comparison and selection in the models of asymmetric price transmission. The Bayesian methodology adopted offers a flexible and conceptually simple framework for rigorous comparison of any number of competing models. 


\section{A Monte Carlo Comparison of Marginal Likelihoods and Information Criteria in the Econometric Models of Asymmetric Price Transmission}

This chapter uses artificial data to demonstrate the application of the model selection methods. Subsequently, using a Bayesian analysis, different competing models of asymmetric price transmission are compared on the basis of the marginal likelihood they attain. The marginal likelihood is computed using a tractable method such as the Analytical Method (Koop, 2003). Model rankings for the alternative models are derived using the marginal likelihood as a criterion. The model rankings derived from the marginal likelihood is compared with that of the Bayesian Information Criteria, Drapers Information Criteria ${ }^{17}$ (DIC; Draper, 1995) and Akaike Information Criteria (AIC).

Essentially, the relative performance of the Bayesian model selection techniques is evaluated using Monte Carlo simulations when the true asymmetric data generating process is known. The Monte Carlo study is repeated for different sample sizes, different amount of noise in the model and various differences in the asymmetric adjustment parameters to determine how the performance of the model selection procedure (i.e. Marginal likelihood and Information Criteria) changes with changes in sample size, the amount of noise in the model and the strength of asymmetry. Fundamentally, the ability of the competing models to recover the true model is measured (i.e. True Model Recovery Rates).

The Bayesian estimation emphasises the use of a conjugate (i.e. Normal-Gamma) prior distribution. Subsequently, a prior sensitivity analysis is implemented to examine the implications of alternative prior distributions on the posterior distribution and the computed marginal likelihoods to ensure a robust Bayesian inference.

\subsection{Data Simulation Experiment}

The main objective of the simulation experiment is to find out whether the Bayesian methods are capable of identifying a true model from which an artificial data is generated (i.e. the specific asymmetric data generating process).

\footnotetext{
${ }^{17}$ The relatively new information criteria, Draper's Information Criteria shares the features of the Bayesian Information Criteria.
} 
The use of artificial data allows us to know the true data generating process in an empirical research. It can also be used to investigate the performance of the computational algorithm aside that of model properties. Fundamentally, since the values of parameters in the true model or data generating process are chosen, the results that econometric methods or model using these data should give are known. Subsequently, recent publications have addressed the issue of identifying a true data generating process using a single artificially simulated dataset in variants of econometric models. Berg, Meyer and Yu (2004) using a single simulated data generated from a stochastic volatility model that includes a jump component examined whether the Bayesian methods are capable of identifying it out of alternative stochastic volatility specifications. Similarly, Lopes and Salazar (2003) using a single simulated data from a specific logistic smooth transition model examined the ability of the Bayesian model selection criteria to identify the true model out of alternative model specifications. The emphasis of using a single artificial dataset is underpinned by the need to minimise computational burden arising from the Bayesian analysis and also the fact that the researcher knows what the results of the estimated model should be since the parameters of the model were assigned.

An alternative is to use Monte Carlo simulations and explore the analysis in repeated samples. This research designs a Monte Carlo experiment and uses 1000 simulations to examine whether the Bayesian model selection criteria on the average identify a true asymmetry data generating process out of competing alternative models. Importantly, the Monte Carlo simulations sheds light on the relative performance of the model selection procedures in a price transmission modeling framework of which no studies have been undertaken.

Data simulation of the research entails generating data from a specific asymmetric data generating process. Drawing upon the experimental designs of Cook et al.(1999, 2000, and 2003) and Holly ${ }^{18}$ et al. (2003), artificial data is generated from the Granger and Lee (1987) asymmetric error correction model and the von Cramon-Taubadel and Loy (1996) asymmetric error correction models. Using a sample size of $n=250$ observations, proceeding discussion illustrates how artificial data are generated from alternative asymmetry data generation process.

\footnotetext{
${ }^{18}$ The adopted data generating process which draws on the experimental design of Holly et al (2003) is detailed in the journal of Computational Economics.
} 
Standard Asymmetric Error Correction Model (SECM)

The standard asymmetric error correction model data generating process (DGP) can be characterised as follows:

$\Delta P_{A, t}=\beta_{o}+\beta_{1} \Delta P_{B, t}+\beta_{2}{ }^{+} E C T^{+}{ }_{t-1}+\beta_{2}{ }^{-} E C T^{-}{ }_{t-1}+\varepsilon$

$P_{A}$ and $P_{B}$ are generated as I(1) non stationary variables that are cointegrated. There exist an equilibrium relationship between $P_{A}$ and $P_{B}$ which produces $I(0)$ stationary series. This equilibrium equation is estimated by least squares and the lagged deviation from this regression is referred to as the Error Correction Term (ECT).

$P_{A, t}=\beta_{o}+\beta_{1} P_{B, t}+\varepsilon_{t}$

Where $E C T=P_{A t}-\beta_{0}-\beta_{1} P_{B t}$

The ECT is decomposed into positive and negative deviations (using a Wolffram segmentation, Granger and Lee, 1987) which are plugged into the asymmetric error correction model above (4.1). Asymmetry is introduced by allowing the speed of adjustment to differ for the positive and negative components of the Error Correction Term (ECT) since the long run relationship captured by the ECT was implicitly symmetric (see Cook et al., 2000 pp. 224; Cook et al., 2003 pp. 612 ; Holly et al., 2003 pp. 201).

Following the experimental design of Holly et al. (2003) among others, the values of $\left(\beta_{2}{ }^{+}, \beta_{2}{ }^{-}\right) \in(-0.25,-0.50) \operatorname{or}(-0.25,-0.75)$ are considered for the coefficients of the asymmetric error correction terms. $\beta_{\mathrm{o}}$ and $\beta_{1}$ are set to 3 and 0.7 respectively. Using a sample size of $\mathrm{n}, \varepsilon$ is generated as i.i.d. draws from the standard normal distribution. The asymmetric error correction model data generating process $\Delta P_{A, t}$ is constructed using the values for beta, the error correction terms, changes in $P_{B}$ and the error term through equation 4.1. Noticeably, we consider strong and subtler asymmetries in the true data generating process (DGP). 
Complex Asymmetric Error Correction Model (CECM)

Wolffram segmentation of $\Delta P_{B, t}$ leads to the following complex asymmetric error correction model data generating process (von Cramon-Taubadel and Loy, 1996):

$\Delta P_{A, t}=\beta_{\mathrm{o}}+\beta_{1}^{+} \Delta P_{B, t}^{+}+\beta_{1}^{-} \Delta P_{B, t}^{-}+\beta_{2}{ }^{+} E C T^{+}{ }_{t-1}+\beta_{2}{ }^{-} E C T^{-}{ }_{t-1}+\varepsilon$

With the values of $\left(\beta_{2}^{+}, \beta_{2}^{-}\right) \in(-0.25,-0.50) \operatorname{or}(-0.25,-0.75)$ and $\left(\beta_{1}^{+}, \beta_{1}^{-}\right) \in(0.95,0.20)$ and $\beta_{\text {o }}$ is set to 3 and $\varepsilon$ is generated as i.i.d. draws from the standard normal distribution with a sample size of $\mathrm{n}$. The remaining model variables were derived as in the previous asymmetric error correction model (i.e. 4.1). As discussed above, asymmetry is introduced by allowing the speed of adjustment to differ for the positive and negative components of the Error Correction Term (ECT) since the long run relationship captured by the ECT (i.e. cointegration relationship between the I(1) variables $P_{A}$ and $P_{B}$ ) was implicitly symmetric (See Holly et al, 2003 pp. 201). $\Delta P_{A, t}$ can be constructed using the values for beta, the positive and negative components of the error correction terms, positive and negative changes in $P_{B}$ and the error term as specified in equation 4.3 .

In summary, this study draws from the publications of Cook et al. (2000) and Holly et al. (2003) among others to derive the Granger and Lee asymmetric error correction model data generating process specified in equation 4.1 and the von Cramon-Taubadel and Loy asymmetric error correction model specified in equation 4.3 above to examine whether the Bayesian model selection technique show that the test based on the asymmetric error correction model is on the average more appropriate for these data than the test based on the Houck's model. Additionally, the effects of changes in sample size, error variance and the difference in asymmetric adjustment parameters on the model selection techniques are determined.

In addition to the complex and standard models, this study examines a simple asymmetric data generating process detailed in Wolffram (2005) and specified as follows:

$$
P_{A, t}=\beta_{o}+\beta_{1}^{+} P_{B, t}^{+}+\beta_{1}^{-} P_{B, t}^{-}+\varepsilon
$$


The Monte Carlo simulation is started by generating $\mathrm{n}=250$ observations of $P_{B}$ as independent draws from normal distribution with mean zero and a variance of one. $P_{B}$ is then split into increasing and decreasing components (i.e. $P_{B}^{+}$and $P_{B}^{-}$). Asymmetry is introduced by allowing the coefficients of $P_{B}^{+}$and $P_{B}^{-}$in equation (4.4) to differ and $\varepsilon$ is generated as i.i.d. draws from the standard normal distribution with a sample size of $\mathrm{n}$. The values of $\left(\beta_{1}{ }^{+}, \beta_{1}^{-}\right) \in(0.25,0.50) \operatorname{or}(0.25,0.75)$ are considered for the asymmetric adjustment parameters and $\beta_{\mathrm{o}}$ is set to $0.7 . P_{A, t}$ can be constructed using the values for beta, increasing and decreasing components $P_{B, t}$ (i.e. $P_{B}^{+}$and $P_{B}^{-}$) and the error term as specified in equation 4.4. Symmetric price transmission is rejected if the coefficients $\beta_{1}^{+}$and $\beta_{1}^{-}$are significantly different from one another. Wolffram (2005) provides extensions and a fundamental discussion in support of the above equation by comparing it to alternative approaches of detecting asymmetry.

Following Holly et al. (2003), preliminary investigations of this research used the Granger and Lee asymmetric ECM with the coefficients ${ }^{19}$ of the asymmetric error correction terms belonging to:

$\left(\beta_{2}{ }^{+}, \beta_{2}{ }^{-}\right) \in\{(0.25,0.75) \operatorname{or}(0.25,0.50) \operatorname{or}(-0.25,-0.75) \operatorname{or}(-0.25,-0.50)\}$

as demonstrated in the journal of computational economics and finds similar trends and patterns in the model selection analysis.

\subsection{Models and Prior Hyper Parameters}

For the purpose of demonstrating how the Bayesian methods can be used to provide a rigorous comparison of the different asymmetry test, this research focuses on common or standard models of asymmetric price transmission to facilitate implementation of the Analytical Marginal Likelihood whilst reducing the computational burden. A fundamental objective of this chapter is to compare the conventional Houck approach and the standard ECM approach when the true data generating process is known. Subsequently, using simulated data this research establishes whether the model selection criteria points to the $\mathrm{ECM}$ when the true data generating process is the ECM.

\footnotetext{
${ }^{19}$ Similarly, Cook et al (2000) generates artificial data from the Granger and Lee asymmetric ECM using the same coefficients in the Journal of Computational Economics.
} 
Specifically, a natural conjugate (e.g. normal gamma) prior is used as previously discussed in chapter 3. This involves using normal priors on all regression coefficients and a gamma prior for sigma. Assigning hyper parameters for the Normal gamma priors concludes the prior elicitation. In the context of the Natural Conjugate prior, the prior is elicited to be relatively non informative or to ensure minimal difference between posterior and least squares estimates. This could be done in several ways. One possibility is to use the covariates to construct the prior hyper parameters of the distribution. The idea is to introduce the scaling of the covariates into the prior as would seem reasonable. This research uses this idea and derives the hyper parameters of the normal-gamma prior distribution from the covariates. For the normal gamma prior, the means are set to zero and the variances are constructed using the covariates in each regression model. The idea of using the covariates matrix to construct the prior distribution for the regression coefficients draws from (Zellner, 1986b; Koop, 2003 pp.15-25 and Geweke, 2005 pp. 62-65). When the prior is relatively non informative, the prior mean is not very critical and can be set to zero (Rossi et al., 2005). The degrees of freedom of the prior precision are set to a small value relative to the actual sample size as illustrated in Koop (2003). This prior hardly influences the results with minimum differences between posterior and ordinary least squares estimates. The mathematical derivations underpinning the form of the conjugate prior used is elaborated in the theoretical analysis in chapter 3 and detailed in Koop (2003 pp. 60-62).

\subsection{Bayesian Model Assessment using the Marginal likelihood}

Model assessment involves implementing standard measures of fit to all data points to determine the extent to which the model explains the data. From a Bayesian perspective, this requires computation of the marginal likelihood, since the logic of the Bayesian inference suggests that a model should be evaluated on the probability that it generated the data or on the basis of the probability it assigns the data. In effect it defines the probability of the data conditional on a model.

However, model choice using the marginal likelihood can be influenced by the model specification such as assumptions about the priors. Subsequently, it remains imperative that model checking assesses the sensitivity of the specified model to the priors (i.e. a prior sensitivity Analysis). 
Inferences are expected to be satisfactory if the entire probability model (the prior, the likelihood and all assumptions made is a "good one" in some sense. In practice, choosing the best fitting model from the selected models of asymmetric price transmission is essential since models that fit the data poorly can lead to erroneous inferences among others.

\subsection{Empirical Results of Model Comparison}

In order to compare a set of models, this research compares their model ranking using the marginal likelihood as the criterion. The intuition is to judge the alternative models on the probability they assign the data. With choice of models being the essence of the decision problem, we choose the models with the best ranking or those which yield the largest value of the marginal likelihood. The marginal likelihood plays a central role in formal Bayesian model assessment scheme.

Subsequently, model comparison in the asymmetric price transmission models is illustrated using the exact calculation of the marginal likelihood (i.e. Analytical Marginal Likelihood) and approximations of the marginal likelihood such as BIC and DIC which provides a convenient method of comparing and ranking competing models on the basis of model fit. First, the parameters of the models are estimated in a Bayesian fashion. Second, the fit of the competing models are contrasted on the basis of the marginal likelihood. The research draws heavily from the concept of the marginal likelihood (Zellner, 1971; Aitkin ,1991; McCulloch and Rossi ,1992; Carlin and Chib ,1995; Koop and Potter ,1998; Koop and Potter ,1999; Geweke , 2005) and implements the exact computation of the marginal likelihood (Koop, 2003) and asymptotic approximations of the marginal likelihood such as Bayesian Information Criteria (Kass and Raftery ,1995) and Drapers Information Criteria ( Draper, 1995).

\subsubsection{Model Estimation Results}

The alternative models were compared using the same period or sample space to ensure adequate assessment as illustrated in Enders (2004, pp. 69-72). The estimation of the selected models considered both static and dynamic structures. Different levels of asymmetry were introduced into the simulated data. The Bayesian modeling of the ECM and the Houck's models were done in the context of a standard regression analysis. In this context, the 
Bayesian estimation of the ECM is done in two steps ${ }^{20}$ as exemplified in Congdon (2002, pp. 200-207) and LeSage (1990) among others. First, the long run relationship is estimated. Second, the resulting error correction terms are plugged into the error correction model and estimated in a Bayesian fashion.

This research examined the means and standard deviations of the posteriors of the selected asymmetric price transmission models from the Bayesian estimation. For brevity, the estimation result of the standard ECM which proves superior or outperforms the other selected asymmetric price transmission models given the simulated data generated from the standard asymmetric ECM is displayed in Table 2 below. The prior used has an expectation of zero and variance constructed from the covariates in each model using the precision ${ }^{21}$ $\underline{H}=\left(k / T \underline{\sigma^{2}}\right) X^{\prime} X$ (See Zellner, 1971 and Geweke, 2005 pp. 156 for a detailed discussion). Drawing from Koop (2003) and Birkes and Dodge (1993), the prior covariance derived from each model is inflated by a common scale factor of 3. Intuitively, the idea is to allow the data to be influential by assigning less weight to the prior. The prior hyper parameters of gamma are $\underline{v}$ set to 1 , which is a small sample relative to the data sample size and $\underline{s^{2}}$ is set $3 / \sigma^{2}$. For simplicity, $\underline{s^{2}}$ can be set $1 / \sigma^{2}$. The idea is to use some scale information of the variance of the dependent variable or the residual sum of squares from the regression as deemed reasonable.

In the proceeding discussion, the standard asymmetric error correction model, the complex asymmetric error correction model and the Houck's model in first differences are denoted by SECM, CECM and HKD respectively. Additionally, the Analytical Marginal likelihood, Draper's Information Criteria, Bayesian Information Criteria and the Akaike Information Criteria are denoted by BMS, DIC, BIC and AIC respectively. The Data Generating Process is denoted by DGP.

The results in this chapter are derived from Monte Carlo simulations and are based on average across 1000 samples using simulated data with 250 observations. The data was simulated from the standard ECM (i.e. SECM) with different levels of asymmetry as follows:

\footnotetext{
${ }^{20}$ This two-step procedure was initially proposed in LeSage (1990), and also used in Shoesmith (1992), Congdon (2002) and Felix (2003) among others.

${ }^{21}$ Zellner (1986b) was the first attempt to use the covariates to construct the prior variances. A simplification is Rossi et al 2005. An alternative to Zellner's approach is to simply use reasonably large prior variances.
} 
Standard ECM DGP (1):

$\Delta P_{A, t}=3+0.7 \Delta P_{B, t}-0.25 E C T^{+}{ }_{t-1}-0.75 E C T^{-}{ }_{t-1}+\varepsilon$

Standard ECM DGP (2):

$$
\Delta P_{A, t}=3+0.7 \Delta P_{B, t}-0.25 E C T_{t-1}^{+}-0.50 E C T_{t-1}^{-}+\varepsilon
$$

From the estimated results displayed in Table 2 below, standard ECM (i.e.SECM) which best describes the simulated data derived from equation (4.5) on the basis of the marginal likelihood and information criteria tends to provides accurate estimates of the parameters of the artificial data.

From the estimated results it is evident that the difference between the least square estimates and the posterior estimates are minimal. This minimum difference suggests that the data is influential in the results or analysis. The Houck's model in sum difference provides the poorest estimation and fit to the simulated data.

${ }^{22}$ Table 2: Standard ECM DGP (1): Natural Conjugate Prior, Least Squares and Posterior Moments of the SECM (Analytical Method)

\begin{tabular}{|c|c|c|c|c|c|c|}
\hline & \multicolumn{2}{|c|}{ Prior } & \multicolumn{2}{|r|}{ Squares } & \multicolumn{2}{|c|}{ Posterior } \\
\hline Variable & Mean & Std. Error & Mean & Std. Error & Mean & Std. Error \\
\hline Intercept & 0 & 1.919 & 2.997 & 0.107 & 2.987 & 0.107 \\
\hline$\Delta P_{w, t}$ & 0 & 1.159 & 0.702 & 0.064 & 0.700 & 0.064 \\
\hline$E C T_{t-1}^{+}$ & 0 & 2.264 & -0.250 & 0.126 & -0.261 & 0.126 \\
\hline$E C T^{-}{ }_{t-1}$ & 0 & 2.248 & -0.749 & 0.125 & -0.739 & 0.125 \\
\hline
\end{tabular}

Based on 1000 Replications

Similarly, minimal differences are observed between the posterior moments and the least squares estimates for the standard ECM model when more subtle levels of asymmetry are introduced as exemplified in Table 3.

\footnotetext{
${ }^{22}$ Tables displayed in this Chapter were derived from the authors own computations using simulated data.
} 
Table 3: Standard ECM DGP (2): Natural Conjugate Prior, Least Squares and Posterior Moments of the SECM (Analytical Method)

\begin{tabular}{|c|c|c|c|c|c|c|}
\hline & \multicolumn{2}{|c|}{ Prior } & \multicolumn{2}{|r|}{ squares } & \multicolumn{2}{|c|}{ Posterior } \\
\hline Variable & Mean & Std. Error & Mean & Std. Error & Mean & Std. Error \\
\hline Intercept & 0 & 1.847 & 3.004 & 0.107 & 2.993 & 0.107 \\
\hline$\Delta P_{w, t}$ & 0 & 1.116 & 0.699 & 0.065 & 0.697 & 0.065 \\
\hline$E C T_{t-1}^{+}$ & 0 & 2.167 & -0.249 & 0.126 & -0.258 & 0.126 \\
\hline$E C T_{t-1}^{-}$ & 0 & 2.173 & -0.503 & 0.126 & -0.492 & 0.126 \\
\hline
\end{tabular}

Based on 1000 Replications

The difference in the asymmetric adjustment parameters was decreased from 0.50 to 025 . That is, the positive and negative asymmetric adjustment parameters change from 0.25 and 0.75 in table 2 above to 0.25 and 0.50 as specified in equation 4.6 and displayed in table 3 above. Thus for both strong and subtle levels of asymmetry, the differences between the posterior estimates and the least squares estimations were minimal. The results in Table 3 above were derived under a setting where the data was used to construct the prior hyper parameters.

Although this research emphasises the idea of using the data to construct the prior distribution, the traditional Bayesian approach in which the hyper parameters are assigned before seeing the data or generating the data also provides similar results in our preliminary investigations not reported here for the sake of brevity.

Noticeably, the estimation results of SECM which provides the best fitting model on the basis of the marginal likelihood and information criteria tends to provide accurate estimates of the parameters of the simulated data as illustrated in equation (4.6). It is important to note that the discussion so far has emphasised the use of moderate sample sizes of 250 and we note that using relatively large samples increased the amount of data information and improves the estimation results. 
4.4.2 Evaluation of Model Performance using Rankings derived from the Analytical Marginal Likelihood with a Natural Conjugate Prior

First, this section focuses on the most accurate computation of the Marginal Likelihood, the analytical method and draws principally from Geweke (2005, pp.62-65), Koop (2003, pp.1531), Poirier (1995 pp.542-543) or Zellner (1971, pp.72-75, 1986b). The analytical method explained in detailed in chapter 3 and the formula for computation of the analytical marginal likelihood defined in Zellner (1971) and Koop (2003) and specified in equation (3.25) was used in the construction of the figures in Table 4 below.

For each of the asymmetric price transmission models, Table 4 below reports an estimate of the logarithm of the marginal likelihood. In choosing a single model, this study chooses the one which yields the largest value of the marginal likelihood or provides model rankings using the marginal likelihood as illustrated below. For each of the model selection methods the values provided are the arithmetic mean based on 1000 samples using Monte Carlo simulations.

From the comparison of the models in Table 4 below, this research finds differences in the marginal likelihood of the selected alternative models which forms the basis for their different rankings provided.

Table 4: Standard ECM DGP (1): Analytical Marginal Likelihood (BMS), BIC, DIC and AIC of selected models

\begin{tabular}{|l|l|l|l|l|l|l|l|l|}
\hline & \multicolumn{2}{|l|}{ BMS (Koop, 2003) } & \multicolumn{2}{|c|}{ BIC } & \multicolumn{2}{c|}{ DIC } & \multicolumn{2}{c|}{ AIC } \\
\hline Model & Value & Ranking & Value & Ranking & Value & Ranking & Value & Ranking \\
\hline HKD(AR1) & -384.98 & 4 & 765.72 & 4 & 768.43 & 4 & 744.83 & 4 \\
\hline HKD & -380.79 & 3 & 756.80 & 3 & 758.61 & 3 & 742.88 & 3 \\
\hline CECM & -356.34 & 2 & 707.49 & 2 & 710.21 & 2 & 686.62 & 2 \\
\hline & & & & & & & & \\
SECM(DGP) & -354.47 & 1 & 703.01 & 1 & 705.27 & 1 & 685.60 & 1 \\
\hline
\end{tabular}

Based on 1000 Replications

The model which fits the cointegrated simulated data best according to the analytical marginal likelihood is the standard ECM, followed by the complex asymmetric ECM, static and 
dynamic Houck's model in first differences respectively. This observation is consistent with the fact that the true asymmetric data generating process is the standard error correction model (SECM). The standard Houck's model in first difference fits the data poorly as suggested by the lowest value of its marginal likelihood of -380.79 . Whilst introducing lags into the Houck's model in first difference to derive the dynamic variant worsens the fit with a marginal likelihood of -384.98. The poor performance of the Houck's model in first difference may be partly due to the omission of the cointegrating relationship which is originally incorporated into the true asymmetric ECM data generating process via the error correction mechanism. Subsequently, when the standard and complex asymmetric error correction models which incorporate the equilibrium relationships are compared with the Houck's model in first differences, a better model fit is achieved. Fundamentally, the rankings provided by the marginal likelihood underpin the importance of incorporating error correction mechanism in modeling the cointegrated data during an asymmetric price transmission analysis. Given the cointegrated data, none of the models incorporating the long-run relationship produced the poorest fit to the data. These confirm the assertions of Granger and Engle (1987) that in the presence of cointegration of the data an error correction mechanism exists.

The Houck's model in sum difference provides the poorest fit to the data with a marginal likelihood of -883 and -899 for the static and dynamic variants respectively. However, Houck's model in summed differences does not fully consider the time series property of the data used. Von Cramon-Taubadel (1998) provides a detail discussion and a fundamental clarification. Specifically, it is not compatible with cointegration and the long term information between the time series. Essentially, the discussion points to the fact that it is important that model selection takes into consideration the estimation techniques of the models being compared (i.e. pre cointegration or cointegration) and the data characteristics.

The models are also compared according to their estimation techniques such as precointegration or cointegration. Within the pre-cointegration setting the Houck's model in first difference out performs the Houck's models in sum difference given the data from the standard asymmetric ECM. In the cointegration setting the standard ECM produces a better model fit than the asymmetric error correction model with complex dynamics given the standard asymmetric ECM data generating process. Examination of the residuals of the estimated alternative models indicates a reasonable degree of normality. Additionally, Table 
4 provides model ranking on the basis of marginal likelihood approximations such as the Bayesian Information Criteria (BIC). The BIC and the Marginal Likelihood are asymptotically equivalent. The BIC is derived within a Bayesian framework. Model selection entails choosing the model which has the lowest or minimum values of the BIC. The BIC values were computed for the different lags in order to choose the appropriate model. On the basis of the BIC values provided above, it is evident that the best model given the data is the standard ECM, followed by the complex asymmetric error correction model, the static and dynamic Houck's model in first differences respectively. The static and dynamic Houck's model in summed differences provided the poorest fit to the simulated data with a BIC of 1716 and 1721 respectively. Notably, for the selected models of asymmetric price transmission, the crude approximation of the marginal likelihood (i.e. BIC) provides the same model ranking as the analytical marginal likelihood.

Model rankings are also derived from an alternative approximation of the marginal likelihood, Drapers Information Criteria (DIC, Draper, 1995) which shares the features of the Bayesian Information Criteria. Model selection involves choosing the model which has the lowest or minimum values of the DIC. The model rankings of the DIC are similar to the Analytical Marginal Likelihood and the Bayesian Information criteria. On the basis of the DIC values provided above, it is evident that the best fitting model given the data is the standard ECM, followed by the complex asymmetric error correction model and the variants of the Houck's model in first differences. Again, the static and dynamic Houck's model in summed differences provided the poorest fit to the simulated data with a DIC of 1718 and 1723 respectively.

The rankings derived by the Bayesian Information Criteria and the Analytical Marginal Likelihood are compared to the model rankings of the Akaike Information Criteria. Noticeably, the model rankings provided by the AIC and the BIC or DIC are consistent with marginal likelihood. Model rankings derived from the AIC, BIC, DIC and the Marginal likelihoods are the same. However, it is important to note that fundamental differences ${ }^{23}$ exist between AIC and BIC, DIC or the marginal likelihood. BIC, DIC and the marginal likelihoods are derived within a Bayesian framework and intended to identify the true data generating process or find the most probable model given the data. AIC is not intended to

\footnotetext{
${ }^{23}$ Zucchinni (2000) provides a fundamental discussion and clarification of the difference between the AIC and BIC. Alternatively, see Wasserman (2000).
} 
identify the true model exactly but to propose a good approximation of it or to choose the model that is closest to the true model. Alternatively, AIC aims to estimate how well each model with parameters estimated from observed data is expected to predict a new data set of future observations. Although the AIC and the Bayesian methods (i.e. BIC, DIC, Marginal Likelihood) are based on different theoretical motivations and objectives, in a broader sense they all have the same aim of identifying good models even if they differ in their exact definition of a good model.

The performance of the model selection criteria are examined using more subtle levels of asymmetry as specified in equation 4.6 and displayed in Table 5 below. The Analytical Marginal likelihood, BIC, DIC and the AIC all correctly identifies the true data generating process when the difference in the asymmetric adjustment parameters decreased from 0.50 to 0.25. Noticeably, both the AIC, BIC, DIC and the Marginal Likelihood provide the same model ranking.

Table 5: Standard ECM DGP (2): Analytical Marginal Likelihood (BMS), BIC, DIC and AIC of Selected models

\begin{tabular}{|l|l|l|l|l|l|l|l|l|}
\hline & \multicolumn{2}{|}{ BMS (Koop,2003) } & \multicolumn{2}{|c|}{ BIC } & \multicolumn{2}{c|}{ DIC } & \multicolumn{2}{c|}{ AIC } \\
\hline Model & Value & Ranking & Value & Ranking & Value & Ranking & Value & Ranking \\
\hline HKD(AR1) & -372.69 & 4 & 741.03 & 4 & 743.73 & 4 & 720.14 & 4 \\
\hline HKD & -368.54 & 3 & 732.07 & 3 & 733.87 & 3 & 718.14 & 3 \\
\hline CECM & -356.55 & 2 & 708.14 & 2 & 710.85 & 2 & 687.26 & 2 \\
\hline SECM(DGP) & -354.78 & 1 & 703.69 & 1 & 705.95 & 1 & 686.29 & 1 \\
\hline
\end{tabular}

Based on 1000 Replications

The discussion of the computations of the marginal likelihood has implemented Analytical computations using informative prior (i.e. Natural Conjugate priors or Normal Gamma) which provides the most accurate value of the marginal likelihood as emphasised by proponents ( Zellner, 1971; Koop, 2003; Geweke, 2005). Under this approach, the Bayes' factor can be easily specified. 
Importantly, all the model selection algorithms examined clearly identify the correct model out of alternative competing models or on the average points to the true asymmetric data generating process. Our estimation results with all simulated data are accurate for the true model. The results derived from 1000 Monte Carlo simulations in Tables 4 and 5 therefore indicate that information criteria and the marginal likelihood provides a holistic and consistent approach to ranking and selecting among the competing models of asymmetric price transmission. This is better explained by the ability of the alternative Bayesian criteria to select the same true model or provide the same model rankings and the fact that their identified true model is actually the true asymmetric data generating process. Using 1000 Monte Carlo simulations, this research has demonstrated that the Bayesian criteria do on average point to the appropriate asymmetry tests in repeated samples. Intuitively, practitioners modeling asymmetry without knowing the true data generating process could emphasise the Bayesian criteria to distinguish between competing models knowing from this study that the Bayesian criteria do on the average point to the true asymmetric price transmission model.

An important feature of our results is that they are generally consistent with previous studies focusing on identification of a true data generating process. In a financial market analysis, Berg, Meyer and Yu (2004) using a single simulated data generated from a specific stochastic volatility model establish that the Bayesian methods do point to the true model. Similarly, Myung (2000) in a cognitive psychology modeling framework notes that the Bayesian methods clearly identifies the true data generating process.

\subsection{Prior Sensitivity Analysis of the Analytical Marginal Likelihood}

Generally, a prior sensitivity ${ }^{24}$ analysis aims to examine the implication of the alternative prior distribution on the posterior distribution and the computed marginal likelihood. This study examined the sensitivity of the posterior means to changes in the prior distribution. This entailed re- estimating the selected models with different priors (i.e. a weak and strong variant of the original prior used). The weak and strong priors were derived by varying the variance parameter by a factor of 2 in both directions. The idea is to vary the original prior by equal proportions in both directions. Subsequently, the effect of alternative prior distributions on the

\footnotetext{
${ }^{24}$ The sensitivity of the posterior distribution to the prior distribution can be studied in a number of ways: One of the simplest ways is to vary the hyper parameters of the prior distribution by equal proportions (Geweke 2005).
} 
computed marginal likelihoods is also examined. The essence of the prior sensitivity analysis is to ensure a robust Bayesian inference.

For the sake of brevity, the prior sensitivity analysis of the SECM which outperforms the other competing models is conducted and displayed in Table 6 below. The Bayesian result with the original prior used for this model is compared to the classical results as well as the results derived using weak and strong prior variants. The minimal differences in the results of the different estimations are illustrated below in Table 6. The comparison illustrated above indicates that the original prior used in our analysis yields results that is close or shows minimal difference from the least squares estimates and the estimations using a weak and strong prior variants respectively.

Table 6: Standard ECM DGP (1): Sensitivity of the Analytical Marginal Likelihood to the Prior (SECM)

\begin{tabular}{|l|l|l|l|l|l|}
\hline \multirow{2}{*}{ Coefficient } & \multirow{2}{*}{$\begin{array}{l}\text { Least } \\
\text { Squares }\end{array}$} & \multicolumn{4}{|c|}{ Posterior Means with Prior } \\
\cline { 2 - 6 } & Weak & Original & Strong & Prior Mean \\
\hline Intercept & 2.997 & 2.992 & 2.987 & 2.977 & 0 \\
\hline$\Delta P_{w, t}$ & 0.702 & 0.701 & 0.700 & 0.698 & 0 \\
\hline$E C T_{t-1}^{+}$ & -0.250 & -0.258 & -0.261 & -0.269 & 0 \\
\hline$E C T_{t-1}^{-}$ & -0.749 & -0.744 & -0.739 & -0.730 & 0 \\
\hline & & & & & \\
\hline $\begin{array}{l}\text { Marginal } \\
\text { Likelihood }\end{array}$ & & -355.11 & -354.47 & -354.38 & \\
\hline Based on & & & & & \\
\hline
\end{tabular}

Based on 1000 Replications

Intuitively, this implies that our estimated models are insensitive to modest modifications of the prior specifications. Else, it would have shown dramatic changes in the posterior estimates or summaries. More importantly, the marginal likelihood is robust to changes in the prior distribution. The marginal likelihood of the weak and strong prior variants for the standard ECM model is -355.11 and -354.38 respectively. These show minimal difference from the marginal likelihood of -354.47 obtained using the original prior. Additionally, the ranking of the models considered is preserved when the strong and weak prior variants are used. The prior sensitivity analysis implemented in this study draws from Koop (1999), Gill (2003, pp.167-168) and Geweke (2005, pp.157) among others. 
Table 7: Standard ECM DGP (2): Sensitivity of the Analytical Marginal Likelihood to the Prior (SECM)

\begin{tabular}{|c|c|c|c|c|c|}
\hline \multirow[t]{2}{*}{ Coefficient } & \multirow{2}{*}{$\begin{array}{l}\text { Least } \\
\text { Squares }\end{array}$} & \multicolumn{4}{|c|}{ Posterior Means with Prior } \\
\hline & & Weak & Original & Strong & Prior Mean \\
\hline Intercept & 3.004 & 2.998 & 2.993 & 2.982 & 0 \\
\hline$\Delta P_{w, t}$ & 0.699 & 0.698 & 0.697 & 0.695 & 0 \\
\hline$E C T_{t-1}^{+}$ & -0.249 & -0.253 & -0.258 & -0.266 & 0 \\
\hline$E C T_{t-1}^{-}$ & -0.503 & -0.498 & -0.492 & -0.484 & 0 \\
\hline $\begin{array}{l}\text { Marginal } \\
\text { Likelihood }\end{array}$ & & -355.37 & -354.78 & -354.66 & \\
\hline
\end{tabular}

Based on 1000 Replications

Similarly, minimal differences in the results of the different estimations are illustrated above in Table 7. The comparison illustrated above indicates that the original prior used in our analysis yields results that is close or shows minimal difference from the least squares estimates and the estimations using a weak and strong prior variants respectively. In effect, the data is still influential in the analysis when more subtle levels of asymmetry are applied as specified in equation (4.6). In addition the marginal likelihood remains robust to changes in prior distribution and the model rankings are preserved when alternative priors are used.

\subsection{Analysing the Model Recovery Rates of the Tests for Asymmetry}

This section evaluates the relative performance of the model selection criteria in recovering the true data generating process by simulating the effect of sample size, the amount of noise in the model (stochastic variance) and size of the difference between the asymmetric adjustment parameters on model selection. Subsequently, the competing models are fitted to the simulated data and their ability to recover the true model measured (i.e. Model Recovery Rates). The model recovery rates define the percentage of samples in which each competing model provides a better model fit than the other competing models. In this study, all recovery rates are derived using 1000 Monte Carlo simulations. Impliedly, the amount of samples in which each model fits better than the other competing models is measured out of the 1000 samples and expressed as a percentage. In this context, the values derived from each model by selection methods are derived as the arithmetic mean based on 1000 samples. 
It is important to recognise that the concept of model recovery rates emphasised in the context of identification of a true model has been used extensively in various applications by some experts (Bozdogan, 1987; Myung, 2000; Myung et al., 2001; Gheissari and Bab-Hadiasher, 2003) among others. Alternatively, Markon and Krueger (2004) use simply the ratio defining the proportion of simulated datasets in which the correct model was selected (i.e. the selection power). Some authors including (Yang 2003) use model recovery frequencies instead of model recovery rates.

\subsubsection{Monte Carlo Simulation of the Effects of Sample Size on Model Selection}

In order to simulate the effects of sample size on model selection this study considers three sample sizes ranging from small to large corresponding to 50,150 and 500. Using an error size of 1, data is generated from equation (4.5). The results of the Monte Carlo simulations comparing the performance of the model selection methods are displayed below in Table 8 .

Generally, inspection of the recovery rates for the different model selection criteria illustrates the extent to which the true model (SECM) is recovered by each selection criteria across the different sample sizes. In the small sample size of 50, the true model was recovered at least 78 percent across the Bayesian Criteria in the top part of the Table 8. At a sample size of 500, at least 98.4 percent of the true model was recovered by the Bayesian criteria. For the sake of brevity, the Houck's model in sum difference which was included in all simulations but performed poorly on the basis of all model selection methods( BMS, BIC,DIC and AIC) and was never recovered due to poor model fit is excluded in the Table 8 above.

In comparison with the small sample recovery rates, model recoveries of the true model improve significantly when the sample size was large. Despite differences in performance among the model selection criteria, trends holding across the different criteria were evident in the simulation results. In effect, the performance of the model selection methods to select the true model (i.e. recovery rates of SECM) generally increased with increases in sample size from 50 to 500 .

The graph below shows the percentage of the simulated data in which the correct model (i.e. SECM) was selected or recovered by the model selection criteria across different sample 
sizes. Comparison of the different selection methods in the Figure 3 shows a general pattern in which recovery rates increased with increase sample sizes.

Table 8: Relative Performance of Model Selection Methods across Sample Size

\begin{tabular}{|c|c|c|c|c|}
\hline $\begin{array}{l}\text { Sample } \\
\text { Size }\end{array}$ & \multicolumn{4}{|c|}{ Model Fitted } \\
\hline \multirow[t]{6}{*}{50} & & CECM & HKD & SECM(DGP) \\
\hline & Methods & & & \\
\hline & AIC & $119(16.8 \%)$ & $127(5.6 \%)$ & $118(77.6 \%)$ \\
\hline & $\mathrm{BIC}$ & $129(5.9 \%)$ & $134(12.7 \%)$ & $127(81.4 \%)$ \\
\hline & DIC & $132(3.7 \%)$ & $135(15 \%)$ & $129(81.3 \%)$ \\
\hline & BMS & $-68(6.3 \%)$ & $-69(15.7 \%)$ & $-67 \quad(78 \%)$ \\
\hline \multirow{6}{*}{150} & & $a^{\prime}$ & , & cragrach \\
\hline & Methods & & & \\
\hline & $\mathrm{AIC}$ & $403(16.2 \%)$ & $434(0 \%)$ & $402(83.8 \%)$ \\
\hline & $\mathrm{BIC}$ & $420(2.9 \%)$ & $446(0.1 \%)$ & $416(97 \%)$ \\
\hline & DIC & $423(2.5 \%)$ & $448(0.1 \%)$ & $419(97.4 \%)$ \\
\hline & BMS & $-213(4.1 \%)$ & $-226(0.1 \%)$ & $-211(95.8 \%)$ \\
\hline \multirow[t]{6}{*}{500} & & CECM & HKD & SECM(DGP) \\
\hline & Methods & & & \\
\hline & AIC & $1396(15.2 \%)$ & $1512(0 \%)$ & $1395(84.8 \%)$ \\
\hline & $\mathrm{BIC}$ & $1422(0.9 \%)$ & $1528(0 \%)$ & $1416(99.1 \%)$ \\
\hline & DIC & $1424(0.5 \%)$ & $1530(0 \%)$ & $1419(99.5 \%)$ \\
\hline & BMS & $-713(1.6 \%)$ & $-766(0 \%)$ & $-711(98.4 \%)$ \\
\hline
\end{tabular}

Based on 1000 Replications

However, two distinct patterns can also be identified in the graph below. First, the Bayesian Criteria (BMS, DIC, and BIC) performed similarly to one another and their recovery rates varied strongly as a function of sample size. Second, although AIC performed well in the small samples, it did not make substantial gains in recovery rates as the sample size increased.

The observed patterns are consistent with previous studies on model selection. Ichikawa (1998)'s simulation results in a factor analysis indicated that the ability of AIC to select a true model rapidly increased with sample size but at larger sample sizes it continued to exhibit a slight tendency to select complex models.

Similarly, Markon and Krueger (2004) reviewed existing work on factor analysis and noted that AIC performs relatively well in small samples, but is inconsistent and does not improve 
in performance in large samples whilst BIC in contrast appears to perform relatively poorly in small samples, but is consistent and improves in performance with sample size. Fishler et al. (2002) also investigated the performance of BIC in a factor analysis and their results suggest that BIC performs poorly at small sample sizes, but improves with increasing sample size to eventually choose the correct model with perfect probability.

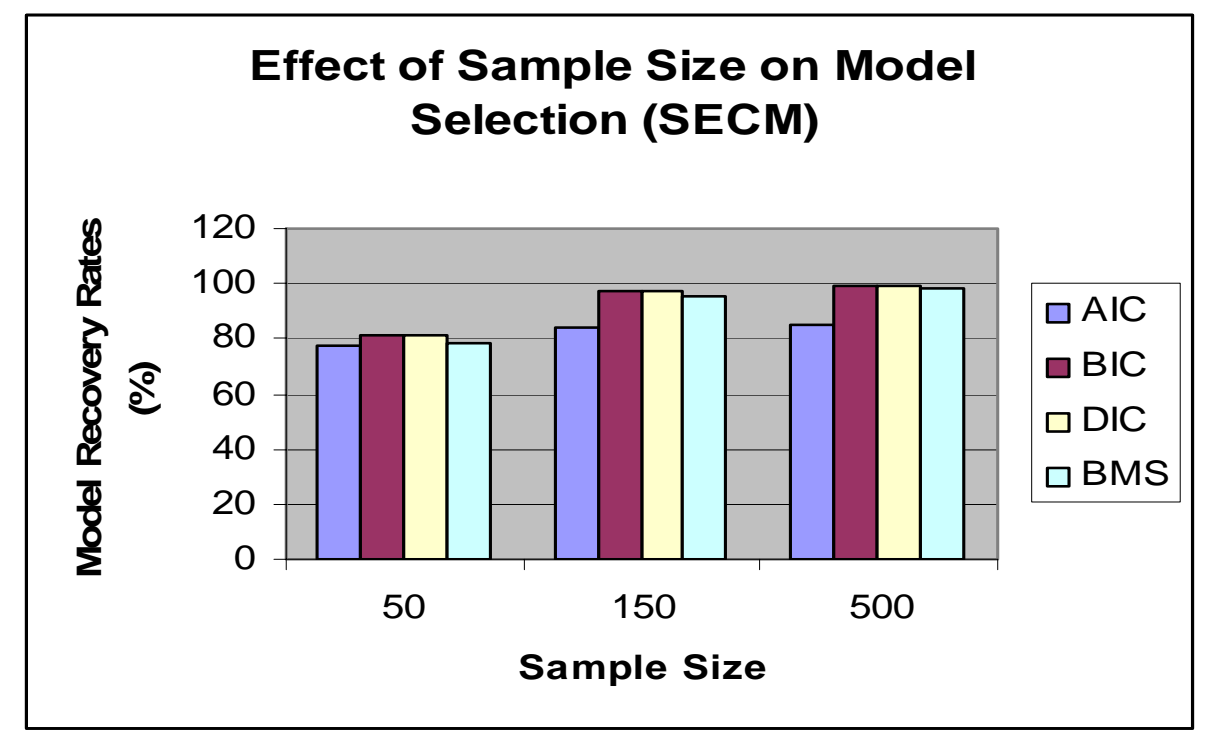

Figure 3: Effects of Sample Size on Model Selection

Although BIC, DIC and BMS performed similarly there was a slight tendency for DIC to out perform BIC with the highest recovery rates of 97.4 and 99.5 percent in both moderate and large samples sizes. Similarly, Markon and Krueger (2004) noted that the DIC outperforms the BIC in a structural equation modeling framework. The tendencies of DIC to out perform BIC in the asymmetric price transmission modeling framework are better explained by the fact that the improved performance of the DIC was the motivation for its development and implementation in Draper (1995). Additionally, the DIC outperforms the BMS slightly.

In summary, the model selection methods performed reasonably well in identifying the true model, though their ability to recover the true asymmetric data generating process increases with increase in sample size as illustrated in the figure 3. Intuitively, the results points to the fact that the sample sizes are important in the selection of the true asymmetric data generating process during price transmission analysis. Generally, larger sample sizes might improve the ability of the model selection methods to make correct inferences about asymmetric price transmission models. 
The relatively new information criteria, Draper's Information Criteria (DIC, Draper, 1995), which shares the features of the Bayesian Information Criteria, performs similarly to or better than the BIC in asymmetric price transmission modeling framework.

An important feature of our results is that they generally echo existing theoretical and empirical work on the performance of model selection methods in other applications. For instance investigations with generalized linear models have demonstrated that BIC is consistent, that is it tends to choose the true model with probability equal to one in large samples but performs poorly in small samples (Hurvich and Tsai, 1990; Bickel and Zhang, 1992). AIC in contrast is not consistent but performs relatively well in small samples.

\subsubsection{Monte Carlo Simulation of the Effects of Stochastic Variance on Model Selection}

In order to simulate the effects of noise level on model selection this study considers three different standard deviations ranging relatively from small to large and corresponding to 1.0, 2.0 and 3.0. Using a sample size of 150, data is generated from equation (4.5) with the different error sizes. Essentially, the data fitting abilities of alternative models are compared in relation to the true model as the error in the data generating process was increased systematically. The results of 1000 Monte Carlo simulations comparing the performance of the model selection methods as error size $(\sigma)$ increased are displayed in Table 9 below.

Generally, as expected, recovery rates of the true asymmetric data generating process (i.e. SECM) declined for all model selection methods as the error increased. With a moderate sample size of 150 and an error size $(\sigma)$ of 3 the Houck's model in first difference fits the data better on the basis of the Bayesian criteria (i.e. BIC, DIC and BMS) although the standard asymmetric ECM is the true data generating process. Using the DIC and BMS, the Houck's model in first difference was recovered 59.7 percent and 64.3 percent respectively as indicated in the top part of Table 9 below.

The improvement in fit and the high model recovery of the Houck's model in first difference over the SECM or the true data generating process may be partly due to the model's flexibility to capture random error and not because it accurately approximates the model that generated the data. It is imperative to note that as error decreases the Houck's model fits 
poorly as indicated in the bottom part of Table 9 with recovery rates ranging between 0 0.1 percent for the model selection methods.

Table 9: Relative Performance of Model Selection Methods across Noise levels

\begin{tabular}{|c|c|c|c|c|}
\hline $\begin{array}{c}\text { Error } \\
\text { Size }\end{array}$ & & Model Fi & & \\
\hline \multirow[t]{6}{*}{3} & & CECM & HKD & SECM(DGP) \\
\hline & Methods & & & \\
\hline & $\mathrm{AIC}$ & 711 (10.1\%) & 713 (25.1\%) & 709 (64.8\%) \\
\hline & $\mathrm{BIC}$ & $728(0.4 \%)$ & $725(55.5 \%)$ & $724(44.1 \%)$ \\
\hline & DIC & $731(0.3 \%)$ & $726(59.7 \%)$ & $726(40 \%)$ \\
\hline & BMS & $-371(0.1 \%)$ & $-367(64.3 \%)$ & $-368(35.6 \%)$ \\
\hline \multirow{7}{*}{2} & & & & \\
\hline & & CECM & HKD & SECM(DGP) \\
\hline & Methods & & & \\
\hline & AIC & $596(13.4 \%)$ & $604(5.8 \%)$ & $595(80.8 \%)$ \\
\hline & $\mathrm{BIC}$ & $614(1.9 \%)$ & $615(20 \%)$ & $610(78.1 \%)$ \\
\hline & $\mathrm{DIC}$ & $616(1.5 \%)$ & $617(23.4 \%)$ & $612(75.1 \%)$ \\
\hline & BMS & $-311(1.4 \%)$ & $-311(23.3 \%)$ & $-309(75.3 \%)$ \\
\hline \multirow[t]{6}{*}{1} & & CECM & HKD & SECM(DGP) \\
\hline & Methods & & & \\
\hline & AIC & $402(17 \%)$ & $434(0 \%)$ & $401(83 \%)$ \\
\hline & $\mathrm{BIC}$ & $420(3.4 \%)$ & $446(0.1 \%)$ & $416(96.5 \%)$ \\
\hline & $\mathrm{DIC}$ & $423 \quad(3 \%)$ & $447(0.1 \%)$ & 418 (96.9\%) \\
\hline & BMS & $-212 \quad(4.9 \%)$ & $-225(0.1 \%)$ & $-211(95 \%)$ \\
\hline
\end{tabular}

Based on 1000 Replications

Intuitively, higher noise levels make it difficult for the model selection methods to identify the true asymmetric model or alternatively the performance of the model selection methods deteriorates with high levels of noise in the asymmetric price transmission modeling framework.

These results are generally consistent with those obtained by experts who studied the effects of noise levels on model selection in other applications such as linear regression models and computer vision applications (See Myung, 2000; Gheissari and Bab-Hadiashar, 2003; Yang, 2003). Importantly, Yang (2003) finds that the recovery rates of the true data generating process decreases with increasing noise levels in linear regression models. In conclusion Yang notes that selection can yield the wrong model at higher noise levels. Alternatively, without regards to model selection methods (i.e. Marginal Likelihood and Information Criteria), Cook et al.( 1999) using a mathematical derivation and Monte Carlo experiment demonstrates that 
the test for asymmetry in particular depends on the sample size and the stochastic variance of the true data generating process in recent publications.

Figure 4 shows the percentage of the simulated data in which the correct model (i.e. SECM) was selected or recovered among competing models by the model selection criteria as the amount of noise in the data generating process increased. Comparison of the different selection methods in Figure 4 shows a general trend in which recovery rates decreased with increasing error sizes. In effect, the performance of all model selection algorithms analysed deteriorates with increasing amount of noise in the true asymmetric price transmission data generating process.

Generally, two distinct trends can also be identified in the graph. First, the Bayesian Criteria (BMS, DIC, and BIC) performed similarly to one another with their recovery rates decreasing substantially as noise levels increased.

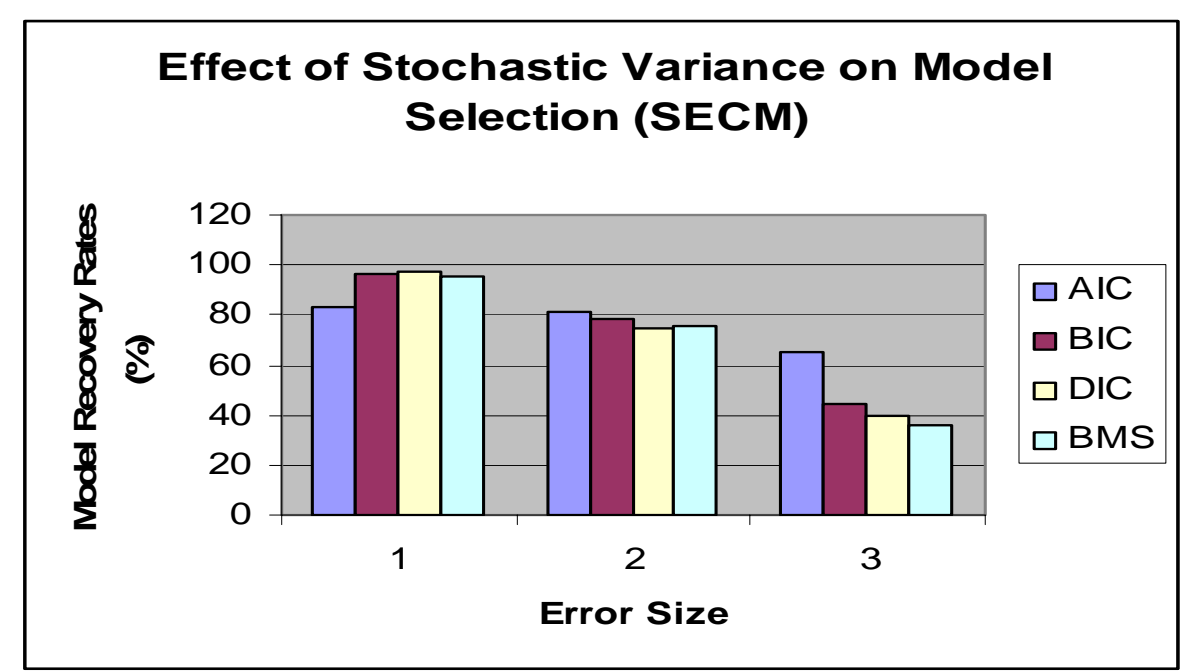

Figure 4: Effects of Stochastic Variance on Model Selection

Second, AIC performed relatively better than the Bayesian Criteria at increased noise levels. Specifically, AIC performs creditably when the error is increased and recovers the true model (SECM) with a recovery rate of 61.4 percent as graphically indicated above. However, as error size decreased from 3 to 1 as graphically illustrated above, the Bayesian criteria (i.e. BIC, DIC and BMS) outperforms the AIC in recovering the true model with recovery rates of 96.5 percent, 96.9 percent and 95 percent respectively. Similarly, Chen et al. (2007) notes the tendency of BIC to perform worse than AIC at high noise levels in a factorial analysis. In a 
comparison of model selection methods, Yang (2003) demonstrates that AIC outperforms $\mathrm{BIC}$ in recovering the true model as noise levels increased in a linear regression analysis framework.

Simulating the effects of sample size and stochastic variance concurrently affirms that a small error and large sample improves recovery of the true asymmetric data generating process and vice versa as graphically illustrated in Figure 5. With a small sample of 50 and an error size of 2.0 the true data generating process was recovered at least 19 percent of the time by all the model selection criteria as illustrated in Figure 5.

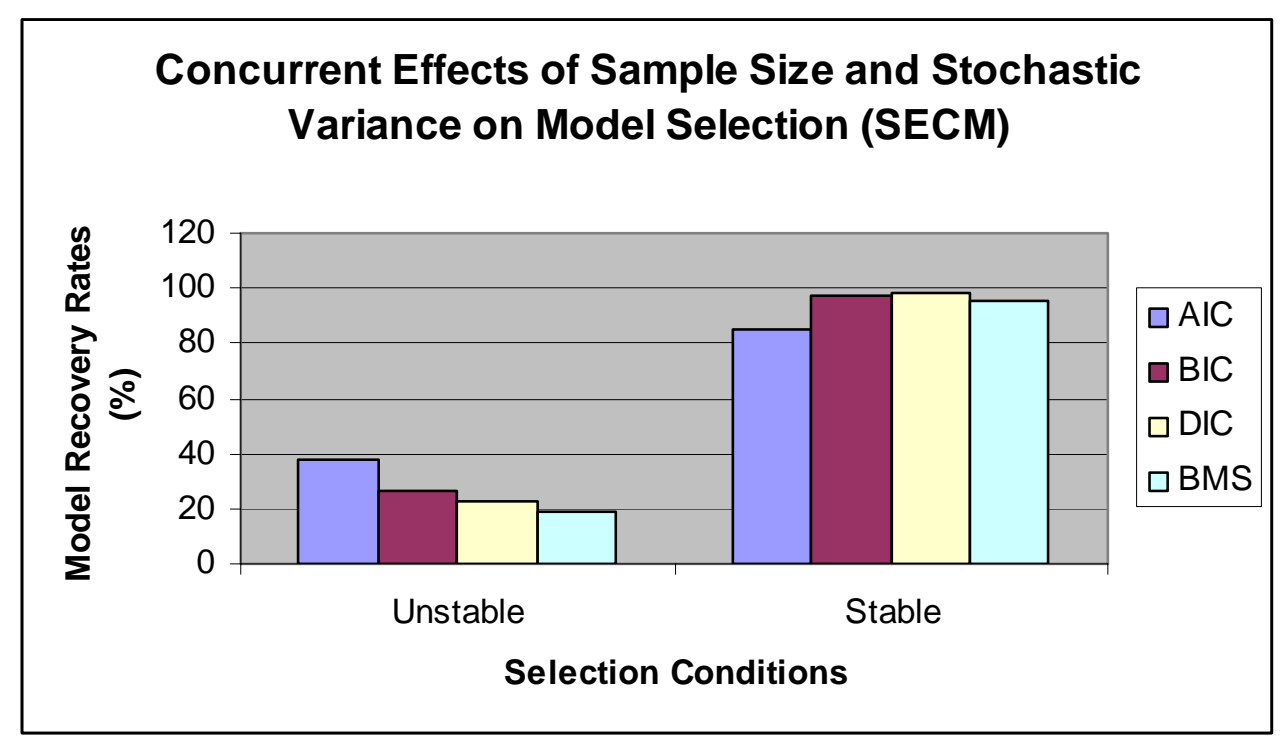

Figure 5: Effects of Stochastic Variance and Sample Size on Model Selection

On the other hand, with a relatively large sample of 150 and error size of 0.5 , at least 84.7 percent the true data generating process was recovered across all the model selection methods as indicated in the Figure 5. Additional information is provided in appendix II. The model recovery rates of the model selection methods are derived under combined conditions of a small sample size of 50 and large error size of 2 (i.e. Unstable conditions), and a relatively large sample size of 150 and a small error size of 0.5 (i.e. Stable conditions ).

Additionally, Figure 6 below illustrates how model fit declined as the error in the model increased. Model fit is measured on the basis of Information Criteria (i.e. AIC, BIC, DIC). The analytical marginal likelihood also declined with increasing noise levels for the true asymmetric data generating process (SECM) as displayed in appendix III. 


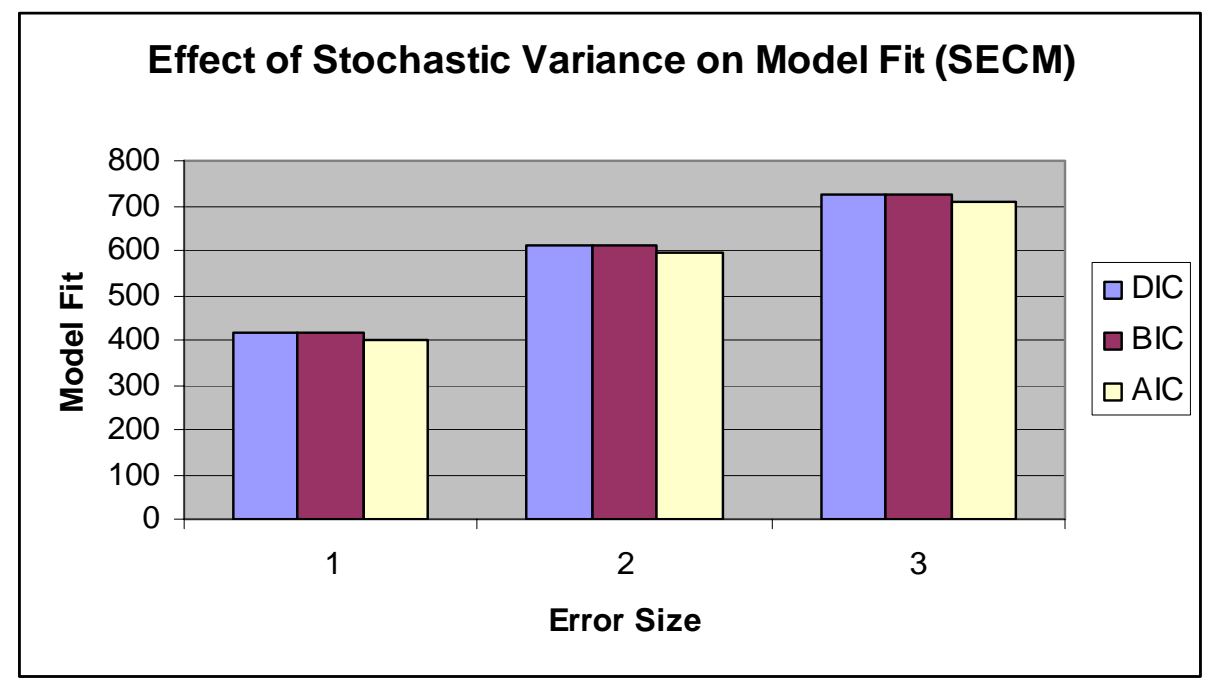

Figure 6: Example of Model fit as a function of Error Size

The model selection methods performed reasonably well in identifying the true model, though their ability to recover the true asymmetric data generating process increases with decreases in error variance. Generally, model fit declined with an increase in stochastic variance. This observation is consistent with theoretical literature. These results underpin the fact that the amount of noise in the model is important in the selection of the true asymmetric data generating process during price transmission analysis.

In summary, this research notes that model selection methods may have difficulty in identifying the true asymmetric model at higher noise levels or alternatively the performances of all model selection methods in recovering the true model may deteriorate with increase in noise levels within the asymmetric price transmission modeling framework.

4.6.3 Monte Carlo Simulation of the Effects of Difference in Asymmetric Adjustment Parameters on Model Selection

This study simulated data of sample size 150 with an error size of 1 from the standard asymmetric price transmission model and asymmetry values $\left(\beta_{2}{ }^{+}, \beta_{2}{ }^{-}\right) \in(-0.25,-0.50)$ or $(-0.25,-0.75)$ are considered for the coefficients of the asymmetric error correction terms. Subsequently, we examine the effect of the increase in difference of asymmetric adjustment parameters on model recovery. Figure 7 illustrates how different model selection methods 
exhibit different relative performance in recovering the true model at different levels of asymmetry.

An increase in the difference between the asymmetric adjustments parameters from 0.25 to 0.5 led to improvement in the model recovery rates of the model selection methods as graphically illustrated. Further details are provided in appendix II.

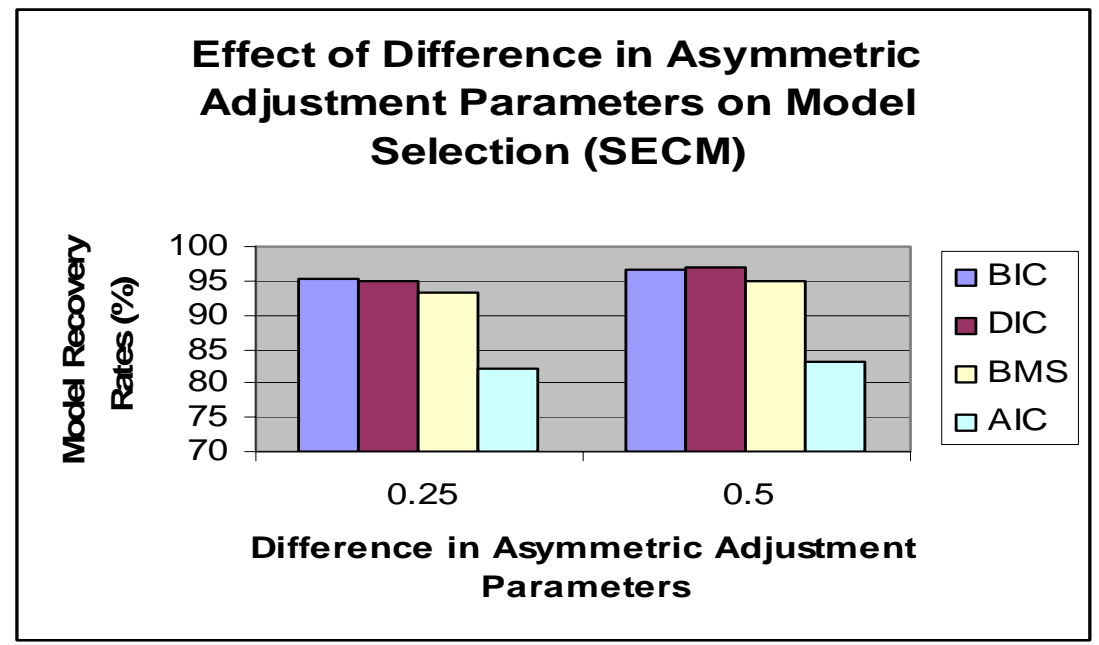

Figure 7: Example of the Effect of the level of Asymmetry

Generally, recovery rates of the Bayesian criteria responds more strongly to increases in the difference between the asymmetric adjustments parameters for the true model. Cook et al. (1999, pp. 155-159) without regards to the concept of the marginal likelihood and information criteria note that the increases in the difference between the asymmetric adjustments speeds (i.e. from 0.25 to 0.50 between the positive and negative components of the error correction terms) have positive effects on the test for asymmetry.

Importantly, another factor which may influence model selection or the recovery of the true data generating process is the difference in asymmetric adjustment parameters as illustrated.

Within the asymmetric price transmission modeling framework, this study has not only shed light empirically on the relative performance of the model selection algorithms of which no studies has been undertaken, but has also established that the Bayesian methods correctly identifies the true asymmetric data generating process. 


\subsection{Overview of the Performance Analysis of the Different Asymmetry Test using Simulated Data generated from the Complex Asymmetric ECM}

A comparative analysis of the models in Table 10 below, illustrates differences in the analytical marginal likelihoods of the selected alternative models which forms the basis for their different rankings provided. The model which fits the simulated data best according to the analytical marginal likelihood is the asymmetric ECM with complex dynamics (CECM), followed by the standard ECM and the variants of the Houck's models. The asymmetric ECM with complex dynamics providing a better fit to the simulated data is not surprising since the true data generating process is the complex asymmetric ECM. The data was simulated from the complex ECM as follows:

$$
\Delta P_{A, t}=3+0.95 \Delta P_{B, t}^{+}+0.20 \Delta P_{B, t}^{-}-0.25 E C T^{+}{ }_{t-1}-0.75 E C T^{-}{ }_{t-1}+\varepsilon
$$

The poor performance of the variants of the Houck's model may be due to the fact that it does not incorporate the error correction mechanism which was incorporated in the true data generating process.

Table10: Complex ECM DGP: Analytical Marginal Likelihood (BMS), BIC, DIC and AIC of selected models (CECM)

\begin{tabular}{|l|l|l|l|l|l|l|l|l|}
\hline & \multicolumn{1}{|l|}{ BMS (Koop,2003) } & \multicolumn{2}{|c|}{ BIC } & \multicolumn{2}{c|}{ DIC } & \multicolumn{2}{c|}{ AIC } \\
\hline Model & Value & Ranking & Value & Ranking & Value & Ranking & Value & Ranking \\
\hline HKD(AR1) & -384.68 & 4 & 764 & 4 & 767 & 4 & 744 & 4 \\
\hline HKD & -380.57 & 3 & 756 & 3 & 757 & 3 & 742 & 3 \\
\hline $\begin{array}{l}\text { CECM } \\
\text { (DGP) }\end{array}$ & -356.09 & 1 & 707 & 1 & 709 & 1 & 686 & 1 \\
\hline SECM & -360.50 & 2 & 714 & 2 & 717 & 2 & 697 & 2 \\
\hline
\end{tabular}

Based on 1000 Replications

Intuitively, the results also suggest that in the presence of cointegration of the series, the Houck's model may not provide a better fit since it excludes information on the long-run equilibrium relationship between the variables. The rankings provided by the marginal likelihood clearly show that given the cointegrated simulated data, none of the models incorporating the error correction mechanism provided the poorest fit to the data. 
Comparison of the static Houck's model in first differences and the error correction model with complex dynamics (CECM) indicate that the improvement in fit could be attributed to the incorporation of the error correction terms which is the main difference between the models. The static Houck's model in sum difference provides the poorer fit to the data with a marginal likelihood of -906. Examination of the residuals of the estimated alternative models indicates a reasonable degree of normality. Additionally, Table 10 above provides model ranking on the basis of approximations of the marginal likelihoods (i.e. BIC and DIC).

On the basis of the BIC values provided above, it is evident that the best model given the complex asymmetric ECM data generating process is the asymmetric ECM model with complex dynamics (CECM), followed by the standard ECM, Houck's model in first differences and the Houck's model in summed differences. The static and dynamic Houck's model in summed differences provided the poorest fit to the simulated data with a BIC of 1760 and 1764 respectively. Notably, the crude approximation of the marginal likelihood (i.e. BIC) provides the same model ranking as the analytical marginal likelihood. This indicates that the BIC can be used as a complement in relation to the marginal likelihood.

Model rankings are also derived from an alternative approximation of the marginal likelihood; Drapers Information Criteria (DIC, Draper, 1995). Model selection involves choosing the model which has the lowest or minimum values of the DIC. The model rankings of the DIC are similar to the Analytical Marginal Likelihood and the Bayesian Information Criteria. On the basis of the DIC values provided above, it is evident that the best fitting model given the artificial data derived from asymmetric ECM with complex dynamics is the complex asymmetric ECM (CECM), followed by the Standard Asymmetric Error Correction Model (SECM) and the variants of the Houck's model in first differences. Again the static and dynamic Houck's model in summed differences provided the poorest fit to the simulated data with a DIC of 1762 and 1767 respectively. The Drapers Information Criteria, Bayesian Information Criteria and the Analytical Marginal Likelihood provide the same model ranking which is consistent with the Akaike Information Criteria as illustrated in Table 10. It is important to appreciate that although both AIC and Bayesian methods (BIC, DIC and BMS) are intended to select good models in a broader sense, there are fundamental differences.

For the models of asymmetric price transmission estimated given the non-stationary cointegrated data, contrasting the fit of the competing models on the basis of the marginal 
likelihood and its approximations (BIC and DIC) indicates that models that incorporate the error correction terms outperforms the static and dynamic variants of the Houck's model. This observation confirms the assertions of Granger and Engle (1987) on theoretical and empirical considerations that in the presence of cointegration, the error correction mechanism exists. Fundamentally, the error correction approach is motivated by the fact that the Houck's models or approaches discussed are not consistent with cointegration between the price series involved (von Cramon-Taubadel, 1998 and von Cramon-Taubadel and Loy, 1999). Essentially, unlike these previous studies the observation that the models which incorporates the error correction term better fits or explains a given set of cointegrated data better than the Houck's models is based on a comparison of marginal likelihood and information theoreticselection criteria.

The sensitivity of the Analytical Marginal Likelihood is examined. For brevity, the prior sensitivity analysis of the asymmetric ECM with complex dynamics (CECM) which outperform the other competing models is illustrated in Table 11 below. The Bayesian result with the original prior used for this model is compared to the classical results as well as the results derived using weak and strong prior variants. The minimal differences in the results of the different estimations are illustrated below in Table 11 below.

Table11: Sensitivity of the Analytical Marginal Likelihood to the Prior (CECM)

\begin{tabular}{|l|l|l|l|l|l|}
\hline \multirow{2}{*}{ Coefficient } & \multirow{2}{*}{$\begin{array}{l}\text { Least } \\
\end{array}$} & \multicolumn{4}{|c|}{ Posterior Means with Prior } \\
\cline { 3 - 6 } & Squares & Weak & Original & Strong & Prior Mean \\
\hline Intercept & 3.011 & 2.990 & 2.994 & 2.985 & 0 \\
\hline$\Delta P_{B, t}^{+}$ & 0.951 & 0.955 & 0.954 & 0.956 & 0 \\
\hline$\Delta P_{B, t}^{-}$ & 0.196 & 0.193 & 0.190 & 0.186 & 0 \\
\hline$E C T^{+}{ }_{t-1}$ & -0.254 & -0.256 & -0.253 & -0.245 & 0 \\
\hline$E C T^{-}{ }_{t-1}$ & -0.746 & -0.744 & -0.746 & -0.745 & 0 \\
\hline & & & & & \\
\hline $\begin{array}{c}\text { Marginal } \\
\text { Likelihood }\end{array}$ & & -357.19 & -356.09 & -355.21 & \\
\hline Basd & & & &
\end{tabular}

Based on 1000 Replications

The comparison illustrated above indicates that the original prior used in our analysis yields results that show minimal difference from that of the least squares estimates, and the 
estimations using weak and strong prior variants respectively. Intuitively, this implies that our estimated models are insensitive to modest modifications of the prior specifications. Else, it would have shown dramatic changes in the posterior estimates.

More importantly, the marginal likelihood is robust to changes in the prior distribution. The marginal likelihood of the weak and strong prior variants for the complex ECM model is 357.19 and -355.21 respectively. These show minimal difference from the marginal likelihood of -356.09 obtained using the original prior. Additionally, it preserves the ranking of the models considered on the basis of analytical marginal likelihood using the variants of strong and weak prior. The prior sensitivity analysis implemented in this study draws from Geweke (2005, pp.157) and Gill (2003, pp.167-168). Noticeably, minimal differences exist between the posterior moments and the least squares estimates for the ECM model with complex dynamics (CECM) in Table 12 below. The estimation result suggests that the data is influential in our analysis.

Table 12: Natural Conjugate Prior, Least squares and Posterior Moments of the CECM (Analytical Method)

\begin{tabular}{|l|l|l|l|l|l|l|}
\hline & \multicolumn{2}{|c|}{ Prior } & \multicolumn{2}{c|}{ squares } & \multicolumn{2}{c|}{ Posterior } \\
\hline Variable & Mean & Std. Error & Mean & Std. Error & Mean & Std. Error \\
\hline Intercept & 0 & 2.129 & 3.011 & 0.242 & 2.994 & 0.137 \\
\hline$\Delta P_{B, t}^{+}$ & 0 & 1.950 & 0.951 & 0.126 & 0.954 & 0.126 \\
\hline$\Delta P_{B, t}^{-}$ & 0 & 1.956 & 0.196 & 0.125 & 0.190 & 0.125 \\
\hline$E C T_{t-1}^{+}$ & 0 & 1.960 & -0.254 & 0.125 & -0.253 & 0.125 \\
\hline$E C T_{t-1}^{-}$ & 0 & 1.953 & -0.746 & 0.126 & -0.746 & 0.126 \\
\hline
\end{tabular}

Based on 1000 Replications

\subsubsection{Empirical Comparison of Model Selection Criteria}

Empirically this study evaluates and compares the performance of the model selection methods in an asymmetric price transmission modeling context when the true data generating process is complex. Unlike previous studies measuring price asymmetry, this study provides empirical evidence on the relative performance of the model selection methods in an 
asymmetric price transmission modeling framework when the true model is complex ${ }^{25}$ as specified in equation 4.6 above.

Our interest in modeling a complex true asymmetric model among other reasons is inspired by recent literature on model selection. Gagne and Dayton (2002) assert that the performance of model selection methods improves when the true model is complex in multiple regression analysis. Markon and Krueger (2004) in a model comparison exercise also noted that the number of variables used to model a phenomenon generally improves the ability to make correct inferences in structural equation modeling.

\subsubsection{Monte Carlo Simulation of the Effects of Sample Size on Model Recovery}

Overall general trends in performance across the different model selection criteria as the sample size increases are similar to those observed when the data was simulated from the standard asymmetric ECM.

Table13: Effects of Sample Size on Model Recovery

\begin{tabular}{|c|c|c|c|c|}
\hline $\begin{array}{l}\text { Sample } \\
\text { Size }\end{array}$ & \multicolumn{4}{|c|}{ Model Fitted } \\
\hline \multirow[t]{6}{*}{50} & & CECM (DGP) & HKD & SECM \\
\hline & Methods & & & \\
\hline & AIC & $118(42.8 \%)$ & $126(6.3 \%)$ & $119(50.9 \%)$ \\
\hline & $\mathrm{BIC}$ & $129(20.7 \%)$ & $133(18.8 \%)$ & $128(60.5 \%)$ \\
\hline & DIC & 131(17.4\%) & $135(22.1 \%)$ & $130(60.5 \%)$ \\
\hline & BMS & $-67(27.6 \%)$ & $-69(21.3 \%)$ & $-67(51.1 \%)$ \\
\hline \multirow[t]{6}{*}{150} & & & & \\
\hline & Methods & & & \\
\hline & AIC & $403(86.7 \%)$ & $435(0 \%)$ & 409 (13.3\%) \\
\hline & $\mathrm{BIC}$ & $421(65.2 \%)$ & $446(0.1 \%)$ & $424(34.7 \%)$ \\
\hline & DIC & $424(68 \%)$ & $448(0.2 \%)$ & $426(31.8 \%)$ \\
\hline & BMS & $-213(75.4 \%)$ & $-226(0.1 \%)$ & $-215(24.5 \%)$ \\
\hline \multirow[t]{6}{*}{500} & & & & \\
\hline & Methods & & & \\
\hline & AIC & $1394(100 \%)$ & $1510(0 \%)$ & $1417(0 \%)$ \\
\hline & $\mathrm{BIC}$ & $1419(100 \%)$ & $1527(0 \%)$ & $1438(0 \%)$ \\
\hline & $\mathrm{DIC}$ & $1422(100 \%)$ & $1529(0 \%)$ & $1440(0 \%)$ \\
\hline & BMS & $-712(100 \%)$ & $-766(0 \%)$ & $-722(0 \%)$ \\
\hline
\end{tabular}

Based on 1000 Replications

\footnotetext{
${ }^{25}$ Complexity is based on the number of asymmetric adjustment parameters.
} 
The ability of the model selection methods to recover the true model (DGP) generally increased with sample size as illustrated in Table 13 above. In general, the relative performance trends of the model selection criteria illustrates that AIC persistently outperforms the Bayesian methods in small samples given the complex asymmetric data generating process. This was also the case when the true model was the standard asymmetric ECM. Using a small sample of 50, the top part of Table 13 indicates that AIC recovers 42.8 percent of the true data generating process. In large samples, we note that both AIC and the Bayesian methods achieve full recovery when the true data generating process is complex. This is in contrast to the recovery rate of 84.8 percent derived from AIC in large samples when the true data generating process was the standard error correction model (SECM).

Obviously, the discussions so far points to the fact that another factor that may influence the performance of the model selection methods is model complexity. Importantly, the Bayesian criteria achieve full recovery in a large sample size of 500 when the true model is complex. Figure 8 below shows the percentage of the simulated data in which the correct model (i.e. CECM) was selected or recovered by the model selection criteria across different sample sizes. Overall this study notes that when the true model is complex, AIC outperforms the Bayesian methods in relatively large sample size of 150 but have similar performance in large sample size of 500. Similarly, previous studies (Lin and Dayton, 1997) found that AIC was superior to BIC when the true model was complex in mixture models. Gagne and Dayton (2002) also observed that AIC are more successful when the true model was relatively complex in multiple regression analysis.

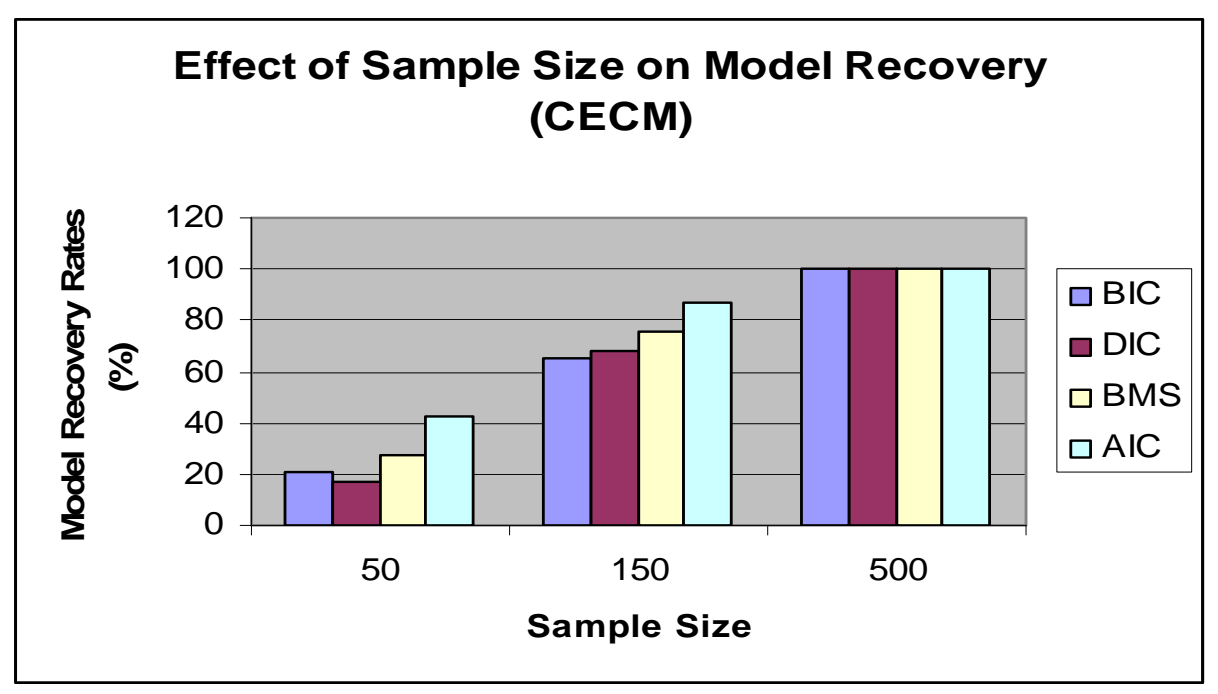

Figure 8: Effects of Sample Size on Model Recovery 
An important point is that comparatively, the model selection methods performed better under stable conditions when the true asymmetric data generating is relatively complex (CECM) than when the standard asymmetric data generating process (SECM) was used under the same stable conditions. This is noted when the recovery rates of Figure 8 are compared with Figure 3 under sample sizes of 150 and 500 respectively. For instance, under a sample size of 500 the model selection methods achieve at least full recovery of the true model when the data generating process is complex but achieves at least 84.8 percent recovery when the model is the standard asymmetric error correction model.

Generally, larger sample sizes improve the ability to make correct inferences about the true asymmetric price transmission model. This research notes that an additional factor that may influence the performance of the model selection criteria in addition to sample size is model complexity (i.e. number of asymmetric adjustment parameters) or the number of informative variables in the model. Intuitively, the increase in model recovery of the true model can also be interpreted as due to an increase in asymmetric information provided by the additional variables.

\subsubsection{Monte Carlo Simulation of the Effects of Noise levels on Model Recovery}

This section details discussion on the effects of error variance on the recovery of the true model and model fit. The data fitting abilities of alternative models are compared in relation to the true model as the error in the data was decreased systematically from 3.0 to 1.0. The results of 1000 Monte Carlo simulations comparing the performance of the model selection methods as error size $(\sigma)$ increased are displayed in Table 14 below.

As expected, recovery rates of the true asymmetric data generating process (i.e. CECM) declined for all model selection methods as the error increased. With a moderate sample size of 150 and an error size $(\sigma)$ of 3 the Houck's model in first difference (HKD) and the standard error correction model (SECM) outperformed the true model (CECM) on the basis of the Bayesian criteria (i.e. BIC, DIC and BMS) as indicated in the top part of Table 14.

As previously mentioned, the improvement in fit and the high model recovery of the Houck's model in first difference over the CECM or the true data generating process may be partly due to the models flexibility to capture random error and not because it accurately approximates 
the model that generated the data. Importantly, the true model incorporates the error correction terms which is ignored in the Houck's specification.

Table14: Effect of Stochastic Variance on Model Recovery

\begin{tabular}{|c|c|c|c|c|}
\hline Error & \multicolumn{4}{|c|}{ Model Fitted } \\
\hline \multirow[t]{6}{*}{3} & & CECM (DGP) & HKD & SECM \\
\hline & Methods & & & \\
\hline & $\mathrm{AIC}$ & $710(19.8 \%)$ & 712 (29.1\%) & 710 (51.1\%) \\
\hline & $\mathrm{BIC}$ & 727 (1.9\%) & $724(58.7 \%)$ & $724(39.4 \%)$ \\
\hline & DIC & $730(1.5 \%)$ & 725 (61. 8\%) & $727(36.7 \%)$ \\
\hline & BMS & $-370(0.7 \%)$ & $-367(67.7 \%)$ & $-368(31.6 \%)$ \\
\hline \multirow{6}{*}{2} & & בזם & PQ & сг्व \\
\hline & Methods & CECIVI (DGP) & HKD & SECM \\
\hline & AIC & 597 (41.6\%) & $604(7.7 \%)$ & $597(50.7 \%)$ \\
\hline & $\mathrm{BIC}$ & $614(8.8 \%)$ & $616(31.4 \%)$ & $612(59.8 \%)$ \\
\hline & DIC & $617(10.8 \%)$ & $618(28.6 \%)$ & $614(60.6 \%)$ \\
\hline & BMS & $-311(7.5 \%)$ & $-311(32.1 \%)$ & $-310(60.4 \%)$ \\
\hline \multirow[t]{6}{*}{1} & & CECM (DGP) & HKD & SECM \\
\hline & Methods & & & \\
\hline & AIC & 402 (88.9\%) & $434(0 \%)$ & 408 (11.1\%) \\
\hline & $\mathrm{BIC}$ & $420(65.7 \%)$ & $446(0.3 \%)$ & $423(34 \%)$ \\
\hline & DIC & $423(67.6 \%)$ & $448(0.5 \%)$ & 425 (31.9\%) \\
\hline & BMS & $-212(74.6 \%)$ & $-225(0.4 \%)$ & $-214(25 \%)$ \\
\hline
\end{tabular}

Based on 1000 Replications

Figure 10 below shows the percentage of the simulated data in which the correct model (i.e. CECM) was selected or recovered among competing models by the model selection criteria as the amount of noise in the data generating process increased.

Comparison of the different selection methods in Figure 9 shows a general trend in which recovery rates decreased with increasing error sizes. In effect, the performance of the model selection algorithms in recovering the true model deteriorates with increasing amount of noise in the true asymmetric price transmission data generating process (CECM). Alternatively, the risk of selecting the false asymmetric model increased at higher noise levels.

With regards to the relationship between the noise levels and the recovery of the true asymmetric data generating process, our findings echo the results of previous studies in other applications. Yang (2003) finds that the recovery rates of the true data generating process 
decreases with increasing noise levels in linear regression models and concludes by noting that selection can yield the wrong model at higher noise levels. Alternatively, with no considerations on model selection methods (i.e. Marginal Likelihood and Information Criteria), Cook et al. ( 1999) using a mathematical analysis and Monte Carlo experiment demonstrate that the test for asymmetry depends on the sample size and the stochastic variance of the true data generating process in recent publications.

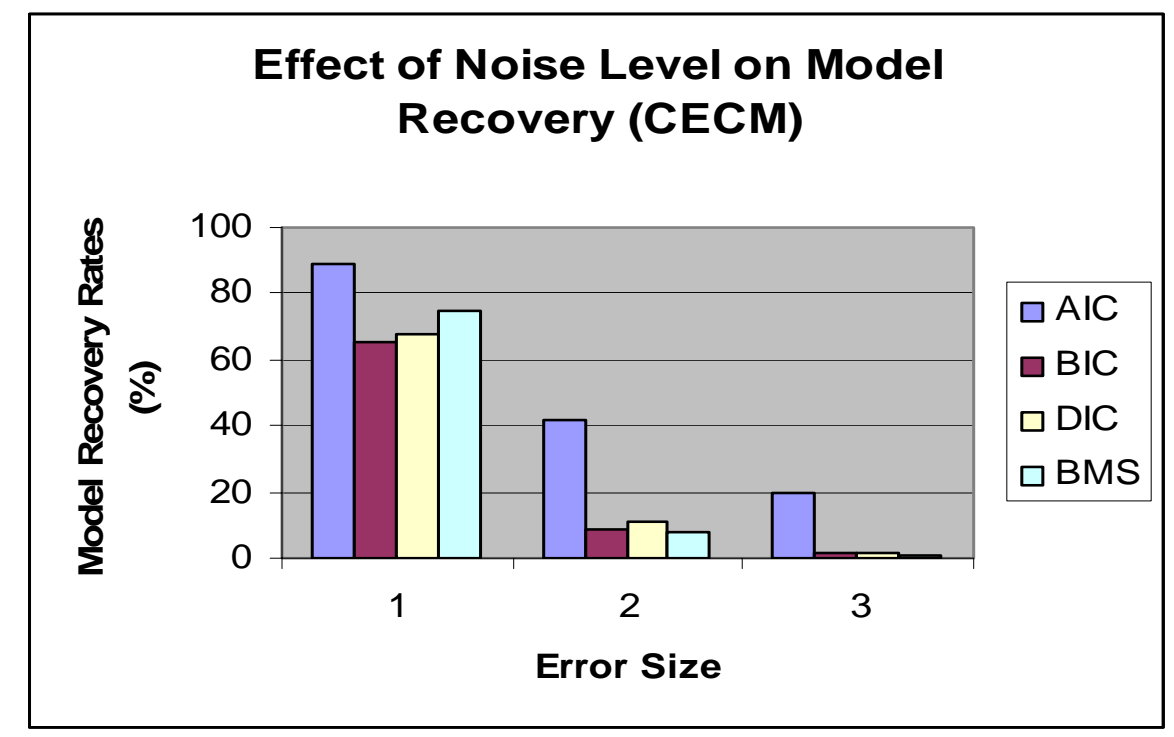

Figure 9: Effects of Noise level on Model Recovery

The Bayesian Criteria (BMS, DIC, and BIC) performed similarly to one another with their recovery rates decreasing substantially as noise levels increased as shown in Figure 9 above. AIC performed relatively better than the Bayesian Criteria at noise levels ranging from 3 to 1 . Specifically, at an error size of 3 , AIC recovers the true model (CECM) with a recovery rate of 19.8 percent as graphically indicated above by outperforming the Bayesian methods. However, as error size decreased from 3 to 1 as graphically illustrated AIC continues to outperform BIC, DIC and BMS. This is in contrast to previous results displayed in the bottom part of Table 9 where the BIC, DIC and BMS outperformed AIC under the same conditions when the true model was not complex (i.e. standard asymmetric error correction model). Similarly, Chen et al. (2007) in factorial data analysis notes the tendency of BIC to perform worse than AIC at high noise levels. In a comparison of model selection methods, Yang (2003) demonstrates that AIC outperforms BIC as noise levels increased in linear regression models. In the context of comparing mixture models, Lin and Dayton (1997) found that AIC 
was superior to BIC when the true model was relatively complex (i.e. complexity is based on the number of parameters).

Importantly, this research notes that the performance of the model selection methods in selecting the true asymmetric price transmission model deteriorates with high levels of noise.

Additionally, we note the tendencies of AIC outperforming BIC when the true asymmetric data generating process is complex (i.e. complexity is based on the number of asymmetric adjustment parameters). Intuitively, complexity influences the performance of the model selection methods in an asymmetric price transmission modeling framework. Simulating the effects of sample size and noise levels concurrently affirms that a small error and large sample improves recovery of the true asymmetric data generating process and vice versa as graphically illustrated in Figure 10.

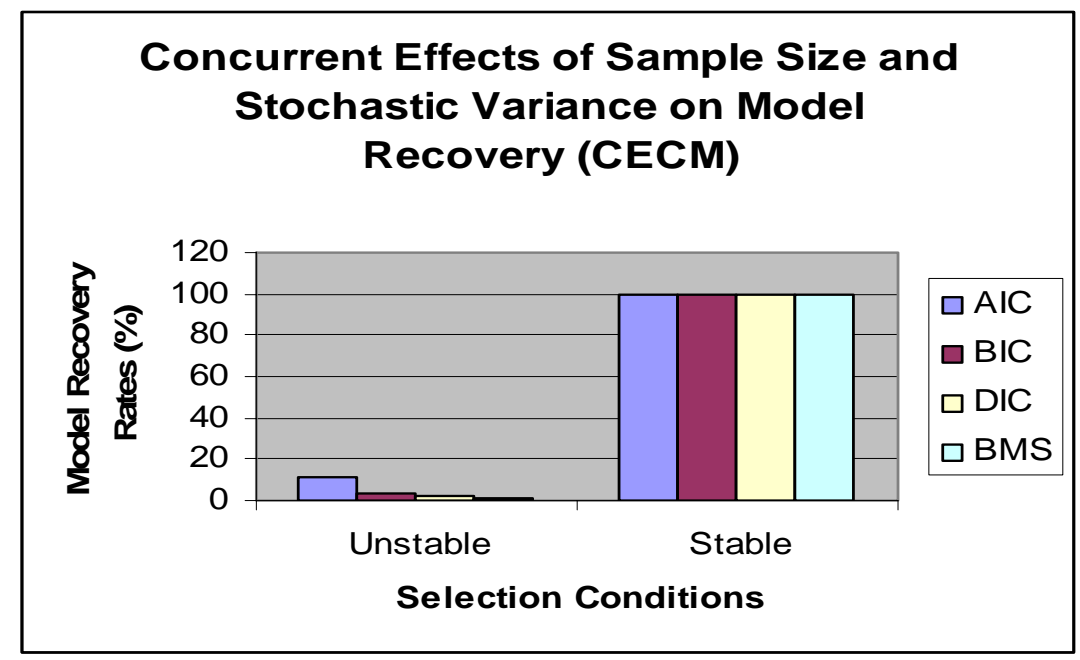

Figure 10: Concurrent Effects of Sample Size and Noise level on Model Recovery

Model recovery rates of the model selection methods are derived under combined conditions of a small sample and large error size (i.e. Unstable conditions) and a relatively large sample and a small error (i.e. Stable condition).

With a small sample of 50 and an error size of 2.0 (Unstable Conditions) the true data generating process was recovered at most 11 percent of the time by all the model selection criteria as illustrated in Figure10 below. On the other hand, with a relatively large sample of 150 and error size of 0.5 (Stable Conditions), at least 99.7 percent of the true data generating 
process was recovered across all the model selection methods as indicated in the Figure 10 (See appendix II for detail Tables).

Obviously the improved performance of the model selection methods can be partly attributed to the fact that the true model is complex. When the true model is complex, all the model selection methods recovered at least 99.7 percent of the true data generating process under stable conditions as shown in Figure 10. However, under the same stable conditions when the true model is not complex (i.e. SECM) all the model selection methods recover at least 84.7 percent of the true data generating process (see Figure 5).

This observation suggests that increase in the number of asymmetric adjustment parameters or variables used to model asymmetry (i.e. complexity of the true model) may have influenced the improved performance or model recovery. Similarly, Markon and Krueger (2004) note that the number of variables used to model a phenomenon generally improves the ability to make correct inferences in structural equation modeling. Gagne and Dayton (2002) assert that the performance of model selection methods improve when the true model is complex in multiple regression analysis.

Additionally, AIC has an edge over the Bayesian methods which only happen when the true asymmetric data generating process is complex. In the previous discussion we noted that the Bayesian methods far outperformed AIC with regards to model recovery when the true model was not complex (i.e. SECM) under similar conditions of a relatively large sample of 150 and error size of 0.5 (Stable Condition).

Similarly, Lin and Dayton (1997) among others demonstrate the superiority of AIC over BIC when the true model was relatively complex (i.e. complexity is based on the number of parameters).

Additionally, the Figure 11 below illustrates how model fit declined as the error in the true model increased. Model fit is measured on the basis of the Information Criteria. The analytical marginal likelihood also declined with increasing noise levels for the true asymmetric data generating process (CECM) as displayed in appendix III. Similarly, Myung (2000) affirms the decline of model fit with increases in error size in a simulation experiment. 


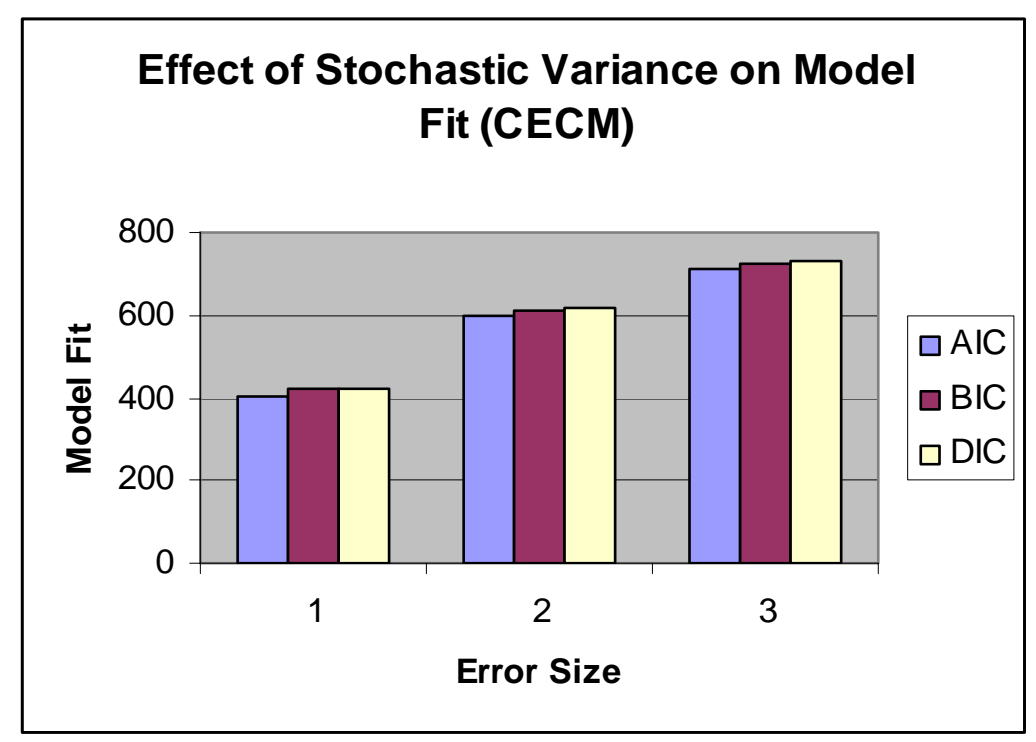

Figure11: Model fit as a function of Noise levels.

4.7.4 Monte Carlo Simulation of the Effects of Difference in Asymmetric Adjustment Parameters on Model Selection (CECM)

This study simulated data of sample size 150 and error size 1 from the error correction model with complex dynamics and asymmetry values of $\left(\beta_{2}^{+}, \beta_{2}^{-}\right) \in(-0.25,-0.50)$ or $(-0.25,-0.75)$ are considered for the coefficients of the asymmetric error correction terms. Subsequently, we examine the effect of the increase in difference of asymmetric adjustment parameters on model recovery.

An increase in the difference in the asymmetric adjustment parameters from 0.25 to 0.5 culminates in an increase in model recovery of the true asymmetric data generating process as illustrated graphically in Figure 12. This study implements a modest modification in the error correction terms of the error correction model with complex dynamics. Recovery rates of the Bayesian criteria responds more strongly to increases in the difference between the asymmetric adjustments parameters.

Similarly, Cook et al. (1999, pp.155-159) without regards to the concept of the marginal likelihood and information criteria note that the increases in the difference in asymmetric adjustments parameters ${ }^{26}$ from 0.25 to 0.50 have positive effects on the test for asymmetry.

\footnotetext{
26 That is the adjustment speeds or positive and negative component of the error correction terms.
} 
Importantly, the performance of the model selection methods in recovering the true data generating process depends on the difference in asymmetric adjustment parameters or speeds.

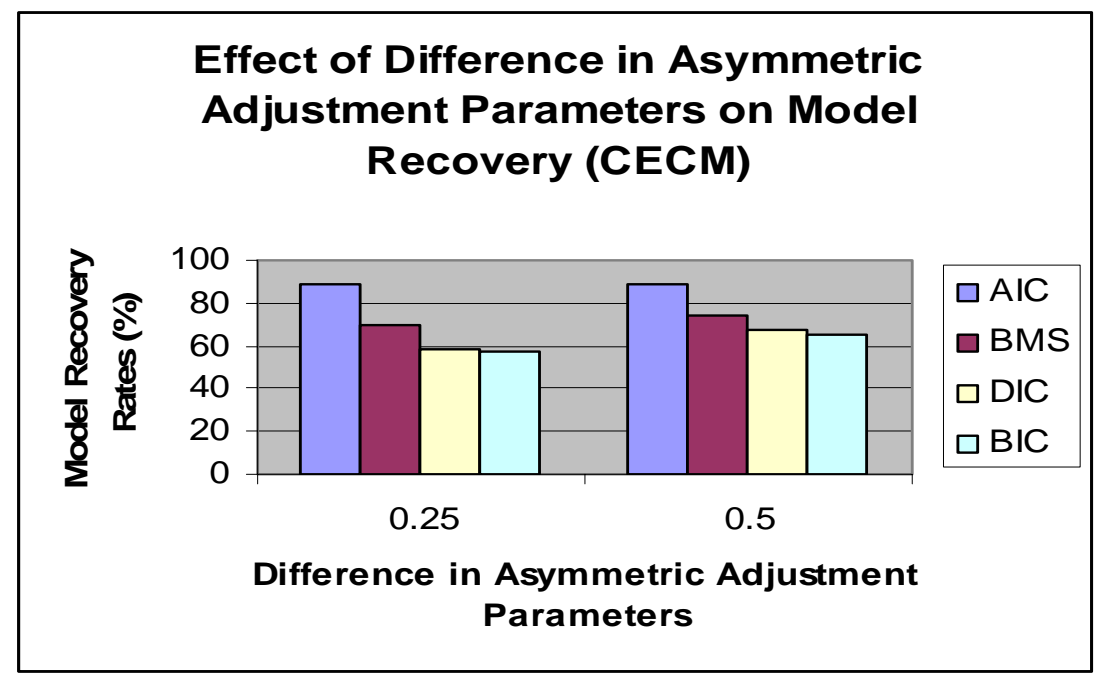

Figure 12: Example of the Effect of the level of Asymmetry

In the asymmetric price transmission framework, the present study has empirically evaluated the relative performance of the model selection algorithms of which little is known and also established that the Bayesian methods do on the average point to the true asymmetric data generating process. Importantly, the procedures implemented in this chapter may apply in a broader sense to a wide range of applications within agricultural sciences when the researcher is confronted with a problem of model selection and comparison.

\subsection{Wolffram- Houck Approaches}

The previous discussions in this chapter assessed the performance of the model selection methods to recover the true asymmetric data generating process when the true data generating process was the standard and complex asymmetric price transmission models. This section widens the range of the asymmetric data generating process used to assess the performance of the model selection methods by including a simple data generating process with asymmetric properties belonging to the Wolffram-Houck approaches. We aim to find out whether the results obtained with regards to the performance of the model selection methods for the complex and standard asymmetric models will hold for a simplistic asymmetric price transmission model. Fundamentally, we examine whether the factors that affect the performance of the model selection methods when the true model was a standard or complex 
asymmetric data generating process also have similar effects when the true model is a simple asymmetric data generating process. Our interest in modeling a simple true asymmetric model among other reasons is inspired by recent literature on model selection. Myung (2000) asserts that in small samples, relatively complex models may over fit a simple true data generating process.

\subsubsection{Model Estimation Results}

The results in this section are derived from Monte Carlo simulations and are based on average across 1000 samples using simulated data with a sample size of 250 . In the proceeding discussion a simple Wolffram model and Houck's model in sum differences are denoted by SWM and HKS respectively. The data was simulated from a simple Wolffram model (see Wolfram 2005) with different levels of asymmetry as follows:

\section{SWM DGP (1):}

$$
P_{A, t}=0.7+0.25 P_{B, t}^{+}+0.75 P_{B, t}^{-}+\varepsilon_{t}
$$

SWM DGP (2):

$$
P_{A, t}=0.7+0.25 P_{B, t}^{+}+0.50 P_{B, t}^{-}+\varepsilon_{t}
$$

As previously mentioned, $P_{B}$ is derived as independent draws from normal distribution with a mean zero and a variance of one and split into increasing and decreasing components (i.e. $P_{B}^{+}$and $\left.P_{B}^{-}\right)$.

Table15: Wolffram DGP (1): Natural Conjugate Prior, Least Squares and Posterior Moments of the SWM (Analytical Method)

\begin{tabular}{|c|l|l|l|l|l|l|}
\hline & \multicolumn{2}{|c|}{ Prior } & \multicolumn{2}{|c|}{ Least squares } & \multicolumn{2}{|c|}{ Posterior } \\
\hline Variable & Mean & Std. Error & Mean & Std. Error & Mean & $\begin{array}{l}\text { Std. } \\
\text { Error }\end{array}$ \\
\hline Intercept & 0 & 1.53 & 0.702 & 0.091 & 0.699 & 0.091 \\
\hline$P_{B, t}^{+}$ & 0 & 1.78 & 0.246 & 0.101 & 0.246 & 0.101 \\
\hline$P_{B, t}^{-}$ & 0 & 1.79 & 0.744 & 0.099 & 0.744 & 0.099 \\
\hline
\end{tabular}

Based on 1000 Replications 
From the estimated results displayed in Table 15 above, the simple Wolffram model (SWM) which best describes the simulated data derived from equation (4.8) on the basis of the marginal likelihood and information criteria tends to provides accurate estimates of the parameters of the artificial data.

From the estimated results it is evident that the difference between the least square estimates and the posterior estimates are minimal. This minimum difference suggests that the data is influential in the results or analysis. In effect, the influence of the prior on the posterior has been demonstrated.

Similarly, minimal differences are observed between the posterior moments and the least squares estimates for the simple Wolffram model when more subtle levels of asymmetry are introduced as exemplified in Table 16 below.

Table16: Wolffram DGP (2): Natural Conjugate Prior, Least Squares and Posterior Moments of the SWM (Analytical Method)

\begin{tabular}{|c|l|l|l|l|l|l|}
\hline & \multicolumn{2}{|c|}{ Prior } & \multicolumn{2}{r|}{ Least squares } & \multicolumn{2}{c|}{ Posterior } \\
\hline Variable & Mean & Std. Error & Mean & Std. Error & Mean & Std. Error \\
\hline Intercept & 0 & 1.456 & 0.699 & 0.091 & 0.698 & 0.091 \\
\hline$P_{B, t}^{+}$ & 0 & 1.708 & 0.250 & 0.101 & 0.251 & 0.101 \\
\hline$P_{B, t}^{-}$ & 0 & 1.699 & 0.503 & 0.099 & 0.499 & 0.099 \\
\hline
\end{tabular}

Based on 1000 Replications

The difference in the asymmetric adjustment parameters was decreased from 0.50 to 025 . That is the positive and negative asymmetric adjustment parameters change from 0.25 and 0.75 in Table 15 above to 0.25 and 0.50 as specified in equation 4.9 and displayed in Table 16 above. Thus, for both strong and subtle levels of asymmetry, the differences between the posterior estimates and the least squares estimations were minimal. The results in Table 16 were derived under a setting where the data was used to construct the prior hyper parameters as detailed in previous discussions. 
Noticeably, the estimation results of the simple Wolffram model (SWM) which provides the best fitting model on the basis of the marginal likelihood and information criteria tends to provide accurate estimates of the parameters of the simulated data as illustrated in equation (4.9). It is important to note that the discussion so far has emphasised the use of moderate sample sizes and we note that using relatively large samples increase the amount of data information and improve the estimation results.

4.8.2 Evaluation of Model Performance using Rankings derived from the Analytical Marginal Likelihood with a Natural Conjugate Prior (SWM)

The proceeding analysis focuses on the most accurate computation of the Marginal Likelihood, the analytical method and draws principally from Geweke (2005, pp.62-65), Koop (2003, pp.15-31), Poirier (1995 pp.542-543) or Zellner (1971, pp.72-75, 1986b). The analytical method explained in detailed in chapter 3 and the formula for computation of the analytical marginal likelihood defined in Zellner (1971) and Koop (2003) and specified in equation (3.25) was used in the construction of the figures in Table 17 below.

For each of the asymmetric price transmission models, Table 17 below reports an estimate of the logarithm of the marginal likelihood. In choosing a single model, this study chooses the one which yields the largest value of the marginal likelihood or provides model rankings using the marginal likelihood as illustrated below. For each of the model selection methods the values provided are the arithmetic mean based on 1000 samples using Monte Carlo simulations.

From the comparison of the models in Table 17 below, this research finds differences in the marginal likelihood of the selected alternative models which forms the basis for their different rankings provided.

The model which fits the simulated data best according to the analytical marginal likelihood is the simple Wolfram model (SWM), followed by the Houck's model in sum differences (HKS) and the error correction models. This observation is consistent with the fact that the true asymmetric data generating process is the simple Wolffram model (WSM). The poor performance of the error correction models model may be partly due to the incorporation of the cointegrating relationship which is originally not included in the true asymmetric 
Wolffram data generating processes. Subsequently, when the Houck's model in sum differences (HKS) and Wolfram's model (SWM) are compared with asymmetric error correction models, a better model fit is achieved. Fundamentally, the rankings provided by the marginal likelihood suggest that the true data generating process belongs to a WolfframHouck type of asymmetry. This partly explains why the Wolffram-Houck models outperform the error correction models. Given the data generated from the Wolffram-Houck procedure, the models incorporating the long run relationship or error correction mechanism produced the poorest fit to the data.

Table 17: Wolffram DGP (1): Analytical Marginal Likelihood (BMS), BIC, DIC and AIC of selected models

\begin{tabular}{|l|l|l|l|l|l|l|l|l|}
\hline & \multicolumn{2}{|c|}{ BMS (Koop, 2003) } & \multicolumn{2}{c|}{ BIC } & \multicolumn{2}{c|}{ DIC } & \multicolumn{2}{c|}{ AIC } \\
\hline Model & Value & Ranking & Value & Ranking & Value & Ranking & Value & Ranking \\
\hline CECM & -359.86 & 4 & 712.67 & 4 & 715.38 & 4 & 691.88 & 4 \\
\hline & & & & & & & & \\
SECM & -358.03 & 3 & 709.30 & 3 & 711.59 & 3 & 689.09 & 3 \\
\hline HKS & -356.25 & 2 & 705.40 & 2 & 707.20 & 2 & 687.07 & 2 \\
\hline $\begin{array}{l}\text { SWM } \\
\text { (DGP) }\end{array}$ & -351.54 & 1 & 699.94 & 1 & 701.75 & 1 & 685.02 & 1 \\
\hline
\end{tabular}

Based on 1000 Replications

Examination of the residuals of the estimated alternative models indicates a reasonable degree of normality. Additionally, Table 17 above provides model ranking on the basis of marginal likelihood approximations such as the Bayesian Information Criteria (BIC). The BIC and the Marginal Likelihood are asymptotically equivalent. The BIC is derived within a Bayesian framework. Model selection entails choosing the model which has the lowest or minimum values of the BIC. The BIC values were computed for the different lags in order to choose the appropriate model. On the basis of the BIC values provided above, it is evident that the best model given the data is the simple Wolffram model (SWM), followed by the Houck's model in sum difference (HKS) and the standard and complex asymmetric error correction models. Notably, for the selected models of asymmetric price transmission, the crude approximation 
of the marginal likelihood (i.e. BIC) provides the same model ranking as the analytical marginal likelihood.

Drapers Information Criteria (DIC, Draper, 1995) which shares the features of the Bayesian Information Criteria as an alternative approximation of the marginal likelihood also forms a basis for ranking the competing models. Model selection involves choosing the model which has the lowest or minimum values of the DIC. The model rankings of the DIC are similar to the Analytical Marginal Likelihood and the Bayesian Information criteria. On the basis of the DIC values provided above, it is evident that the best fitting model given the data is the simple wolfram model (SWM) followed by the Houck's model in sum differences (HKS) and the variants of the error correction models.

The rankings derived by the Bayesian Information Criteria and the Analytical Marginal likelihood are compared to the model rankings of the Akaike Information Criteria. Noticeably, the model rankings provided by the AIC and the BIC or DIC are consistent with marginal likelihood. Model rankings derived from the AIC, BIC, DIC and the Marginal likelihoods are the same. However, it is imperative to note that fundamental differences exist between AIC and BIC, DIC or the marginal likelihood. BIC, DIC and the marginal likelihood are derived within a Bayesian framework and intended to identify the true data generating process or find the most probable model given the data. AIC is not intended to identify the true model exactly but to propose a good approximation of it or to choose the model that is closest to the true model. Alternatively, AIC aims to estimate how well each model with parameters estimated from observed data is expected to predict a new data set of future observations. Although AIC and the Bayesian methods (i.e. BIC, DIC, Marginal Likelihood) are based on different theoretical motivations and objectives, in a broader sense they all have the same aim of identifying good models even if they differ in their exact definition of a good model.

The performance of the model selection criteria are examined using more subtle levels of asymmetry as specified in equation (4.9) and displayed in Table 18 below. The Analytical Marginal likelihood, BIC, DIC and the AIC all correctly identifies the true data generating process when the difference in the asymmetric adjustment parameters decreased from 0.50 to 0.25. Noticeably, both the AIC and the Bayesian methods (i.e. BIC, DIC and the Marginal Likelihood (BMS)) provide the same model ranking. 
The discussion of the computations of the marginal likelihood has implemented analytical computations using informative prior (i.e. Natural Conjugate priors or Normal Gamma) which provides the most accurate value of the marginal likelihood as emphasised by proponents (Zellner, 1971; Koop, 2003; Geweke, 2005). Under this approach, the Baye's factor can be easily specified.

Table 18: Simple Wolffram DGP (2): Analytical Marginal Likelihood (BMS), BIC, DIC and AIC of selected models

\begin{tabular}{|l|l|l|l|l|l|l|l|l|}
\hline & \multicolumn{2}{|c|}{ BMS (Koop, 2003) } & \multicolumn{2}{|c|}{ BIC } & \multicolumn{2}{c|}{ DIC } & \multicolumn{2}{c|}{ AIC } \\
\hline Model & Value & Ranking & Value & Ranking & Value & Ranking & Value & Ranking \\
\hline CECM & -357.83 & 4 & 709.12 & 4 & 711.83 & 4 & 689.24 & 4 \\
\hline SECM & -355.59 & 3 & 704.86 & 3 & 707.12 & 3 & 688.46 & 3 \\
\hline HKS & -353.94 & 2 & 701.02 & 2 & 702.83 & 2 & 687.10 & 2 \\
\hline $\begin{array}{l}\text { SWM } \\
\text { (DGP) }\end{array}$ & -350.27 & 1 & 699.58 & 1 & 701.39 & 1 & 685.66 & 1 \\
\hline
\end{tabular}

Based on 1000 Replications

Importantly, all the model selection algorithms examined clearly identify the correct model out of alternative competing models or on the average point to the true asymmetric data generating process. Our estimation results with all simulated data are accurate for the true model. The results derived from 1000 Monte Carlo simulations in Tables 17 and 18 therefore indicate that information criteria and the marginal likelihood provide a holistic and consistent approach to ranking and selecting among the competing models of asymmetric price transmission. This is better explained by the ability of the alternative Bayesian criteria to select the same true model or provide the same model rankings and the fact that their identified true model is actually the true asymmetric data generating process. Using 1000 Monte Carlo simulations this research has demonstrated that the Bayesian criteria do on average point to the appropriate asymmetry tests in repeated samples. Intuitively, practitioners modeling asymmetry without knowing the true data generating process could emphasize the Bayesian criteria to distinguish between competing models knowing from this study that the Bayesian Criteria do on the average point to the true asymmetric price transmission model. 
An important feature of our results is that they are generally consistent with previous studies focusing on identification of a true data generating process. In support, Berg, Meyer and $\mathrm{Yu}$ (2004) employ a single simulated data generated from a specific stochastic volatility model to establish that the Bayesian methods do point to the true model. Similarly, Myung (2000) demonstrates that the Bayesian methods clearly identify the true data generating process in a cognitive psychology modeling framework.

\subsubsection{Sensitivity of the Analytical Marginal Likelihood to the Prior}

For the sake of brevity, the prior sensitivity analysis of the SWM which outperforms the other competing model is displayed in Table 19 below. The Bayesian result with the original prior used for this model is compared to the classical results as well as the results derived using weak and strong prior variants. The minimal differences in the results of the different estimations are illustrated below in Table 19. The comparison illustrated above indicates that the original prior used in our analysis yields results that is close or shows minimal difference from the least squares estimates and the estimations using a weak and strong prior variants respectively.

Table 19: Wolffram DGP (1): Sensitivity of the Analytical Marginal Likelihood to the Prior (SWM)

\begin{tabular}{|c|c|c|c|c|c|}
\hline \multirow[t]{2}{*}{ Coefficient } & \multirow{2}{*}{$\begin{array}{l}\text { Least } \\
\text { Squares }\end{array}$} & \multicolumn{4}{|c|}{ Posterior Means with Prior } \\
\hline & & Weak & Original & Strong & Prior Mean \\
\hline Intercept & 0.702 & 0.690 & 0.699 & 0.697 & 0 \\
\hline$P_{B, t}^{+}$ & 0.246 & 0.258 & 0.246 & 0.249 & 0 \\
\hline$P_{B, t}^{-}$ & 0.744 & 0.742 & 0.744 & 0.743 & 0 \\
\hline $\begin{array}{l}\text { Marginal } \\
\text { Likelihood }\end{array}$ & & -352.00 & -351.54 & -349.56 & \\
\hline
\end{tabular}

Based on 1000 Replications

Intuitively, this implies that our estimated models are insensitive to modest modifications of the prior specifications. Else, it would have shown dramatic changes in the posterior estimates or summaries. More importantly, the marginal likelihood is robust to changes in the prior distribution. The marginal likelihood of the weak and strong prior variants for the simple 
Wolffram model is -352.00 and -349.56 respectively. These show minimal difference from the marginal likelihood -351.54 obtained using the original prior. Additionally, the ranking of the models considered is preserved when the strong and weak prior variants are used. The prior sensitivity analysis implemented in this study draws from Geweke (2005, pp.157) and Gill (2003, pp.167-168) and Koop (1999) among others.

Similarly, minimal differences in the results of the different estimations are illustrated below in Table 20. The comparison illustrated above indicates that the original prior used in our analysis yields results that is close or shows minimal difference from the least squares estimates and the estimations using a weak and strong prior variants respectively. In effect, the data is still influential in the analysis when more subtle levels of asymmetry are applied as specified in equation (4.9). In addition, the marginal likelihood remains robust to changes in prior distribution and the model rankings are preserved when alternative priors are used.

Table 20: Wolffram DGP (2): Sensitivity of the Analytical Marginal Likelihood to the Prior (SWM)

\begin{tabular}{|l|l|l|l|l|l|}
\hline \multirow{2}{*}{ Coefficient } & \multirow{2}{*}{\begin{tabular}{l} 
Least \\
\cline { 3 - 6 }
\end{tabular}} & \multicolumn{4}{|c|}{ Posterior Means with Prior } \\
\cline { 2 - 6 } & Squares & Weak & Original & Strong & Prior Mean \\
\hline Intercept & 0.699 & 0.695 & 0.698 & 0.692 & 0 \\
\hline$P_{B, t}^{+}$ & 0.250 & 0.252 & 0.251 & 0.261 & 0 \\
\hline$P_{B, t}^{-}$ & 0.503 & 0.497 & 0.499 & 0.493 & 0 \\
\hline & & & & & \\
\hline $\begin{array}{c}\text { Marginal } \\
\text { Likelihood }\end{array}$ & & -350.72 & -350.27 & -349.20 & \\
\hline Based
\end{tabular}

Based on 1000 Replications

\subsubsection{Monte Carlo Simulation of the Effects of Sample Size on Model Selection}

In order to simulate the effects of sample size on model selection, this study considers three sample sizes ranging from small to large corresponding to 50,150 and 500. Using an error size of 1, the data was generated from equation (4.8). The results of the Monte Carlo simulations comparing the performance of the model selection methods are displayed in Table 21 below. 
Table 21: Relative Performance of Model Selection Methods across Sample Size

\begin{tabular}{|c|c|c|c|c|c|}
\hline Sample & \multicolumn{5}{|c|}{ Model Fitted } \\
\hline \multirow[t]{6}{*}{50} & & $\overline{\mathrm{CECM}}$ & SECM & HKS & SWM (DGP) \\
\hline & Methods & & & & \\
\hline & $\mathrm{AIC}$ & $119(8.7 \%)$ & $118(15.6 \%)$ & $118(29.5 \%)$ & $117(46.2 \%)$ \\
\hline & $\mathrm{BIC}$ & $129(2.3 \%)$ & $127(8.5 \%)$ & $125(36.2 \%)$ & $124(53 \%)$ \\
\hline & DIC & $132(1.6 \%)$ & $129(7.5 \%)$ & $126(37.1 \%)$ & $126(53.8 \%)$ \\
\hline & BMS & $-67(1.3 \%)$ & $-66(2.6 \%)$ & $-65(8.3 \%)$ & $-63(87.8 \%)$ \\
\hline \multirow[t]{6}{*}{150} & & CECM & SECM & HKS & SWM (DGP) \\
\hline & Methods & & & & \\
\hline & $\mathrm{AIC}$ & $405(9.5 \%)$ & $404(10.4 \%)$ & $404(13.5 \%)$ & $401(66.6 \%)$ \\
\hline & $\mathrm{BIC}$ & $422(0.5 \%)$ & $419(3.2 \%)$ & $416(15.1 \%)$ & $413(81.2 \%)$ \\
\hline & DIC & $425(0.3 \%)$ & $421(2.6 \%)$ & $418(15.5 \%)$ & $414(81.6 \%)$ \\
\hline & BMS & $-215(0.2 \%)$ & $-214(0.3 \%)$ & $-213(2.8 \%)$ & $-208(96.7 \%)$ \\
\hline \multirow[t]{6}{*}{500} & & CECM & SECM & HKS & SWM (DGP) \\
\hline & Methods & & & & \\
\hline & $\mathrm{AIC}$ & $1403(3.4 \%)$ & $1404(2.2 \%)$ & $1404(2.8 \%)$ & $1393(91.6 \%)$ \\
\hline & $\mathrm{BIC}$ & $1428(0.1 \%)$ & $1425(0.6 \%)$ & $1421(3.4 \%)$ & $1410(95.9 \%)$ \\
\hline & DIC & $1431(0.1 \%)$ & $1428(0.2 \%)$ & $1423(3.4 \%)$ & $1412(96.3 \%)$ \\
\hline & BMS & $-716(0 \%)$ & $-715(0.1 \%)$ & $-713(0.2 \%)$ & $-706(99.7 \%)$ \\
\hline
\end{tabular}

Based on 1000 Replications

Generally, inspection of the recovery rates for the different model selection criteria illustrates the extent to which the true model (SWM) is recovered by each selection criteria across the different sample sizes. In the small sample size of 50, at least 46.2 percent of the true model was recovered across the model selection methods in the top part of Table 21 below. At a sample size of 500, at least 91.6 percent of the true model was recovered by the Bayesian criteria. These results suggest that in small and moderate samples the simple true model was at least recovered from of 46.2 to 66.6 percent because the relatively complex models (i.e. CECM, SECM, and HKS) over fit the data. However, the over fitting effects of the comparatively complex models (i.e. CECM, SECM, and HKS) become less dramatic as sample size increases. Similarly, in an application of model selection methods in cognitive psychology, Myung (2000) notes that the tendency of relatively complex models to over fit a simple true data generating process completely disappears in larger samples.

An inference drawn here is that the tendency of relatively complex models to over fit a simple true asymmetric data generating process will be minimised in larger samples. For the sake of 
brevity, the Houck's model in first difference which was included in all simulations but performed poorly on the basis of all model selection methods( BMS, BIC,DIC and AIC) and was never recovered due to poor model fit is excluded in the Table 21 below.

In comparison with the small sample recovery rates, model recovery of the true model improved significantly when the sample size was large. Despite differences in performance among the model selection criteria, trends holding across the different criteria were evident in the simulation results. In effect, the performance of the model selection methods to select the true model (i.e. recovery rates of SWM) generally increased with increases in sample size from 50 to 500 .

The graph below shows the percentage of the simulated data in which the correct model (i.e. SWM) was selected or recovered by the model selection criteria across different sample sizes. Comparison of the different selection methods in Figure 13 shows a general pattern in which recovery rates increased with increasing sample sizes.

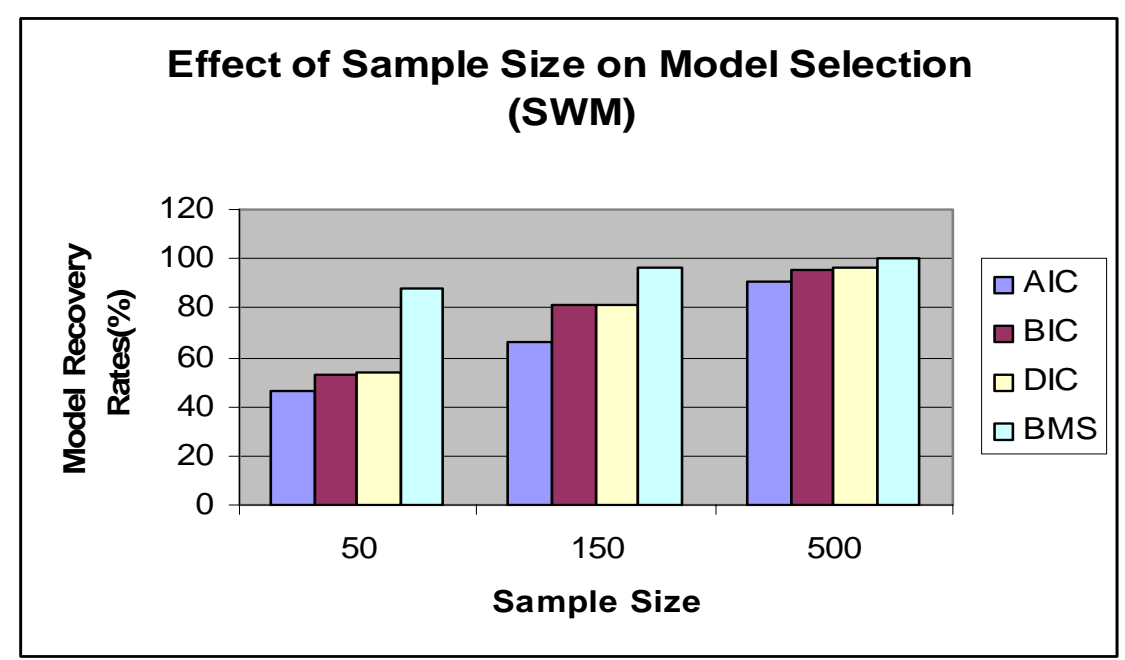

Figure 13: Effects of Sample Size on Model Selection

However, three distinct patterns can also be identified in Figure 13. First, the Bayesian Criteria (BMS, DIC, and BIC) performed similarly to one another with their recovery rates varying strongly as a function of sample size. Second, at large sample size, AIC exhibited a propensity to select complex models. This tendency is observed in the bottom part of Table 21 above where CECM is selected with a recovery rate of 3.4 percent whilst the DIC, BMS, BIC selected the most complex model with a range of (0-0.1 percent). 
These observed patterns are consistent with previous studies on model selection. Ichikawa (1998)'s simulation results in a factor analysis indicated that the ability of AIC to select a true model rapidly increased with sample size but at larger sample sizes it continued to exhibit a slight tendency to select complex models.

Similarly, Markon and Krueger (2004) reviewed existing work on factor analysis and notes that AIC performs relatively well in small samples but is inconsistent and does not improve in performance in large samples whilst BIC in contrast appears to perform relatively poorly in small samples, but is consistent and improves in performance with sample size. Fishler et al. (2002) also investigated the performance of BIC in a factor analysis and their results suggest that BIC performs poorly at small sample sizes, but improves with increasing sample size to eventually choose the correct model with perfect probability.

Third, the Bayesian methods outperformed AIC across the various sample sizes given the simple true model. For both the simple (SWM) and standard (SECM) asymmetric data generating processes the Bayesian methods outperform AIC at larger sample sizes of 50, 150 and 500 as illustrated in Table 8 and Table 21. However, when the true model was complex as demonstrated in Table 13, AIC performed better than or similar to the Bayesian methods across the various sample sizes. It is important to note that it was only when the true model was complex that model selection methods achieved full recovery at a sample size of 500. Lin and Dayton (1997) find that AIC was superior to BIC when the true model was relatively complex (i.e. complexity is based on the number of parameters). Improved performance of the model selection methods when the true model is complex suggest that model complexity influence the performance of the model selection methods.

Although BIC, DIC and BMS performed similarly, there was a slight tendency for DIC to out perform BIC for the variants of samples sizes analysed. Similarly, Markon and Krueger (2004) noted that the DIC outperforms the BIC in a structural equation modeling framework. The tendencies of DIC to out perform BIC in the asymmetric price transmission modeling framework are better explained by the fact that the improved performance of the DIC was the motivation for its development and implementation in Draper (1995). Importantly, BMS outperforms all other selection methods with the highest recovery rates at small, moderate and large sample sizes given the simple true model. 
In summary, the model selection methods performed reasonably well in identifying the true model, though their ability to recover the true asymmetric data generating process increases with increase in sample size as illustrated in Figure 13 above. Intuitively, the results points to the fact that the sample sizes are important in the selection of the true asymmetric data generating process during price transmission analysis. Generally, larger sample sizes might improve the ability of the model selection methods to make correct inferences about asymmetric price transmission models.

The relatively new information criteria; Draper's Information Criteria (DIC, Draper, 1995), which shares the features of the Bayesian Information Criteria, performs similarly to or better than the BIC in asymmetric price transmission modeling framework.

An important feature of our results is that they generally echo existing theoretical and empirical work on the performance of model selection methods in other applications. For instance, investigations with generalized linear models have demonstrated that BIC is consistent, that is, it tends to choose the true model with probability equal to one in large samples but performs poorly in small samples (Hurvich and Tsai, 1990; Bickel and Zhang, 1992). AIC in contrast is not consistent but performs relatively well in small samples.

\subsubsection{Monte Carlo Simulation of the Effects of Stochastic Variance on Model Selection}

In order to simulate the effects of noise level on model selection this study considers three different standard deviations ranging relatively from small to large and corresponding to 1.0, 2.0 and 3.0 across a moderate sample size of 150 generated from equation (4.8). Essentially, the data fitting abilities of alternative models are compared in relation to the true model as the error in the data generating process was increased systematically. The results of 1000 Monte Carlo simulations comparing the performance of the model selection methods as error size $(\sigma)$ increased are displayed in Table 22 below.

Generally, as expected, recovery rates of the true asymmetric data generating process (i.e. SWM) declined for all model selection methods as the error increased. When the true model was simple, the marginal likelihood recovered most of the true model across the various sample size compared to the other selection methods. The improved performance of the marginal likelihood in small samples given the simple data generating process could be partly 
due to the fact that the Bayesian methods rewards parsimony or automatically encodes a preference for simple models.

Table 22: Relative Performance of Model Selection Methods across Noise levels

\begin{tabular}{|c|c|c|c|c|c|}
\hline $\begin{array}{l}\text { Error } \\
\text { Size }\end{array}$ & \multicolumn{5}{|c|}{ Model Fitted } \\
\hline \multirow[t]{6}{*}{3} & & CECM & SECM & $\mathrm{HKS}$ & SWM (DGP) \\
\hline & Methods & & & & \\
\hline & AIC & $709(6.4 \%)$ & $709(21.5 \%)$ & $708(30.7 \%)$ & $707(41.4 \%)$ \\
\hline & $\mathrm{BIC}$ & $727(0.6 \%)$ & $723(6.1 \%)$ & $720(42.3 \%$ & $719(51 \%)$ \\
\hline & DIC & $730(0.4 \%)$ & $725(4.6 \%)$ & $722(43.5 \%)$ & $721(51.5 \%)$ \\
\hline & BMS & $-372(0 \%)$ & $-369(1.6 \%)$ & $-368(21.9 \%)$ & $-365(76.5 \%)$ \\
\hline \multirow[t]{6}{*}{2} & & CECM & SECM & $\mathrm{HKS}$ & SWM (DGP) \\
\hline & Methods & & & & \\
\hline & AIC & $597(8.1 \%)$ & $597(18.3 \%)$ & $596(29.3 \%)$ & $595(44.3 \%)$ \\
\hline & $\mathrm{BIC}$ & $615(0.3 \%)$ & $611(5.3 \%)$ & $608(29.8 \%)$ & $607(64.6 \%)$ \\
\hline & DIC & $618(0.2 \%)$ & $614(4.5 \%)$ & $610(30.3 \%)$ & $609(65 \%)$ \\
\hline & BMS & $-314(0.1 \%)$ & $-311(1.2 \%)$ & $-310(7.9 \%)$ & $-307(90.8 \%)$ \\
\hline \multirow[t]{6}{*}{1} & & CECM & SECM & $\mathrm{HKS}$ & SWM (DGP) \\
\hline & Methods & & & & \\
\hline & AIC & $405(7 \%)$ & $405(11.5 \%)$ & $404(16 \%)$ & $401(65.5 \%)$ \\
\hline & $\mathrm{BIC}$ & $422(0.7 \%)$ & $419(3.2 \%)$ & $416(16.1 \%)$ & $413(80 \%)$ \\
\hline & DIC & $425(0.4 \%)$ & $422(2.9 \%)$ & $418(16.3 \%)$ & $414(80.4 \%)$ \\
\hline & BMS & $-214(0.3 \%)$ & $-212(0.2 \%)$ & $-213(3.2 \%)$ & $-208(96.3 \%)$ \\
\hline
\end{tabular}

Based on 1000 Replications

When the amount of noise in the data generating process was high in the top part of Table 22, the model selection methods recovered at least 41.4 percent of the true data generating process (SWM), allowing the other competing models (i.e. CECM, ECM, and HKS) to over fit the data. At the bottom part of the Table 22, as the noise levels decreased from 3 to 1, the true model was recovered at least 65 percent of the time suggesting that the tendency of the other competing models (i.e. CECM, ECM, HKS) to over fit the data is now reduced or becomes less dramatic.

Generally, we note that the tendency of the relatively complex models (CECM and ECM) to over-fit the data increases with increasing noise levels. Intuitively, higher noise levels make it 
difficult for the model selection methods to identify the true asymmetric model or alternatively the performance of the model selection methods deteriorates with high levels of noise in the asymmetric price transmission modeling framework.

These results are generally consistent with those obtained by experts who studied the effects of noise levels on model selection in other applications such as linear regression models and computer vision applications (See Myung, 2000; Gheissari and Bab-Hadiashar, 2003 ;Yang, 2003). Importantly, Yang (2003) finds that the recovery rates of the true data generating process decreases with increasing noise levels in linear regression models. In conclusion, Yang notes that selection can yield the wrong model at higher noise levels. Alternatively, without regards to model selection methods (i.e. Marginal Likelihood and Information Criteria), Cook et al. (1999) using a mathematical derivation and Monte Carlo experiment demonstrates that the test for asymmetry in particular depends on the sample size and the stochastic variance of the true data generating process in recent publications.

Figure 14 below shows the percentage of the simulated data in which the correct model (i.e. SWM) was selected or recovered among competing models by the model selection criteria as the amount of noise in the data generating process increased. Comparison of the different selection methods in Figure 14 shows a general trend in which recovery rates decreased with increasing error sizes. In effect, the performance of all model selection algorithms analyzed deteriorates with increasing amount of noise in the true asymmetric price transmission data generating process (SWM).

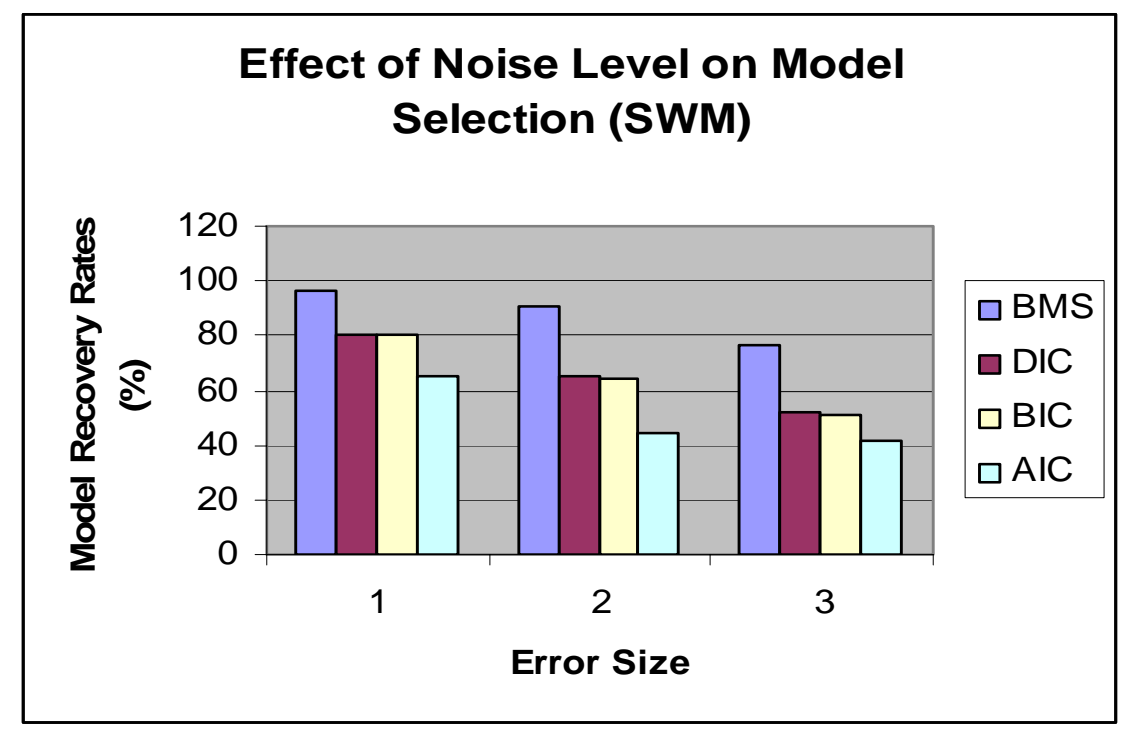

Figure 14: Effects of Stochastic Variance on Model Selection 
Generally, an important trend identified in the graph is that the performance of the model selection methods (BMS, DIC, BIC and AIC) in recovering the simple true model decreased with increasing noise levels in the data generating process. BMS performed relatively better than the BIC, DIC and AIC at increased noise levels. Specifically, BMS performs creditably when the error is increased and recovers the true model (SWM) with a recovery rate of 76.5 percent as graphically indicated above. A decrease in error size from 3 to 1 as graphically illustrated culminates in BMS substantially outperforming BIC, DIC and AIC in recovering the true model with a recovery rate of 96.3 percent respectively.

The model recovery rates of the model selection methods are derived under combined conditions of a small sample and large error size (i.e. unstable conditions) and a relatively large sample and a small error size (i.e. stable conditions). Simulating the effects of sample size and stochastic variance concurrently affirms that a small error and large sample improves recovery of the true asymmetric data generating process and vice versa as graphically illustrated in Figure 15. With a small sample of 50 and an error size of 3.0, the true data generating process was recovered at least 38.8 percent of the time by all the model selection criteria as illustrated in Figure 15.

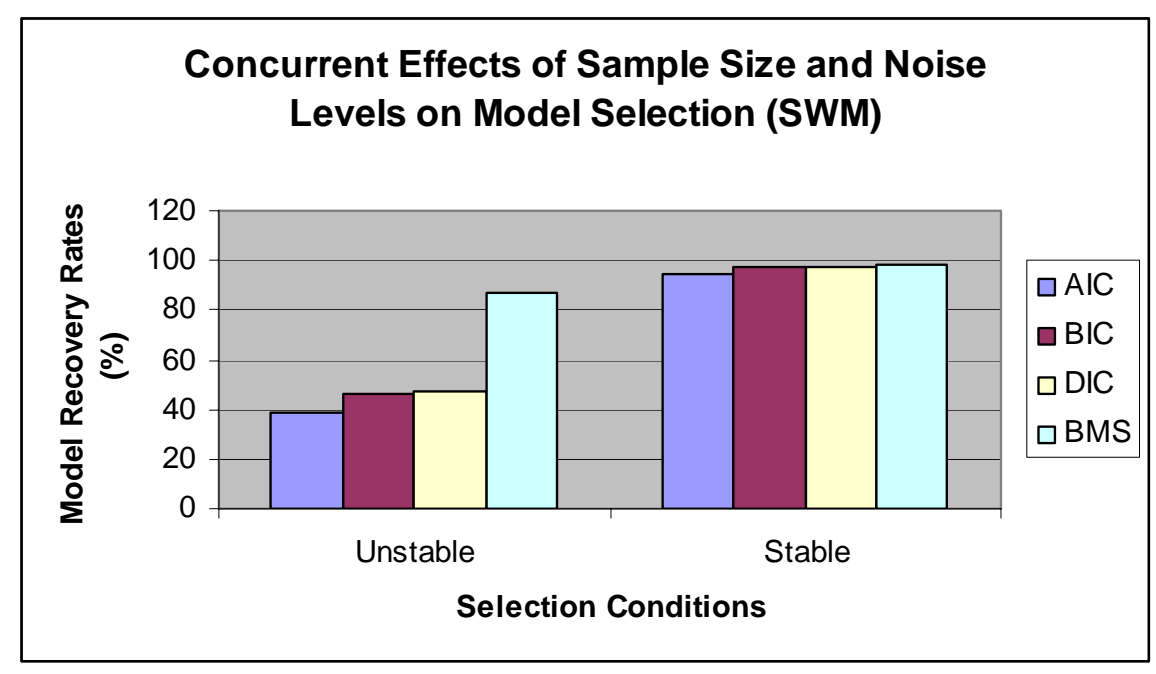

Figure 15: Effects of Stochastic Variance and Sample Size on Model Selection

On the other hand, with a relatively large sample of 150 and error size of 0.5 , the true data generating process (SWM) was recovered at least 94.9 percent across all the model selection methods as indicated in the Figure 15 above. An inference derived here is that stable 
conditions such as a larger sample size and low noise levels will improve the ability of the model selection methods to recover the simple true data generating process.

In previous discussion we noted that the improved performance of the model selection methods can be partly attributed to the fact that the true model is complex. When the true model is complex (CECM), all the model selection methods recover at least 99.7 percent of the true data generating process under stable conditions displayed in Figure 10.

However, under the same stable conditions when the true model is not complex (i.e. SECM) all the model selection methods recover at least 84.7 percent of the true data generating process (see Figure 5). Under stable conditions when the true model is simple, the model selection methods could recover at least 94.9 percent of the true data generating process as illustrated in Figure 15 above.

Additionally, the model selection methods achieved full recovery (i.e. 100 percent) in a large sample size of 500 when the true model is complex (CECM) as illustrated in Figure 8. However, in the case of the standard and simple asymmetric data generating process, full model recovery could not be achieved by the selection methods as in the case when the true data generating process is complex (CECM).

This observation suggests that increase in the number of asymmetric adjustment parameters or variables used to model asymmetry (i.e. complexity of the true model) may have influenced the improved performance or model recovery. Similarly, Markon and Krueger (2004) note that the number of variables used to model a phenomenon generally improves the ability to make correct inferences in structural equation modeling. Gagne and Dayton (2002) assert that the performance of model selection methods improve when the true model is complex in multiple regression analysis.

Figure 16 below illustrates how model fit declines as the error in the true model increases. Model fit is measured on the basis of the Information Criteria (AIC, BIC, DIC). Model fit on the basis of the analytical marginal likelihood (BMS) also declines with increasing noise levels for the true model as indicated in appendix III. 


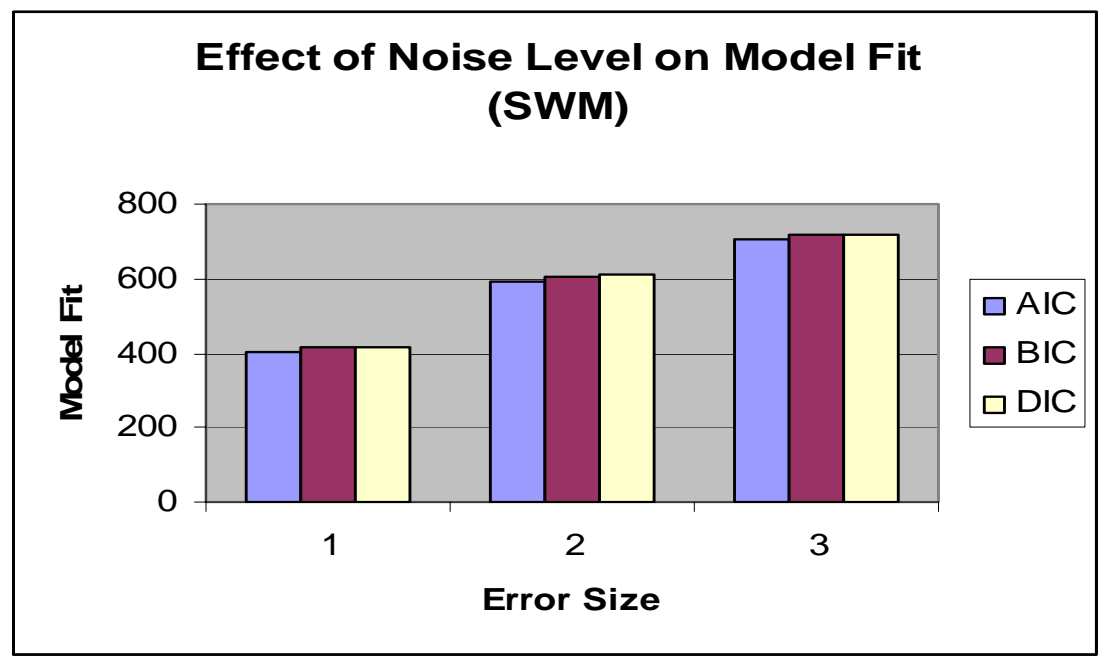

Figure 16: Model Fit as a Function of Noise Levels (SWM)

4.8.6 Monte Carlo Simulation of the Effects of Difference in Asymmetric Adjustment Parameters on Model Selection

This study simulated data of sample size 150 from the standard asymmetric price transmission model and asymmetry values $\left(\beta_{1}^{+}, \beta_{1}^{-}\right) \in(0.25,0.50) \operatorname{or}(0.25,0.75)$ are considered for the coefficients of the asymmetric error correction terms with an error size of 1 . Subsequently, we examine the effect of the increase in difference of asymmetric adjustment parameters on model recovery. Figure 17 below illustrates how different model selection methods exhibit different relative performance in recovering the true model at different levels of asymmetry.

An increase in the difference between the asymmetric adjustments parameters from 0.25 to 0.50 led to improvement in the model recovery rates of the model selection methods as graphically illustrated. Generally, recovery rates of the Bayesian criteria responds more strongly to increases in the difference between the asymmetric adjustments parameters for the true model. In this context, Cook et al. (1999, pp. 155-159) provide a fundamental discussion on the influence of the difference in asymmetric adjustments on the power of the test for asymmetry.

Importantly, another factor which may influence model selection or the recovery of the true data generating process is the difference in asymmetric adjustment parameters as illustrated. 


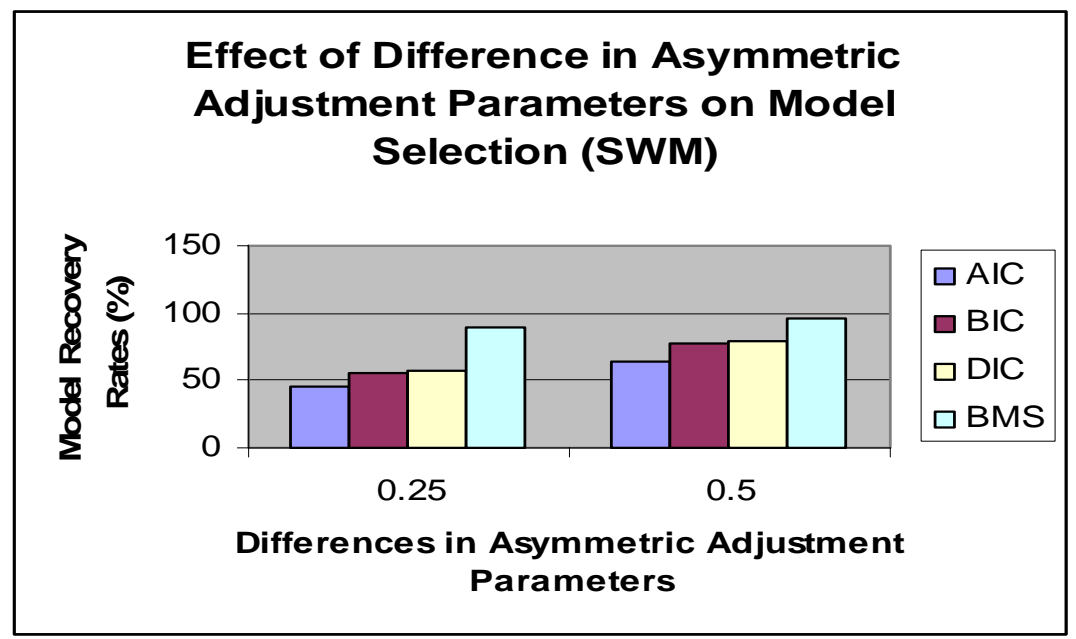

Figure 17: Example of the Effect of the level of Asymmetry

Within the asymmetric price transmission modeling framework, this study has not only shed light empirically on the relative performance of the model selection algorithms of which no studies has been undertaken, but has also established that the Bayesian methods correctly identifies the true asymmetric data generating process within the context of a simple, standard and complex true model.

Previous discussions in this chapter focused on the performance of the model selection methods in identifying the true data generating process, when the true model was derived from a complex and standard asymmetric price transmission approach. The goal of the immediate foregoing discussion was to widen the range of the asymmetric data generating process used to assess the performance of the model selection methods. Subsequently, we considered the performance of the model selection methods when the true data generating was a simple asymmetric data generating process. Importantly, the results indicate that the Bayesian criteria on average do point to the true data generating process when the true model is simple. The results of the model recovery simulations is consistent with previous patterns and trends derived from the model selection methods when the true model is a complex or standard asymmetric data generating process. Importantly, the factors that affect the performance of the model selection methods when the true asymmetric data generating process was standard or complex also holds when the true model was simple. Generally, the over-fitting effects of the other competing models (e.g. complex and standard models) to over fit the simple true data generating process becomes less dramatic as sample sizes increases or 
stochastic variance decreases. Similar observations are noted in Myung (2000) mathematical modeling in cognitive psychology.

\subsection{Summary and Conclusions}

Using a Bayesian analysis, different models of asymmetric price transmission were compared on the basis of the marginal likelihood and information-theoretic selection criteria they attain. The marginal likelihood was ${ }^{27}$ computed using the Analytical Method (BMS) derived in Koop (2003). Model rankings derived from the Analytical Marginal Likelihood (BMS) were compared with those derived from the Bayesian Information Criteria (BIC) and Draper's Information Criteria (DIC).

Bayesian model choice through the concept of the marginal likelihood and information criteria is not only conceptually simple, but also practicable and useful for comparing the models of asymmetric price transmission. This has been demonstrated via Monte Carlo simulations using Analytical computation of the Marginal Likelihood and Information Criteria. Importantly, the empirical comparison of the relative performance of model selection algorithms across the competing asymmetry test is clearly demonstrated when the true data generating process is known.

The findings of this research indicate that model selection algorithms examined clearly identify the correct model out of alternative competing models or on the average point to the true asymmetric data generating process. Our estimation results with all simulated data are accurate for the true model and the analytical marginal likelihood and information criteria provide a holistic and consistent approach to ranking and selecting among the competing models of asymmetric price transmission.

Bayesian Information Criteria which is an approximation of the Marginal Likelihood performs similarly to the Analytical Marginal Likelihood with the same model rankings and can be used as a complementary approach in asymmetric price transmission analysis. The relatively new information criteria; Draper's Information Criteria (DIC, Draper, 1995), which shares the features of the Bayesian Information Criteria, performs similarly to or better than the BIC.

\footnotetext{
${ }^{27}$ All computations in this research were carried out using the R statistical software.
} 
The recovery rates of the true asymmetric model increases with increase in sample size across all model selection methods analysed. Generally, the Bayesian Criteria (BMS, DIC, and BIC) performed similarly to one another and their recovery rates varied more strongly as a function of sample size than the Akaike Information Criteria. The Bayesian Criteria outperforms AIC in large samples when comparing asymmetric price transmission models. Fishler et al. (2002) similarly investigated the performance of BIC in a factor analysis and their results suggest that BIC performs poorly at small sample sizes, but improves with increasing sample size to eventually choose the correct model with perfect probability. Importantly, larger sample sizes might improve the ability of the Bayesian criteria to make correct inferences about asymmetric price transmission models.

The recovery rates of the true asymmetric price transmission model increases with a decrease in stochastic variance across the model selection criteria examined. In effect, the performance of the model selection methods in recovering the true model deteriorates with increases in noise levels within the price transmission modeling framework. Intuitively, this research notes that higher noise levels make it difficult for the model selection methods to identify the true asymmetric model or increase the risk of selecting a false of the true model. Similarly, Gheissari and Bab-Hadiashar (2003) in an analysis of model selection techniques in computer vision applications suggest that the performance of different model selection methods is affected by the amount of noise in the true model as well as model complexity. Alternatively, without regards to model selection methods (i.e. marginal likelihood and information criteria) Cook et al. (1999) using a mathematical derivation and Monte Carlo experiment demonstrates that the test for asymmetry depends on the sample size and the stochastic variance of the true data generating process in recent publications. On the average, model fit declined with an increase in error size for the asymmetric price transmission models analysed.

An important finding in the asymmetric price transmission models is that when the true model is relatively complex, all the model selection methods recovered at least 99.7 percent of the true data generating process under stable conditions. However, under the same stable conditions when the true model is not complex (i.e. SECM and SWM) all the model selection methods recovers at least 84.7 and 94.9 percent of the true data generating process respectively. Moreover, the model selection methods achieved full recovery (i.e. 100 percent) in a large sample size of 500 when the true model is complex (CECM) as illustrated in Figure 8. However in the case of the standard (SECM) and simple (SWM) asymmetric data 
generating process full model recovery could not be achieved by the selection methods as in the case when the true data generating process was complex (CECM). This observation suggests that increase in the number of asymmetric adjustment parameters or variables used to model asymmetry (i.e. the complexity of the true model used in the application) influenced the improved performance or model recovery rates. Similarly, Markon and Krueger (2004) note that the number of variables used to model a phenomenon generally improves the ability to make correct inferences in structural equation modeling framework. The increase in model recovery of the true asymmetric model can also be interpreted as due to an increase in asymmetric information provided by the additional variables. Intuitively, the number of informative variables used to model a phenomenon may improve the recovery of the true data generating process. The increase in the difference between the asymmetric adjustment parameters increases recovery rates of the true asymmetric price model across the model selection criteria investigated. Recovery rates of the Bayesian criteria respond strongly to increases in the difference between the asymmetric adjustments parameters. Cook et al. (1999) without regards to the concept of the marginal likelihood and information criteria note that the increases in the difference in asymmetric adjustments from 0.25 to 0.50 have positive effects on the test for asymmetry. Importantly, another factor which influences recovery of the true data generating process is the difference in asymmetric adjustment parameters.

Prior sensitivity analysis examined the effect of alternative prior distribution on the posterior distribution and the computed Marginal Likelihoods. This involves varying the variance of the prior distribution by a factor of 2 in both directions. The sensitivity analysis indicates that the original prior used in our analysis yields a results that is identical to that of the least squares estimates and the estimations using a weak and strong prior variants. This implies that the estimated models are insensitive to modest modifications of the prior specifications. More importantly, the marginal likelihood is robust to changes in the prior distribution and model rankings are preserved when the variants of prior distribution are used.

In conclusion, the research has shown how model comparison and selection in the competing models of asymmetric price transmission can be systematically achieved using a Bayesian econometric methodology. The usefulness of the marginal likelihood and information criteria has been demonstrated in a price transmission modeling framework with emphasis on their relative performance when the true data generating process is known. The results of our 1000 Monte Carlo simulations can be summarized or outlined as follows: 
Summary of Monte Carlo Simulations

The results indicate that information criteria and the marginal likelihood provides a holistic and consistent approach to ranking and selecting among the competing models of asymmetric price transmission. Our estimation results with all simulated data are accurate for the true model and the marginal likelihood and information criterion clearly identifies the true model. The results of the Monte Carlo simulations further indicate that the performance of the model selection methods in identifying the true asymmetric data generating process depends on the sample size, the amount of noise in the model and the difference in the asymmetric adjustment parameters as well as the complexity of the true asymmetric data generating process. The model recovery rates of the correct asymmetric model provide the basis for measuring the relative performance of the model selection methods. With regards to the relative performance, our results indicate that larger sample sizes improve the ability of the Bayesian Methods (BMS, BIC or DIC) to make correct inferences about asymmetric price transmission models. Unlike the Bayesian Methods, our results suggest that AIC performs well in small samples. The results of our experiments indicate that the performance of all model selection algorithms analysed deteriorate with increasing noise levels for the true asymmetric price transmission data generating process. Importantly, model selection may have difficulty in identifying the true asymmetric model at higher noise levels or alternatively the risk of selecting a false model increases with higher noise levels. In addition, model fit declined with increases in stochastic variance for the price transmission models analysed. Generally, we find similarities between the performance of the marginal likelihood and its approximations (BIC) and (DIC). The performance of the Bayesian criteria improves strongly as a function of sample size and decreases substantially as noise levels increase. Increases in the difference in the asymmetric adjustment parameters and the number of asymmetric parameters use to model asymmetry improve recovery of the true asymmetric model. The Monte Carlo simulation results indicate that a relatively new information criterion; Drapers's Information Criteria (DIC; Draper, 1995), which shares the features of the Bayesian Criteria , performs similarly to or better than the BIC in the price transmission modeling framework. 


\section{An Empirical Comparison of the Power of Tests for Non-Linearity in the Econometric Models of Asymmetric Price Transmission}

The previous chapter has provided evidence of the importance of various conditions in the selection of the true asymmetric data generating process. In this chapter, we demonstrate that the power of the test for asymmetry depends on various conditions such as sample size, difference in adjustment speeds and the amount of noise in the data generating process used in the application. Fundamentally, this section examines whether the conditions that affect the performance of the model selection methods in recovering the true asymmetric data generating process or model are also important in the power test of asymmetry.

Subsequently, we examine the power of the test for asymmetry in the complex von CramonTaubadel and Loy ECM specification and the standard Granger and Lee ECM approach via Monte Carlo Simulation under various conditions when the true data generating process is the standard Granger and Lee Asymmetry.

\subsection{Alternative Approaches of Testing for Asymmetry}

The Granger and Lee asymmetric Error Correction Model data generating process (DGP) can be characterized as follows:

$$
\Delta P_{A, t}=\beta_{1} \Delta P_{B, t}+\beta_{2}{ }^{+} E C T^{+}{ }_{t-1}+\beta_{2}{ }^{-} E C T^{-}{ }_{t-1}+\varepsilon \quad \varepsilon \square N\left(0, \sigma^{2}\right)
$$

Using various sample sizes $P_{A}$ and $P_{B}$ are generated as I (1) non stationary variables that are cointegrated. There exist an equilibrium relationship between $P_{A}$ and $P_{B}$ which produces I (0) stationary series. This equilibrium equation is estimated by least squares and the lagged deviation from this regression denoted by the Error Correction Term $\left(E C T_{t-1}\right)$. The ECT is decomposed into positive and negative deviations using Wolffram segmentation (Granger and Lee, 1989) and plugged into the asymmetric error correction model specified in equation (5.1) above.

Where $\quad E C T=P_{A t}-\beta_{1} P_{B t} \quad$ and

$$
\begin{aligned}
& E C T^{+}{ }_{t-1}=E C T_{t-1} \text { if } E C T_{t-1}>0 \text { and } 0 \text { otherwise, and } \\
& E C T^{-}{ }_{t-1}=E C T_{t-1} \text { if } E C T_{t-1}<0 \text { and } 0 \text { otherwise. }
\end{aligned}
$$


Asymmetry is introduced by allowing the speed of adjustment to differ for the positive and negative components of the Error Correction Term (ECT) since the long run relationship captured by the ECT was implicitly symmetric (see Cook et al, 2000 pp. 224; Cook et al, 2003 pp. 612 ,Cook et al 1999). Symmetry in equation (5.1) is tested by determining whether the coefficients $\left(\beta_{2}^{+}\right.$and $\left.\beta_{2}{ }^{-}\right)$are identical (i.e. $\left.H_{0}: \beta_{2}{ }^{+}=\beta_{2}{ }^{-}\right)$.

The von Cramon-Taubadel and Loy (1996) asymmetric Error Correction Model data generating process (DGP) can be characterized as follows:

$$
\Delta P_{A, t}=\beta_{1}^{+} \Delta P_{B, t}^{+}+\beta_{1}^{-} \Delta P_{B, t}^{-}+\beta_{2}^{+} E C T^{+}{ }_{t-1}+\beta_{2}{ }^{-} E C T^{-}{ }_{t-1}+\varepsilon \quad \varepsilon \square N\left(0, \sigma^{2}\right)
$$

where $\Delta P_{B, t}^{+}$and $\Delta P_{B, t}^{-}$are the positive and negative changes in $P_{B, t}$ and the remaining variables are defined as in equation (5.1).

Von Cramon-Taubadel and Loy (1996) suggested that the $\Delta P_{B, t}$ in equation (5.1) can also be split into positive and negative components to allow for more complex dynamics and applied equation (5.2) to study spatial asymmetric price transmission on world wheat markets. The remaining model variables were defined as in equation (5.1) and formal test of the asymmetry hypothesis using equation (5.2) is: $H_{0}: \beta_{1}{ }^{+}=\beta_{1}{ }^{-}$and $\beta_{2}{ }^{+}=\beta_{2}{ }^{-}$.

Noticeably, since equation (5.2) involves a linear combination of coefficients, a joint F-test can be used to determine symmetry or asymmetry of the price transmission process.

\subsection{A Monte Carlo Analysis of the Power of tests for Asymmetry}

In order to investigate the power of the test for asymmetry under various conditions, a series of Monte Carlo comparison of the two competing models are carried out. These are based on 10000 replications. The data generation process is specified in equation (5.1) with $\beta_{1}$ set to 0.5 and $\left(\beta_{2}{ }^{+}, \beta_{2}{ }^{-}\right) \in(-0.25,-0.50) \operatorname{or}(-0.25,-0.75)$. However, we attempt to detect asymmetry using alternative approaches: the von Cramon-Taubadel and Loy asymmetric ECM and Granger and Lee Model under various conditions. The two methods are compared in terms of their ability to reject the (false) null of symmetric adjustment against the (true) 
alternative of asymmetric adjustment using an F-test of the restricted versus the unrestricted model. The Monte Carlo standard Errors (MCSEs) for these rejection frequencies are given by:

$$
M C S E=\sqrt{\frac{p(1-p)}{M}},
$$

where $\mathrm{p}$ denotes the level of significance and $\mathrm{M}$ is the number of replication used.

The results in tables 23 and 24 below indicate the low power of the conventional F-test in rejecting the incorrect null hypothesis of symmetry.

Table 23: Rejection frequencies based on 10000 Replications

\begin{tabular}{|c|c|c|c|c|c|}
\hline \multicolumn{2}{|c|}{ GL- ECM DGP } & \multicolumn{4}{|c|}{ Model Estimated } \\
\hline \multirow{3}{*}{$\begin{array}{l}\text { Sample } \\
\text { Size }\end{array}$} & \multirow[b]{3}{*}{$\left(\beta_{2}^{+}, \beta_{2}^{-}\right)$} & \multicolumn{2}{|c|}{ GL-ECM } & \multicolumn{2}{|c|}{ vCTL -ECM } \\
\hline & & \multicolumn{4}{|c|}{ Rejection Frequencies } \\
\hline & & $5 \%$ & $1 \%$ & $5 \%$ & $1 \%$ \\
\hline 50 & $(-0.25,-0.50)$ & 0.1173 & 0.0338 & 0.0932 & 0.0255 \\
\hline 150 & $(-0.25,-0.50)$ & 0.3050 & 0.1275 & 0.2350 & 0.0900 \\
\hline 500 & $(-0.25,-0.50)$ & 0.7920 & 0.5630 & 0.7014 & 0.4514 \\
\hline 50 & $(-0.25,-0.75)$ & 0.3150 & 0.1261 & 0.2409 & 0.0801 \\
\hline 150 & $(-0.25,-0.75)$ & 0.8208 & 0.6142 & 0.7358 & 0.5026 \\
\hline 500 & $(-0.25,-0.75)$ & 0.9994 & 0.9978 & 0.9983 & 0.9940 \\
\hline
\end{tabular}

Using 10000 replications the MCSE is 0.00099 at the $1 \%$ level of significance and 0.00218 at $5 \%$ level. Approximate $95 \%$ and $99 \%$ confidence intervals using $\pm 2 M C S E$ for the rejection frequencies can be given as 0.00436 and 0.00198 . The tables 23 and 24 contain the results of the simulations ${ }^{28}$. Table 24 was constructed using a sample size of 150 . For the sake of brevity, we denote the von Cramon -Taubadel and Loy ECM and the Granger and Lee ECM by vCTL and GL respectively.

The Monte Carlo Experimentation indicates the low power of the conventional F-test in rejecting the null of symmetric adjustment in small samples sizes. In effect the Granger and Lee model and the von Cramon-Taubadel and Loy model have lower power in small samples.

\footnotetext{
${ }^{28}$ All simulations were performed using R statistical software.
} 
There is some improvement in power if we increase the difference in asymmetric adjustment parameters from 0.25 to 0.5 in the true model. Similarly, if we decrease the amount of noise in the data generating process (DGP) systematically, an increase in power is also observed in both the complex ECM and standard ECM approaches.

Table 24: Rejection frequencies based on 10000 Replications

\begin{tabular}{|c|c|c|c|c|c|}
\hline \multicolumn{2}{|c|}{ GL- ECM DGP } & \multicolumn{4}{|c|}{ Model Estimated } \\
\hline \multirow{3}{*}{$\begin{array}{l}\text { Error } \\
\text { Size }(\sigma)\end{array}$} & \multirow[b]{3}{*}{$\left(\beta_{2}^{+}, \beta_{2}^{-}\right)$} & \multicolumn{2}{|c|}{ GL-ECM } & \multicolumn{2}{|c|}{ vCTL -ECM } \\
\hline & & \multicolumn{4}{|c|}{ Rejection Frequencies } \\
\hline & & $5 \%$ & $1 \%$ & $5 \%$ & $1 \%$ \\
\hline 3 & $(-0.25,-0.50)$ & 0.0793 & 0.0165 & 0.0662 & 0.0156 \\
\hline 2 & $(-0.25,-0.50)$ & 0.1101 & 0.0364 & 0.0913 & 0.0230 \\
\hline 1 & $(-0.25,-0.50)$ & 0.3080 & 0.1313 & 0.2340 & 0.0913 \\
\hline 3 & $(-0.25,-0.75)$ & 0.1597 & 0.0527 & 0.1266 & 0.0383 \\
\hline 2 & $(-0.25,-0.75)$ & 0.3064 & 0.1320 & 0.2374 & 0.0904 \\
\hline 1 & $(-0.25,-0.75)$ & 0.8202 & 0.6148 & 0.7295 & 0.5041 \\
\hline
\end{tabular}

Based on Sample Size of 150

The results derived show that the factors found by Cook et al. (1999) to affect the power of the Granger and Lee asymmetric model has similar effect on the Von Cramon-Taubadel and Loy ECM specification. In particular this research establishes that the power of the test for asymmetry in the competing models depends on the sample size, the difference between the asymmetric adjustment parameters and the amount of noise in the data generating process.

\subsection{Summary and Conclusions}

In conclusion, the results of our Monte Carlo Experimentation indicates that rejection frequencies increases with increases in sample size, increases in the difference between the asymmetric adjustment speeds and decreases in the amount of noise in the true data generating process used in the application.

Recent studies have shown that the Granger and Lee asymmetric ECM model have lower power in rejecting the null of symmetry. This research has examined the power of alternative 
approach of detecting asymmetry. It has been demonstrated that with small samples or large noise, the power of both the Granger and Lee asymmetric ECM specification and the von Cramon-Taubadel and Loy asymmetric ECM tends to be low. In particular, we have demonstrated that the alternative approaches detect asymmetry at different rates given the same data generating process. The results suggest that various conditions may improve the power of the test for asymmetry. Importantly, the factors that influence the ability of the model selection methods in recovering the true asymmetric data generating process are also influential in the power test of asymmetry. Methodologically, these results reinforce the importance of design informativeness in modeling asymmetries and suggest the conditions under which the power of the test for asymmetry may improve. 


\section{Conclusion, Implications and Directions for Future Research}

\subsection{Conclusions}

This research has presented a comparison of alternative asymmetric price transmission models on the basis of measures of model fit derived from the concept of the marginal likelihood and information criteria using variants of simulated data. The emphasis was on comparing the competing econometric models of asymmetric price transmission using Bayesian techniques which provides a rigorous comparison using a conceptually simple framework.

Besides empirical analysis with an emphasis on relative performance of the model selection methods in an asymmetric price transmission modeling framework, economic theory relating to asymmetric price transmission was briefly reviewed. Theoretical literature on the Bayesian Econometric Methodology was invoked to develop the Bayesian analysis of the asymmetric price transmission models. Conjugate prior (i.e. Normal Gamma) distributions are derived to implement the Bayesian analysis of the asymmetric price transmission regression models. The covariates of the regression models are used to construct the prior distribution in the spirit of Zellner (1986b).

Using a Bayesian analysis, the different models of asymmetric price transmission were compared on the basis of the marginal likelihood they attain. The marginal likelihood was computed using the analytical method (Koop, 2003). Model rankings derived from the marginal likelihood was compared with those derived from approximations of the marginal likelihood such as the Bayesian Information Criteria and the Draper's Information Criteria. Essentially, using variants of simulated data and different model formulations for measuring asymmetric price transmission, this research aimed to investigate the fundamental issue of model selection through implementation of Bayesian Methods. Given the fact that the asymmetric price transmission modeling community continues to develop a wide variety of model specifications, it remains important to develop and understand selection methods that can be applied jointly across these specifications for identification of a true underlying asymmetric data generating process.

This research has demonstrated that Bayesian model choice through the concept of the marginal likelihood and information criteria is not only conceptually simple and theoretically 
logical, but also computationally practicable and useful for comparing the econometric models of asymmetric price transmission.

The findings of this research indicate that model selection algorithms examined clearly identify the correct model out of alternative competing models or on the average point to the true asymmetric data generating process. Estimation results with all simulated data are accurate for the true model and the analytical marginal likelihood and information criteria provide a holistic and consistent approach to ranking and selecting among the competing models of asymmetric price transmission. An inference drawn from these results is that Bayesian model selection techniques works well for asymmetric price transmission model and can identify the underlying true asymmetric data generating process.

The findings of this research indicate that the analytical computation of the marginal likelihood derived in Koop (2003) give identical model rankings when compared with the Draper's Information Criteria and Bayesian Information Criteria of the selected models of asymmetric price transmission, which is a crude approximation of the marginal likelihood under asymptotic conditions given simulated asymmetric data generating process. An inference drawn here is that both the BIC and DIC could be used as a complementary approach to the methods of the marginal likelihood since they all provide the same model ranking or selects the true asymmetric data generating process.

The findings of this research indicate that a relatively new information-theoretic criterion, Draper's Information Criteria (DIC, Draper, 1995), which shares the features of the Bayesian Information Criteria, performs similarly to or better than the BIC in asymmetric price transmission modeling framework. For instance in larger samples, DIC which obtains a recovery rate of 99.5 percent outperforms BIC given the standard asymmetric ECM data generating process. Similarly, when the true model is simple, DIC outperformed BIC in larger samples with a recovery rate of 96.3 percent. Both achieve the same recovery rate of 100 percent given the complex ECM data generating process. The tendency of DIC to outperform BIC is also reflected under stable conditions where DIC outperforms BIC by recovering 97.8 percent of the true model given the standard error correction model data generating process. The tendencies of DIC to perform similar to or better than the BIC under stable conditions is also noted when the true model is the complex or simple asymmetric data generating process. An inference derived from the above results is that DIC is generally preferable to BIC in the 
price transmission modeling framework where either might be useful or applicable, given its better model recovery rates.

The results of our Monte Carlo simulations indicate that the performance of the model selection methods in identifying the true model depends on the sample size, complexity of the true model, amount of noise in the model and the difference between the asymmetric adjustment parameters. The performance of all the model selection methods implemented deteriorates with increases in noise levels within the price transmission modeling framework for the true model. Intuitively, higher noise levels make it difficult for the model selection methods to identify the true asymmetric model. Model recovery rates of the true asymmetric data generating process improves or increases with increases in sample size, increases in the difference between the asymmetric adjustments parameters and increases in the number of asymmetric adjustment parameters (model complexity).

Generally, performance of the Bayesian criteria in recovering the true model improves strongly as a function of increasing sample size and decreases substantially as noise levels increase. For example, as the sample size increased from of 50 to 500 the recovery rates of the true asymmetric model increased from at least 78 percent across the Bayesian Criteria to 98.4 percent given the standard ECM data generating process. Similarly, as sample size increased from 50 to 500 the recovery rates of the true asymmetric data generating process increased from at least 42.8 percent to full recovery across the Bayesian Criteria given the relatively complex true asymmetric error correction model (CECM). Generally, recovery rates of simple asymmetric model (SWM) increased from 46.2 to 95.9 percent as sample sizes increased from 50 to 500. As error variance decreased from 3 to 1 , recovery rates of the true asymmetric model increased from at least 35 percent to a minimum of 95 percent across the Bayesian Criteria given the standard ECM data generating process. When the true model is complex, all the model selection methods recovered at least 99.7 percent of the true data generating process under stable conditions. However, under the same stable conditions when the true model is not complex (i.e. SECM and SWM) all the model selection methods recovers at least 84.7 and 94.9 percent of the true data generating process respectively. Intuitively, larger sample sizes might improve the ability of Bayesian criteria to make correct inferences about asymmetric price transmission models. 
An inference drawn from the observations discussed is that the sample size, difference in the asymmetric adjustment parameters, model complexity and the amount of noise in the model are important in the selection of the true asymmetric data generating process. Modeling asymmetries under stable conditions (e.g. larger sample sizes and lower noise levels) will facilitate selection of the true underlying asymmetric data generating process.

Bayesian analysis of the asymmetric price transmission regression models are implemented using Conjugate prior (i.e. Normal Gamma) distributions. Subsequently, prior sensitivity analysis examined the effect of alternative prior distribution on the posterior distribution and the computed Analytical Marginal Likelihoods. This involves varying the variance of the prior distribution by equal proportions in both directions. The sensitivity analysis indicates that the original prior used in our analysis yields a results that is similar to that of the least squares estimates and the estimations using a weak and strong prior variants. In all cases, there exist minimal differences between the posterior and least squares estimates. This implies that the estimated models are insensitive to modest modifications of the prior specifications. More importantly, the marginal likelihood is robust to changes in the prior distribution and model rankings are preserved when the variants of prior distribution are used. An inference derived here is that reasonable use of prior information or alternatively the application of a natural conjugate (i.e. Normal Gamma) prior distribution yields meaningful results during model selection in asymmetric price transmission modeling framework.

The Monte Carlo Experimentation also indicates that the power of the test for asymmetry depends on sample size, the amount of noise in the model and the difference between the asymmetric adjustment parameters. With small sample size or large noise the performance of the test are low.

In summary, this research has presented an extensive introduction and application of model selection techniques emphasizing the Bayesian criteria which provides a conceptually simple framework for comparing competing models and identifying the true asymmetric data generating process. Importantly, the relative empirical performance of the model selection methods of which little is known in the asymmetric price transmission modeling framework is emphasized. The performance of the marginal likelihood and information criteria are compared by simulating the effects of sample size, amount of noise in the model, increase in the difference between the asymmetric adjustment parameters and increase in the number of 
asymmetric adjustment parameters. The results of the Monte Carlo simulations indicate that the marginal likelihood and information criteria provides a holistic and consistent approach to ranking and selecting among the competing models of asymmetric price transmission. Estimation results with all simulated data are accurate for the true model and the model selection algorithms clearly identify the correct model out of alternative competing models or on the average point to the true asymmetric data generating process. The Monte Carlo simulation results also show that the amount of noise in the model, the sample size, and the difference in the asymmetric adjustment parameters as well as the complexity of the true model are important in identifying the true asymmetric data generating process. In effect, the ability of the model selection methods to recover the true asymmetry data generating process (i.e. Recovery Rates of True Model) increases with increases in the difference between the asymmetric adjustments parameters, increases in sample size, increases in number of asymmetric adjustment parameters (i.e. model complexity) and decreases in the amount of noise in the model. Model fit of all selection methods declined as error increased in the true asymmetric price transmission model. The results of our experiments indicate that the performance of all model selection algorithms analysed deteriorates with increasing noise levels for the true asymmetric price transmission data generating process. Importantly, larger sample sizes and lower noise levels may improve the ability of the Bayesian criteria to make correct inferences about the asymmetric price transmission models. The Monte Carlo simulation results indicate that a relatively new information criterion; Drapers's Information Criteria, which shares the features of the Bayesian Information Criteria, performs similarly to or better than the BIC in the price transmission modeling framework on the basis of the recovery rates of the true asymmetric data generating process. The marginal likelihood give the same model ranking when compared with the BIC and DIC, suggesting that BIC and DIC could be used as a complementary approach.

This research concludes by maintaining the initial assertions that the Bayesian methods through the concept of the marginal likelihood and information criteria is not only logical and conceptually simple but theoretically attractive and useful tools that can be employed to address the problems of model selection in an asymmetric price transmission modeling framework. Using various experiments implemented, the research has shown how model comparison and selection in the competing models of asymmetric price transmission can be systematically achieved using Bayesian criteria. The usefulness of the marginal likelihood and its approximations (i.e. BIC and DIC) has been demonstrated in the price transmission 
modeling framework. Overwhelming evidence derived from the Monte Carlo simulation results is that the Bayesian criteria (i.e. marginal likelihood and information criteria) do on average points to the true asymmetric data generating process. The Monte Carlo simulation results further indicate that the sample size, the difference in the asymmetric adjustment parameters, complexity of the true model (i.e. number of asymmetric adjustment parameters) and the amount of noise in the model are important in recovering the true asymmetric data generating process. The Monte Carlo simulation results also indicate that a relatively new information criterion; Drapers's Information Criteria, which shares the features of the Bayesian Information Criteria performs similarly to or better than the BIC in recovering the true asymmetric price transmission model. Importantly, the conditions that affect the performance of the model selection methods in recovering the true asymmetric data generating process are also influential in the power test of asymmetry.

Considering that in an asymmetric price transmission analysis, there is always the need to select a single model from a set of competing models that best captures the underlying asymmetric data generating process for derivation of policy conclusions since the different specifications employed to detect asymmetric price transmission may lead to different inferences and conclusions (von Cramon Taubadel and Loy, 1999; Capps et al., 2007). It is hoped that the theoretical and empirical analysis presented in this research which exemplifies rigorous model selection using a conceptually simple Bayesian framework which ensures the identification of the true asymmetric data generating process and provides empirical evidence of the relative performance of model selection algorithms will serve as a useful guide in understanding and addressing the methodological issues of model selection during an asymmetric price transmission analysis.

\subsection{Methodological Implications for Asymmetric Price Transmission Modeling}

The study has provided evidence of the usefulness of the Bayesian methods in the asymmetric price transmission modeling framework. The Bayesian criteria do on average points to the true asymmetric data generating process or alternatively perform reasonably well in identifying the true model. The sample size, difference in the asymmetric adjustment parameters, complexity of the true model and the amount of noise in the model are important in the selection of the true asymmetric data generating process. Drapers Information Criteria 
performs similar to or better than the Bayesian Information Criteria in the selection of the true asymmetric model.

First, little is known about the usefulness of the Bayesian Econometric Methods in identifying the true asymmetric data generating process as well as the relative performance of the marginal likelihood and information-theoretic selection criteria in an asymmetric price transmission modeling framework. The comparison provided contributes to knowledge and understanding of the empirical performance of the marginal likelihood and information criteria in an asymmetric price transmission modeling framework. Researchers modeling asymmetry can derive from this research how to implement the Bayesian econometric methodology and draw upon information provided on the general and individual performance patterns of the model selection methods in an asymmetric price transmission context. When modeling asymmetry with no knowledge of the data generating process, Researchers can apply the Bayesian criteria among the competing models knowing from this research that the Bayesian criteria do on the average points to the true asymmetric data generating process. In a broader sense, the agricultural economist when confronted with problems of model selection can draw from implementation approaches and insights from the relative performance analysis or model recovery simulations provided. The concept of Model Recovery Rates or alternatively Selection Power demonstrated in this research via the Monte Carlo simulations can be applied to a wide range of applications or modeling framework to address issues of model selection and comparison in addition to the asymmetric price transmission modeling framework.

Second, the Monte Carlo simulation results of the present study reinforce the importance of design informativeness in implementing asymmetric price transmission analysis. Researchers may use reasonable sample size knowing from this research that increases in sample size improves the ability of the Bayesian criteria to select the true asymmetric data generating process or make correct inferences about the asymmetric price transmission models. Researchers should take into consideration the factors that might influence the ability of the model selection methods to identify the true underlying asymmetric data generating process (e.g. sample size and noise levels among others). The Monte Carlo simulation results also reinforce the importance of another design characteristic: the number of asymmetric adjustment parameters or the number of informative variables. Researchers may increase the number of informative variables use to model asymmetry knowing that asymmetric 
information provided by additional asymmetric variables may improve the ability of the model selection methods to make correct inferences about the true asymmetric data generating process or increase the model recovery rates of the true data generating process. Similarly, in a structural equation modeling framework, Markon and Krueger (2003) demonstrate that the increase in the number of parameters used to model a phenomenon generally improves the ability to make correct inferences. With an informative experimental design or more information in the data, researchers modeling asymmetries can easily identify the true asymmetric model that governs the data.

The findings of the Monte Carlo simulations indicate that the marginal likelihood, BIC and DIC provide the same model ranking and selects the true asymmetric data generating process. Researchers can therefore use the DIC or BIC as complementary approach to marginal likelihood in the asymmetric price transmission modeling framework. Researchers could prefer the relatively new information criteria, DIC to BIC since it performs similar to or better than the BIC on the basis of its model recovery rates of the true asymmetric data generating process.

Researchers could apply prior information in the context of a normal gamma prior distribution (i.e. Natural Conjugate Prior) knowing that reasonable use of prior information is useful in the asymmetric price transmission modeling framework.

The Monte Carlo experimentation also suggest large sample sizes, low noise levels and increase in the difference in asymmetric adjustment speeds as the conditions which will improve the power of the test for asymmetry. In effect, with small sample size or large noise the power of the tests are low. As in every simulation study, the implications of all results discussed should be interpreted with care since model parameters are assigned.

\subsection{Directions for Future Research}

This research focused largely on the methodological problem of model comparison and selection in the econometric models of asymmetric price transmission. The empirical evaluations of the model selection methods have been emphasised in the asymmetric price transmission modeling framework when the true asymmetric data generating process is known. 
The analysis can be extended in a number of ways. Empirical comparison shedding lights on the relative performance of the model selection methods in the asymmetric price transmission modeling framework can be expanded to explore other model selection techniques. The impact of structural breaks and issues of distributional misspecifications on model selection could also be examined in the price transmission framework. With asymmetry as a working hypothesis, future research should focus on model selection problems with emphasis on variants of price transmission models with non linear dynamics. 


\section{References}

Abdulai, A. (2000). Spatial Price Transmission and Asymmetry in the Ghanaian Maize Market. Journal of Development Economics 63, pp. 327-349.

Abdulai, A. (2002). Using Threshold Cointegration to estimate asymmetric Price Transmission in the Swiss Pork Market. Applied Economics 34, pp. 679-687.

Aguero, J. (2004). Asymmetric Price Adjustments and Behaviour Under Risk: Evidence from Peruvian Agricultural Markets. Selected Paper prepared for presentation at the American Agricultural Economics Association Annual Meeting, Denver, Colorado, and July 1-4, 2004.

Aguiar, D. R. D and Santana, J.A. (2002). Asymmetry in Farm to Retail Price Transmission: Evidence from Brazil. Agribusiness 18(1), pp. 37-48.

Aitkin, M. (1991). Posterior Bayes Factor. With Discussion. Journal of the Royal Statistical Society, Series B 53, pp. 111-142

Akaike, H. (1973). Information Theory and an Extension of the Maximum Likelihood Principle. B.N. Petrov and F. Csaki (eds.) $2^{\text {nd }}$ International Symposium on Information Theory: 267-81. Budapest: Akademiai Kiado.

Akaike, H. (1974). A New Look at the Statistical Model Identification. IEEE Transactions on Automatic Control AC-19, pp. 716-723

Albert, J., and Chib, S. (1997). Bayesian Tests and model Diagnostics in Conditionally Independent Hierarchical models. Journal of the American Statistical Association 92, pp. 916925.

Appel, V. (1992). Asymmetrie in der Preistransmission. Agrarwirtschaft Sonderheft 135, pp. 178-213.

Azzam, A. M. (1999). Asymmetry and Rigidity in Farm- Retail Price Transmission. American Journal of Agricultural Economics 81, pp. 525-533.

Bacon, R. W. (1991). Rockets and Feathers: The Asymmetric Speed of Adjustment of UK Retail Gasoline Prices to Cost Changes. Energy Economics 13, pp.211-218.

Bailey, D. and Brorsen, B.W. (1989). Price Asymmetry in Spatial Fed Cattle Market. Western Journal of Agricultural Economics 14 (2), pp. 246-252.

Balke, N.S and Fomby, T. B. (1997). Threshold Co-integration. International Economic Review 38, pp. 627-645.

Balke, N.S., Brown, S.P.A. and Yücel, M.K. (1998). Crude Oil and Gasoline Prices: An Asymmetric Relationship? Federal Reserve Bank of Dallas, Economic Review, First Quarter, pp. 2-11. 
Ball, L. and Mankim, N.G. (1994). Asymmetric Price Adjustments and Economic Fluctuations. The Economic Journal 104, pp. 247-261

Barro, R. J. (1972). A Theory of Monopolistic Price Adjustment. Review of Economic Studies 39, pp. 17- 26.

Bernard, J. C. and Schertz Willet, L. (1996). Asymmetric Price Relationships in the U.S. Broiler Industry. Journal of Agricultural and Applied Economics 28(2), pp. 279-289.

Bickel, P., and Zhang, P. (1992). Variable selection in nonparametric regression with categorical covariates. J. Am. Stat. Assoc. 87, pp.90-97.

Bikker, J.A. (1998). Inflation Forecasting for Aggregates of the EU-7 and EU-14 with Bayesian VAR models. Journal of Forecasting 17, pp.147-165.

Blinder, A.S. (1982). Inventories and Sticky Prices: More on the Micro foundations of Macroeconomics. The American Economic Review 72 (3), pp.334-348.

Blinder, A.S., Canetti., E. R., Lebow, D.E. and Rudd, J.B.(1998). Asking about Prices: A New Approach to Understanding Price Stickiness, Russel Sage Foundation, New York.

Borenstein, S., Cameron, A.C. and Gilbert, R. (1997). DO Gasoline Prices Respond Asymmetrically to Crude Oil Price Changes? Quarterly Journal of Economics 112, pp. 305339.

Bos, C. (2002). A Comparison of Marginal Likelihood Computation Methods. Proceedings in Computational Statistics 2002 pp. 111-117.

Boyd, M. S. and Brorsen, B. W. (1998). Price Asymmetry in the US Pork Marketing Channel. North Central Journal of Agricultural Economics 10, pp.103- 109.

Box, G. E. P. (1979). Robustness in the Strategy of Scientific Model-Building. pp. 201-236 in Robustness in Statistics, edited by Robert L. Lauer and Graham N. Wilkinson. New York: Academic Press.

Bozdogan, H. (1987). Model Selection and Akaike's Information Criterion (AIC): The General Theory and Its Analytical Extensions. Psychometrika 52(3), pp. 345-370.

Brown, S.P.A. and Yücel, M. K. (2000). Gasoline and Crude Oil Prices: Why the Asymmetry? Federal Reserve Bank of Dallas, Economic and Financial Review, Third Quarter, pp. 23-29.

Buckle, R. A. and Carlson, J. A. (2000). Inflation and Asymmetric Price Adjustment. Review of Economics and Statistics 82(1), pp. 157- 160.

Capps, O. and Sherwell, P. (2007). Alternative Approaches in Detecting Asymmetry in FarmRetail Prices Transmission of Fluid Milk. Journal of Agribusiness 23(3), pp.313-331.

Carlin, B. and Chib, S. (1995). Bayesian Model Choice via Markov Chain Monte Carlo Methods, Journal of the Royal Statistical Society, Series B, 57, pp. 473-484. 
Carlin, B. and Louis, T. (2000). Bayes and Empirical Bayes Methods for Data Analysis. Boca Raton: Chapman and Hall/CRC

Carlton, D. W. (1986). The Rigidity of Prices. The American Economic Review 76 (4), pp. 637- 658

Chavas, J. P. and Mehta, A. (2004). Price Dynamics in a Vertical Sector: the Case of Butter. American Journal of Agricultural Economics, 86 pp. 1078-1093.

Chen, L., Giannakouros, P. and Yang, Y. (2007). Model Combining in Factorial Data Analysis. Journal of Statistical Planning and Inference 137 (9), pp.2920-2934.

Congdon, P. (2003). Applied Bayesian Modelling. West Sussex: John Wiley and Sons Ltd.

Cook, S., Holly, S. and Turner, P. (1999). DHSY Revisited: The Role of Asymmetries. Applied Economics 31, pp. 775-778.

Cook, S., Holly, S. and Turner, P. (1999). The Power of Tests for Non-Linearity: The Case of Granger-Lee Asymmetry. Economics Letters 62, pp.155-159.

Cook, S., Holly, S. and Turner, P. (2000). The Power of Tests for Non-linearity: The Escribano-Pfann Model. Computational Economics 15, pp. 223-226.

Cook, S. (2000). Frequency Domain and Time Series Properties of Asymmetric Error Correction Models. Applied Economic 32, pp. 297-307.

Cook, S. and Holly, S. (2002) Threshold Specification for Asymmetric Error Correction Models. Applied Economics Letters 9, pp. 711-13.

Cook, S. (2003). A Sensitivity Analysis of Threshold Determination for Asymmetric Error Correction Models. Applied Economic Letters 10, pp. 611-616.

Cox, D.R. (1961). Tests of Separate Families of Hypotheses. Proceedings of the Fourth Berkeley Symposium on Mathematical Statistics and Probability. Berkeley: University of California Press. pp. 105-123.

Dalagaard, P. (2002). Introductory Statistics with R. New York, USA: Springer-Verlag.

Damania, R. and Yang, B.Z. (1998). Price Rigidity and Asymmetric Price Adjustment in a Repeated Oligopoly. Journal of Institutional and Theoretical Economics 154, pp. 659-679.

Dayton, M.C (2003). Model Comparisons Using Information Measures. Journal of Modern Applied Statistical Methods 2(2), pp. 281-292.

Draper, D. (1995). Assessment and Propagation of Model Uncertainty. Journal of Royal Statistical Society, Series B (Methodological) 57, pp 45-97.

Dutta, S., Bergen, M., Levy, D. and Venable, R.(1999). Menu Costs, Posted Prices, and Multiproducts Retailers. Journal of Money, Credit and Banking 31(4), pp. 683-703. 
Enders, W. (2004). Applied Econometric Time Series. New Jersey: John Wiley and Sons Ltd.

Enders, W. and Granger, C.W.J. (1998). Unit Root Test and Asymmetric Adjustments with an Example Using the Term Structure of Interest Rates. Journal of Business and Economic Statistics 16, pp. 304-311.

Enders, W. and Siklos, P.L. (2001). Cointegration and Threshold Adjustment. Journal of Business and Economic Statistics 19, pp 166-167.

Engle, R. F. and Granger, C. W. J. (1987). Co-integration and Error Correction: Representation, Estimation and Testing. Econometrica 55, pp. 251-276.

FAO (2003). Market Integration and Price Transmission in Selected Food and Cash Crop Markets of developing Countries: Review and Applications, by Rapsomanikis, G., Hallam, D., and P. Conforti, in Commodity Market Review 2003-2004. Commodities and Trade Division, FAO, Rome.

Faraway, J.J. (2005). Linear Models with R. Boca Raton: Chapman and Hall/CRC

Farrell, M. J. (1952). Irreversible Demand Functions. Econometrica 20, pp. 171-186.

Felix, R.M. and Nunes, L. C. (2003). Forecasting Euro Area Aggregates with Bayesian Var and Vecm Models. Bank of Portugal Working paper.

Fishler, E., Grosmann, M., and Messer, H. (2002). Detection of Signals by Information Theoretic Criteria: General Asymptotic Performance Analysis. IEEE Trans. Signal Process 50, pp.1027-1036.

Fox, John (1997). Applied Regression Analysis, Linear Models, and Related Methods. Thousand Oaks, CA: Sage.

Freebairn, J. W. (1984). Farm and Retail Food Prices. Review of Marketing and Agricultural Economics 52(2), pp.71-90.

Frost, D. and Bowden, R. (1999). An asymmetry Generator for Error Correction Mechanism, with Application to Bank Mortgage- Rate Dynamics. Journal of Business and Economic Statistics 17(2), pp. 253-263.

Gagne, P. and Dayton, C.M. (2002). Best Regression Model Using Information Criteria. Journal of Modern Applied Statistical Methods 1, pp.497-488.

Gardner, B.L. (1975). The Farm-Retail Prices Spread in a Competitive Food Industry, American Journal of Agricultural Economics (1975) pp. 399- 409.

Gauthier, W. M. and Zapata, H. (2001). Testing Symmetry in Price Transmission Models, Louisiana State University, Department of Agricultural Economics and Agribusiness, Working Paper.

Gelfand, A and Dey, D. (1994). Bayesian Model Choice: Asymptotic and Exact Calculations. Journal of Royal Statistical Society, Series B56, pp. 501-514. 
Gelman, A., Carlin, B., Stern, H and Rubin, D. (2004). Bayesian Data Analysis. Boca Raton: Chapman \& Hall/CRC.

Geweke, J. (2005). Contemporary Bayesian Econometrics and Statistics. New Jersey: John Wiley and Sons.

Gheissari, N. and Bab-Hadiasher, A. (2004). Effect of Noise on Model Selection Criteria in Visual Applications. Pattern Recognition 2(23-26), pp. 229-232.

Gill, Jeff (2002). Bayesian Methods: A Social and Behavioral Sciences Approach. Boca Raton: Chapman \& Hall/CRC.

Gollnick,H.(1972). Zur statistischen Schätzung und Prüfung irreversibler Nachfragefunktione n. Agrarwirtschaft 21, pp. $227-231$.

Goodwin, B.K. and Holt, M.T. (1999). Asymmetric Adjustment and Price Transmission in the US Beef Sector. American Journal of Agricultural Economics 81, pp. 630-637.

Goodwin, B. K. and Harper, D. C. (2000). Price Transmission, Threshold Behaviour and Asymmetric Adjustment in the U.S. Pork Sector. Journal of Agricultural and Applied Economics 32, pp. 543-553.

Goodwin, B. K. and Piggott, N.E. (2001). Spatial Market Integration in the Presence of Threshold Effects. American Journal of Agricultural Economics 83(2), pp. 302- 317.

Granger, C.W. J. and Newbold, P. (1974). Spurious Regressions in Econometrics. Journal of Econometrics 2, pp. 111-120.

Granger, C.W. J. and Lee, T.H. (1989). Investigation of Production, Sales and Inventory Relationships using Multicointegration and non-symmetric Error Correction Models. Journal of Applied Econometrics 4, pp. 135- 159.

Granger, C.W. J. and Teräsvirta, T (1993). Modelling Nonlinear Economic Relationships. Oxford University Press.

Griffith, G. R. and Piggot, N. E. (1994). Asymmetry in Beef, Lamb and Pork Farm-Retail Price Transmission in Australia. Agricultural Economics 10, pp. 307-316

Hahn, W.F. (1990). Price Transmission Asymmetry in Pork and Beef Markets. The Journal of Agricultural Economics Research 42(4), pp. 21-30.

Hannan, T.H. and Berger, A.N. (1991). The Rigidity of Prices: Evidence from the Banking Industry. The American Economic Review 81(4), pp. 938-945.

Hansen, B.E. and Seo, B. (2002). Testing for Two-Regime Threshold Cointegration in Vector Error Correction Models. Journal of Econometrics 110, pp. 293-318.

Hansmire, M. R. and Schertz Willet, L. (1992). Price Transmission Processes: A Study of Price Lags and Asymmetric Price Response Behaviour for New York Red Delicious and McIntosh Apples. Cornell University 
Heien, D.M. (1980). Markup Pricing in a Dynamic Model of Food Industry. American Journal of Agricultural Economics 62, pp. 10-18.

Hepple, L.W, (1995a). Bayesian Techniques in Spatial and Network Econometrics: Model Comparison and posterior Odds. Environment and Planning A27, pp 447-469.

Hepple, L.W, (2003). Bayesian Model Choice in Spatial Econometrics. Paper for LSU Spatial Econometrics Conference, Baton Rouge.

Holly, S., Turner P. and Weeks, M. (2003). Asymmetric Adjustment and Bias in Estimation of an Equilibrium Relationship from a Co-integrating Regression. Computational Economics 21, pp. 195-202.

Houck, J.P. (1977). An Approach to Specifying and Estimating Nonreversible Functions. American Journal of Agricultural Economics 59, pp. 570-572.

Hurvich, C. M., and Tsai, C-L. (1990). The Impact of Model Selection on Inference in linear Regression. Am. Stat. 44, pp. 214-217.

Ichikawa, M. (1988). Empirical Assessments of AIC Procedure for Model Selection in Factor Analysis. Behaviormetrika 24, pp. 33-40.

Ihaka, R., and Gentleman, R. (1996). R: A Language for Data Analysis and Graphics. J. Comput. Graph. Stat. 5, pp. 299-314.

Jackson, W.E. (1997). Market Structure and the Speed of Price Adjustment: Evidence of NonMonotonicity, Review of Industrial Organization. 12, pp. 37-57.

Jeffreys, H. (1961). Theory of Probability ( $3^{\text {rd }}$ ed.). Oxford: Clarendon Press.

Judge, G., Griffiths, W., Hill, R., Lutkepohl, H. and Lee, T. (1985). The Theory and Practice of Econometrics. New York: John Wiley \& Sons.

Karrenbrock, J.D. (1991). The Behavior of Retail Gasoline Prices: Symmetric or Not? Federal Reserve Bank of St. Louis Review 73, pp. 19-29.

Kass, R.E. (1993). Bayes Factors in Practice. The Statistician 42, pp. 551-560.

Kass, R.E. and Raftery, A. (1995). Bayes Factors. Journal of the American Statistical Association 90, pp. 773-795.

Kass, R. E. and Wasserman, L. (1995). A Reference Bayesian Test for Nested Hypothesis and its Relationship to the Schwarz Criterion. Journal of the American Statistical Association 90, pp. 928-934.

Kinnucan, H.W. and Forker, O.D. (1987). Asymmetry in Farm-Retail Price Transmission for major Dairy Products. American Journal of Agricultural Economics 69, pp. 285-292.

Koop, G. and Potter, S. (1998). Bayes Factors and Nonlinearity: Evidence from Economic Time Series. Journal of Econometrics 88, pp. 251-281. 
Koop, G. and Potter, S. (1999). Dynamic Asymmetries in US Unemployment. Journal of Business and Economic Statistics 17, pp. 298-312.

Koop, G. (2003): Bayesian Econometrics. West Sussex, UK: Wiley.

Kovenock, D. and Widdows, K. (1998). Price Leadership and Asymmetric Price Rigidity. European Journal of Political Economy 14, pp. 167-187.

Kuran, T. (1983). Asymmetric Price Rigidity and Inflationary Bias. The American Economic Review 73(3), pp. 373-382.

Lancaster, T. (2004). An Introduction to Modern Bayesian Econometrics. Malden, MA: Blackwell.

Lee, P. M (1989). Bayesian Statistics: An Introduction. New York: Wiley and Sons.

LeSage, J. (1990). A Comparison of Forecasting Ability of ECM and VAR Models. The Review of Economics and Statistics 72, pp. 664- 671.

Levy, D., Bergen, M., Dutta, S. and Venable, R. (1997). The Magnitude of Menu Costs: Direct Evidence from Large U.S. Supermarket Chains. Quarterly Journal of Economics 112(3), pp. 791-825.

Lin, T. H., and Dayton, C. M. (1997). Model Selection Information Criteria for Non-nested Latent Class Models. J. Educat. Behav. Stat. 22, pp. 249-264.

Lloyd, T., McCorriston, S., Morgan, C. W. and Rayner, A. J. (2003). The Impact of Food Scares on Price Transmission in Inter-Related Markets. Paper presented to the XXVth IAAE Conference in Durban/South Africa.

Lopes and Salazar (2006) Bayesian Model Uncertainty in Smooth Transition Autoregressions. Journal of Time Series Analysis 27, pp. 99-117.

Lubrano, M. (1999). Bayesian Analysis of Nonlinear Time Series Models with a Threshold. In: Barnett et al, Nonlinear Econometric Modeling. Cambridge: Cambridge University Press.

Maindonald, J. and Braun, J. (2003). Data Analysis and Graphics using R. Cambridge, UK: Cambridge University Press.

Malcolm, R. F. (2000). Key Concepts in Model Selection: Performance and Generalizability. Journal of Mathematical Psychology 44, pp 205-231.

Manera, M. and Frey, G. (2007). Econometric Models of Asymmetric Price Transmission. Journal of Economic Surveys 67, pp. 349- 415.

Markon, K. E. and Krueger, R. F. (2004). An Empirical Comparison of InformationTheoretic Selection Criteria for Multivariate Behavior Genetic Models. Behavior Genetics. 34 (6), pp.593- 609. 
Marshall, A. (1936). Principles of Economics. London, Macmillan and Co., Appendix H, Section 3, pp. 807-809.

McCorriston, S., Morgan, C.W. and Rayner, A.J. (1998). Processing Technology, Market Power and Price Transmission. Journal of Agricultural Economics 49(2), pp. 185-201.

McCorriston, S., Morgan, C.W. and Rayner, A.J. (2001). Price Transmission: The Interaction between Market Power and Returns to Scale. European Review of Agricultural Economics 28(2), pp. 143-159.

McCorriston, S. (2002). Why Should Imperfect Competition Matter to Agricultural economists? European Review of Agricultural Economics 29(3), pp. 349-371.

McCulloch, R. E. and Rossi, P. (1992). Bayes Factors for Nonlinear Hypotheses and Likelihood Distributions. Biometrika 79, pp. 663-676.

Means, G. (1935). Industrial Prices and their Relative Inflexibility. Senate Discussion Paper No. 13, 74th U.S. Congress, 1 st session.

Meyer, J. (2003). Measuring Market Integration in the Presence of Transaction costs - A Threshold Vector Error Correction Approach. Contributed paper, XXV International Conference of Agricultural Economists, Durban, South Africa.

Meyer, J. and von Cramon-Taubadel, S. (2004): Asymmetric Price Transmission: A survey. Journal of Agricultural Economics 55 (3), pp. 581-611.

Miller, D.J. and Hayenga, M.L. (2001). Price Cycles and Asymmetric Price Transmission in the U.S. Pork Market. American Journal of Agricultural Economics 83, pp. 551-562.

Mohanty, S., Peterson, E.W.F. and Kruse, N.C. (1995). Price Asymmetry in the International Wheat Market. Canadian Journal of Agricultural Economics 43, pp. 355-366.

Myung Jae In (2000). The Importance of Complexity in Model Selection. Journal of Mathematical Psychology 44, pp. 190-204.

Neumark, D. and Sharpe, S.A. (1992). Market Structure and the Nature of Price Rigidity: Evidence from the Market for Consumer Deposits. Quarterly Journal of Economics 107, pp. 657-680.

Obstfeld, M. and Taylor, A. M. (1997). Nonlinear Aspects of Goods-Market Arbitrage and Adjustment; Heckscher's Commodity Points Revisited. Journal of the Japanese and International Economies 11, pp. 441-479.

Pauler, D. K. (1998). Schwarz Criterion and Related Methods for Normal Linear Models. Biometrika 85, pp.13-27.

Peltzman, S. (2000). Prices Rise Faster Than They Fall. Journal of Political Economy 108(3), pp. 466-502. 
Pfaff, B. (2006) Analysis of Integrated and Co-integrated Time Series with R. New York: Springer Science.

Pick D. H., Karrenbrock, J. D. and Carman, H. F. (1990). Price Asymmetry and Marketing Margin Behavior: an Example for California - Arizona Citrus. Agribusiness 6(1), pp. 75-84.

Poirier, D. (1995). Intermediate Statistics and Econometrics: A comparative Approach. Cambriage:The MIT Press.

Punyawadee, V., Boyd, M. S. and Faminow, M. D. (1991). Testing for Asymmetric Pricing in the Alberta Pork Market. Canadian Journal of Agricultural Economics 39, pp. 493-501.

Raftery, A.E. (1986). Choosing Models for Cross-Classifications. American Sociological Review 51, pp. 145-146.

Raftery, A. E. (1995). Bayesian Model Selection in Social Research, Sociological Methodology 25, pp. 111-163

Rafftery (1996). Approximate Bayes Factors and Accounting for Model Uncertainty in Generalized Linear models. Biometrika 83, pp. 251-66.

Reagan, P.B. and Weitzman, M.L. (1982). Asymmetries in Price and Quantity Adjustments by the Competitive Firm. Journal of Economic Theory 27, pp. 410- 420.

Romain, R., Doyon, M. and Frigon, M. (2002). Effects of State Regulations on Marketing Margins and Price Transmission Asymmetry: Evidence From the New York City and Upstate New York Fluid Milk Markets. Agribusiness 18(3), pp. 301-315.

Rossi, P. and Allenby, G. (2005). Bayesian Statistics and Marketing. West Sussex: John Wiley \& Sons Ltd.

Schertz Willett, L., Hansmire, M.R. and Bernand, J.C. (1997). Asymmetric Price Response Behavior of Red Delicious Apples. Agribusiness 13, No.6, pp. 649-658.

Scholnick, B. (1996). Asymmetric Adjustment of Commercial Bank Interest Rates: Evidence from Malaysia and Singapore. Journal of International Money and Finance 15, pp. 485-496.

Schroeder, T. C. (1988). Price Linkage between Wholesale and Retail Pork Cuts. Agribusiness 4(4), pp. 359-369.

Schwarz, G. (1978) Estimating the Dimension of a Model. Annals of Statistics 6, pp. 461464.

Sera, T. and Godwin, B. K. (2003). Price Transmission and Asymmetric Adjustment in the Spanish dairy sector. Applied Economics 35, pp. 1889-1999.

Shoesmith G.L. (1992). Cointegration, Error Correction and Improved Regional VAR Forecasting. Journal of Forecasting 11, pp. 91-109. 
Tierney, L. and Kadane, J.B. (1986). Accurate Approximations for Posterior Moments and Marginal Densities. Journal of the American Statistical Association 81, pp. 82-86.

Tong, H. (1983). Threshold Models in Non-Linear Time Series Analysis. New York, Springer Verlag.

Traill, B., Colman, D. and Young, T. (1978). Estimating Irreversible Supply Functions. American Journal of Agricultural Economics 60, pp. 528-531.

Tsay, R. S. (1989). Testing and Modeling Threshold Autoregressive Processes. Journal of the American Statistical Association 84, pp. 231-240.

Tweenten, L.G. and Quance, C.L. (1969). Positivistic Measures of Aggregate Supply Elasticities: Some new Approaches. American Journal of Agricultural Economics 51, pp. 342352.

V. Cramon-Taubadel, S. and Fahlbusch, S.(1994). Identifying Asymmetric Price Transmission with Error Correction models. Poster Session EAAE European Seminar in Reading.

V. Cramon-Taubadel, S. and Loy, J.-P. (1996). Price Asymmetry in the International Wheat Market: Comment. Canadian Journal of Agricultural Economics 44, pp. 311-317.

V. Cramon-Taubadel , S. (1996). An Investigation of Non-Linearity in Error Correction Representations of Agricultural Price Transmission. Contributed Paper, VIII Congress of the European Association of Agricultural Economists, Edinburgh.

V. Cramon-Taubadel ,S. (1998). Estimating Asymmetric Price Transmission with the Error Correction Representation: An Application to the German Pork Market", European Review of Agricultural Economics 25, pp. 1-18.

V. Cramon-Taubadel, S. and Loy, J.-P. (1999). The Identification of Asymmetric Price Transmission Processes with Integrated Time Series. Jahrbücher für Nationalökonomie und Statistik 218(1-2), pp. 85-106.

V. Cramon-Taubadel, S. and Meyer, J. (2000). Asymmetric Price Transmission: Fact or Artefact? University Göttingen, Institute for Agricultural Economics, Working Paper.

Vande Kamp, P.R. and Kaiser, H.M. (1999). Irreversibility in Advertising-Demand Response Functions: An Application to Milk. American Journal of Agricultural Economics 81, pp. 385396.

Ward, R.W. (1982). Asymmetry in Retail, Wholesale and Shipping Point Pricing for Fresh Vegetables. American Journal of Agricultural Economics 62, pp. 205-212.

Wasserman, L. (2000). Bayesian Model Selection and Model Averaging, Journal of Mathematical Psychology 44, pp. 92-107 
Wolffram, R. (1971). Positivistic Measures of Aggregate Supply Elasticities-Some New Approaches -Some Critical Notes. American Journal of Agricultural Economics 53, pp. 356356.

Wolffram, R. (2005). Estimation of Short- and Long -term Irreversible Relations, Institute of Agricultural Policy working paper, University of Bonn.

Yang, Y. (2003). Regression with Multiple Candidate Models: Selecting or Mixing? Statistica Sinica 13, pp783-809.

Young, T. (1980). Modeling Asymmetric Consumer Responses, with an Example. Journal of Agricultural Economics 31, pp. 175-186.

Yu, J., Meyer, R., and Berg, A. (2004). Deviance Information Criteria for Comparing Stochastic Volatility Models. Journal of Business and Economic Statistics 22 (1), pp. 107120.

Zellner, A. (1971). An Introduction to Bayesian Inference in Econometrics. New York: John Wiley and Sons.

Zellner, A. (1986b). On Assessing Prior Distributions and Bayesian Regression Analysis with g-Prior Distributions in P. K. Goel and Zellner, eds., Bayesian Inference and Decision Techniques: Essays in Honor of Bruno De Finetti. Armsterdam: North Holland.

Zhang, P., Fletcher, S and Carley, D (1995). Peanut Price Transmission Asymmetry in Peanut Butter. Agribusiness 11(1), pp.13-20.

Zucchini, W. (2000). An Introduction to Model Selection, Journal of Mathematical Psychology 44, pp. 41-61. 


\section{Appendix:}

\section{Appendix I}

\section{A Comparison of Alternative Approaches for Detecting Asymmetry: Standard ECM and Houck’s Model}

The findings of the survey by von Cramon-Taubadel and Meyer (2004) showed that the different methods detect asymmetry at different rates in a survey. Capps and Sherwell (2007) also conclude that differences in inferences are possible and conclusions derived from the von Cramon-Taubadel and Loy ECM approach in the Seattle and St Louis milk markets was not supported by the Conventional Houck's model in the same market with the same data.

Importantly, the F-test associated with the null hypothesis that retail prices respond symmetrically to increases and decreases in farm prices is not rejected in the case of Houck's model for above milk markets. In contrast, the hypothesis of symmetry is rejected in the ECM approach.

In agreement with these previous studies, this research uses simulated data to demonstrate that the different methods detect asymmetry at different rates. Interestingly, this holds when the true data generating process is symmetric.

$$
P_{A, t}=\beta_{o}+\beta_{1} P_{B, t}+\varepsilon_{t}
$$

$P_{A}$ and $P_{B}$ are either Non-stationary and cointegrated or Stationary and linearly dependent. The Conventional Houck model in first difference and the Standard Error Correction Model are denoted by HKD and ECM respectively.

Evidence derived from the Monte Carlo experiment using variants of simulated data are provided in Table 1 and indicates that alternative asymmetry test methods detect asymmetry at different rates. 
Table 1: Test for Asymmetry When the True Data Generating Process is Symmetric

\begin{tabular}{|c|c|c|}
\hline \multirow[t]{2}{*}{ Data Type } & \multicolumn{2}{|c|}{$\begin{array}{ll}\text { Symmetry } & \text { Rejected Percentages of Asymmetry } \\
\text { Test } & \\
& \text { (Based on 100 Replications) } \\
\end{array}$} \\
\hline & HKD & ECM \\
\hline Non Stationary and Cointegrated & 98 & 94 \\
\hline Stationary and Linearly Dependent & 98 & 96 \\
\hline \multirow[t]{2}{*}{ Data Type } & \multicolumn{2}{|c|}{$\begin{array}{l}\text { Symmetry Rejected Percentages of Asymmetry } \\
\text { Test } \\
\\
\text { (Based on } 1000 \text { Replications) }\end{array}$} \\
\hline & HKD & ECM \\
\hline Non Stationary and Cointegrated & 96.3 & 94.9 \\
\hline Stationary and Linearly Dependent & 96.5 & 94.7 \\
\hline
\end{tabular}

Appendix II

Table 2: Effects of Difference in Asymmetric Adjustment parameters (SECM)

\begin{tabular}{|l|l|l|l|}
\hline \multicolumn{5}{|c|}{ Difference in asymmetric adjustment parameters (0.25) } \\
\hline \multicolumn{5}{|c|}{ CECM } & HKD & SECM (DGP) \\
\hline Methods & $404(16.6 \%)$ & $421(1.4 \%)$ & $403(82 \%)$ \\
\hline AIC & $421(1.8 \%)$ & $432(2.9 \%)$ & $417(95.3 \%)$ \\
\hline BIC & $424(1.5 \%)$ & $434(3.7 \%)$ & $420 \quad(94.8 \%)$ \\
\hline DIC & $-213(3.5 \%)$ & $-218(3.3 \%)$ & $-211 \quad(93.2 \%)$ \\
\hline BMS & \multicolumn{5}{|c|}{ Model Fitted } \\
\hline Difference in asymmetric adjustment parameters (0.50) \\
\hline \multicolumn{5}{|c|}{ HKD } & SECM (DGP) \\
\hline CECM & $434(0 \%)$ & $401(83 \%)$ \\
\hline Methods & $402(17 \%)$ & $446(0.1 \%)$ & $416(96.5 \%)$ \\
\hline BIC & $420(3.4 \%)$ & $447(0.1 \%)$ & $418(96.9 \%)$ \\
\hline DIC & $423(3 \%)$ & $-225(0.1 \%)$ & $-211(95 \%)$ \\
\hline BMS & $-212(4.9 \%)$ & \\
\hline \multicolumn{5}{|c|}{$(12 \%)$} \\
\hline
\end{tabular}

Based on 1000 Replications 
Table 3: Effects of Difference in Asymmetric Adjustment Parameters (CECM)

\begin{tabular}{|l|l|l|l|}
\hline \multicolumn{5}{|c|}{ Difference in asymmetric adjustment parameters (0.25) } \\
\hline & CECM(DGP) & HKD & SECM \\
\hline Methods & & & \\
\hline AIC & $403(88.5 \%)$ & $422(0.5 \%)$ & $409(11 \%)$ \\
\hline BIC & $421(57.5 \%)$ & $440(7.8 \%)$ & $424(34.7 \%)$ \\
\hline DIC & $423(58.7 \%)$ & $443(9.3 \%)$ & $426(32 \%)$ \\
\hline BMS & $-213(69.3 \%)$ & $-222(6.8 \%)$ & $-215(23.9 \%)$ \\
\hline \multicolumn{5}{|c|}{ Model Fitted } \\
\hline Difference in asymmetric adjustment parameters (0.50) \\
\hline \multicolumn{5}{|c|}{ HKD } \\
\hline CECM (DGP) & SECM \\
\hline Methods & $402(88.9 \%)$ & $434(0 \%)$ & $408(11.1 \%)$ \\
\hline BIC & $420(65.7 \%)$ & $446(0.3 \%)$ & $423(34 \%)$ \\
\hline DIC & $423(67.6 \%)$ & $448(0.5 \%)$ & $425(31.9 \%)$ \\
\hline BMS & $-212(74.6 \%)$ & $-225(0.4 \%)$ & $-214(25 \%)$ \\
\hline \multicolumn{5}{|c|}{} \\
\hline
\end{tabular}

Based on 1000 Replications

Table 4: Concurrent Effects of Sample Size and Stochastic Variance (SECM)

\begin{tabular}{|c|c|c|c|}
\hline \multicolumn{4}{|c|}{ Large error size and small sample } \\
\hline \multicolumn{4}{|c|}{ Model Fitted } \\
\hline & CECM & HKD & SECM (DGP) \\
\hline \multicolumn{4}{|c|}{ Methods } \\
\hline AIC & $207(8 \%)$ & $206(53.9 \%)$ & $206(38.1 \%)$ \\
\hline $\mathrm{BIC}$ & $217(1.5 \%)$ & $213(71.8 \%)$ & $214(26.7 \%)$ \\
\hline DIC & $219(0.7 \%)$ & $215(76.2 \%)$ & $216(23.1 \%)$ \\
\hline BMS & $-115(0.1 \%)$ & $-112(80.7 \%)$ & $-113(19.2 \%)$ \\
\hline \multicolumn{4}{|c|}{ Small error size and Large sample } \\
\hline \multicolumn{4}{|c|}{ Model Fitted } \\
\hline & CECM & HKD & SECM (DGP) \\
\hline \multicolumn{4}{|c|}{ Methods } \\
\hline AIC & $208(15.3 \%)$ & $309(0 \%)$ & $207(84.7 \%)$ \\
\hline $\mathrm{BIC}$ & $226(2.6 \%)$ & $321(0 \%)$ & $222(97.4 \%)$ \\
\hline DIC & $228(2.2 \%)$ & $323(0 \%)$ & $224(97.8 \%)$ \\
\hline BMS & $-126(4.5 \%)$ & $-166(0 \%)$ & $-124(95.5 \%)$ \\
\hline
\end{tabular}

Based on 1000 replications 
Table 5: Concurrent Effects of Sample Size and Stochastic Variance (CECM)

\begin{tabular}{|l|l|l|l|}
\hline \multicolumn{4}{|c|}{ Large error size and small sample } \\
\hline \multicolumn{5}{|c|}{ Model Fitted } \\
\hline Methods & CECM(DGP) & HKD & SECM \\
\hline AIC & $206(11.4 \%)$ & $205(56.8 \%)$ & $206(31.8 \%)$ \\
\hline BIC & $216(3 \%)$ & $212(76.1 \%)$ & $214(20.9 \%)$ \\
\hline DIC & $219(2 \%)$ & $214(80.4 \%)$ & $217(17.6 \%)$ \\
\hline BMS & $-115(1 \%)$ & $-111(84.9 \%)$ & $-113(14.1 \%)$ \\
\hline \multicolumn{5}{|c|}{ Model Fitted } \\
\hline Small error size and large sample & HKD & SECM \\
\hline \multicolumn{5}{|l|}{ CECM(DGP) } & $210(0 \%)$ & $234(0 \%)$ \\
\hline Methods & $209(100 \%)$ & $322(0 \%)$ & $248(0.3 \%)$ \\
\hline AIC & $226(99.7 \%)$ & $324(0 \%)$ & $251(0.3 \%)$ \\
\hline BIC & $229(99.7 \%)$ & $-167(0 \%)$ & $-138(0 \%)$ \\
\hline DIC & $-125(100 \%)$ & \\
\hline BMS & & \\
\hline
\end{tabular}

Based on 1000 replications

Table 6: Concurrent Effects of Sample Size and Stochastic Variance

\begin{tabular}{|l|l|l|}
\hline & \multicolumn{1}{|c|}{ Recovery Rates (SWM) } \\
\hline $\begin{array}{l}\text { Selection } \\
\text { conditions }\end{array}$ & Unstable & Stable \\
\hline Methods & & \\
\hline AIC & $205(38.8 \%)$ & $206(94.5 \%)$ \\
\hline BIC & $212(46.6 \%)$ & $218(97.5 \%)$ \\
\hline DIC & $213(47.2 \%)$ & $220(97.5 \%)$ \\
\hline BMS & $-111(87 \%)$ & $-119(98 \%)$ \\
\hline & & \\
\hline
\end{tabular}

Based on 1000 replications

Table 7: Effects of Difference in Asymmetric Adjustment Parameters

\begin{tabular}{|l|l|l|}
\hline & \multicolumn{2}{|c|}{ Recovery Rates (SWM) } \\
\hline $\begin{array}{l}\text { Difference in Asymmetric } \\
\text { Adjustment Parameters }\end{array}$ & \multicolumn{1}{|c|}{0.25} & 0.50 \\
\hline Methods & & \\
\hline AIC & $401(46 \%)$ & $401(63.8 \%)$ \\
\hline BIC & $412(56.2 \%)$ & $413(78.3 \%)$ \\
\hline DIC & $413(56.6 \%)$ & $414(79.6 \%)$ \\
\hline BMS & $-207(89.6 \%)$ & $-208(96.5 \%)$ \\
\hline & & \\
\hline
\end{tabular}

Based on 1000 replications 
Appendix III

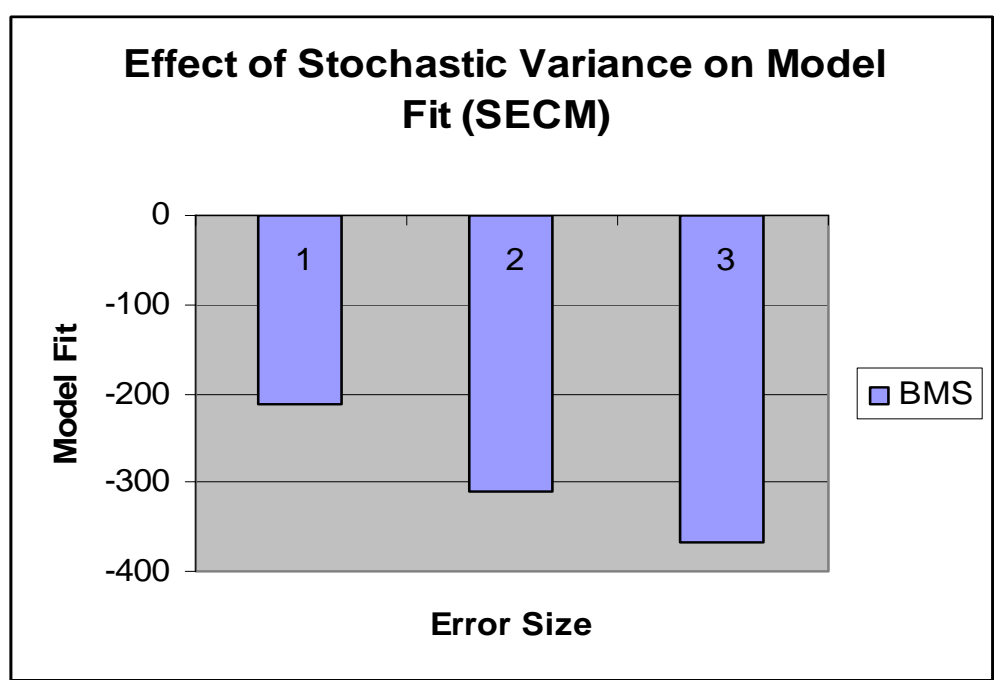

Figure 1: Example of Model fit as a function of Error Size (BMS)

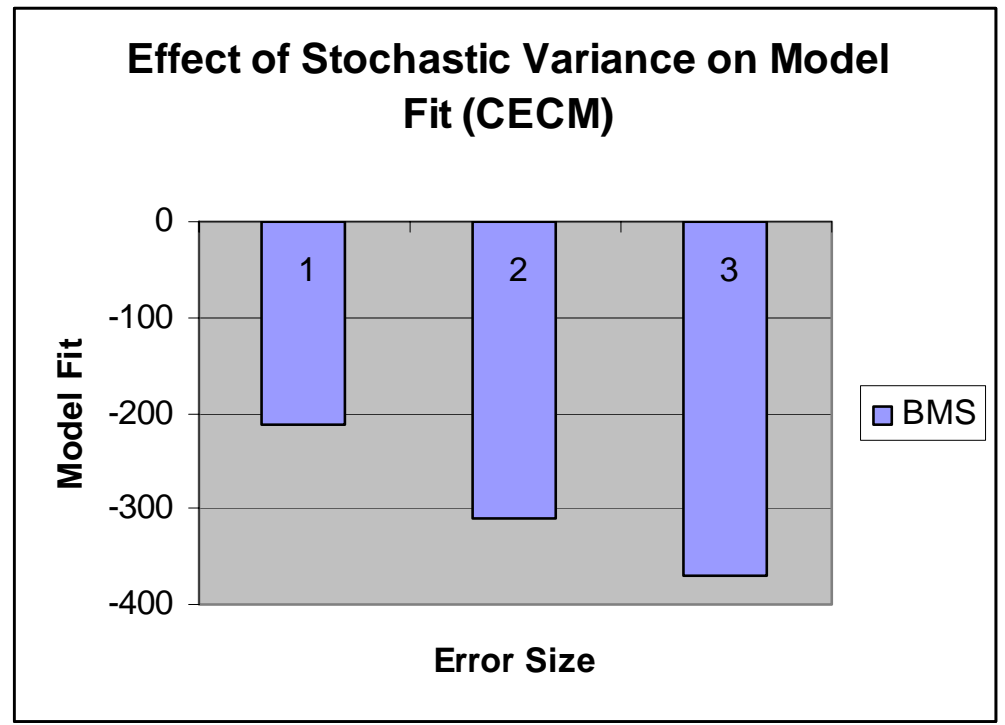

Figure 2: Example of Model fit as a function of Error Size (BMS) 


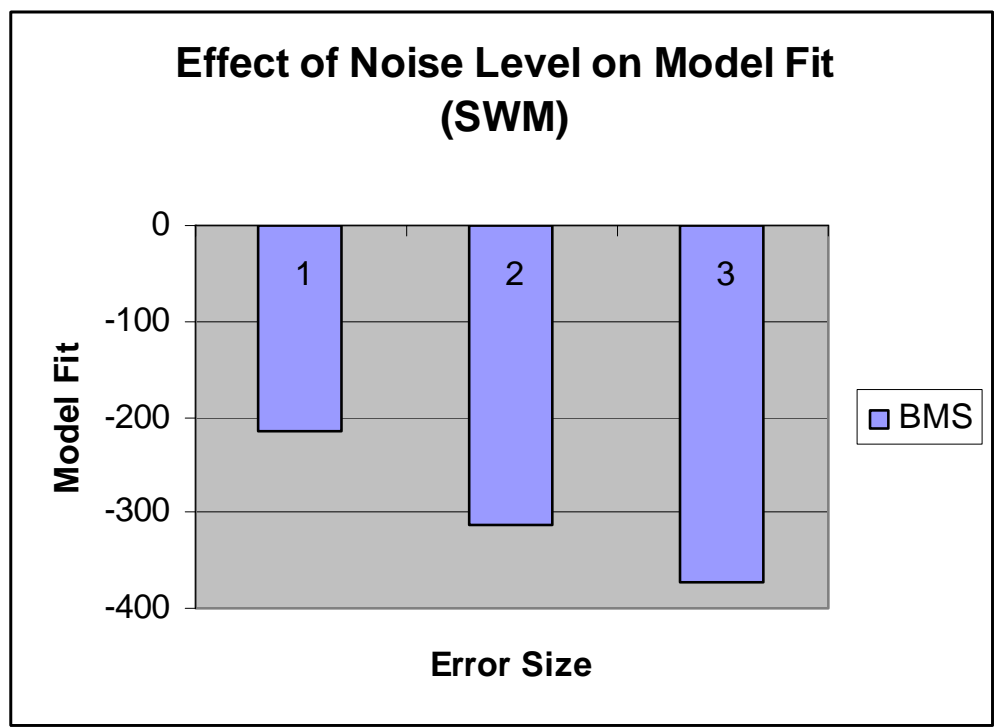

Figure 3: Example of Model fit as a function of Error Size (BMS)

\begin{abstract}
Appendix IV
$\mathrm{R}$ program codes of the data generating process (DGP) used in the analysis are provided on the compact disc attached to the back of the thesis. Computational algorithms for the Bayesian analysis can be down loaded at:
\end{abstract}

- Computational algorithms for the Bayesian analysis can be downloaded at :-

- http:www.wiley.co.uk./koopbayesian

- Site of Prof. Dr. Gary Koop

- Author of Bayesian Econometrics(2003)

- Alternatively it can also be downloaded from:

- http:www.biz.uiowa.edu./cbes.

- Site of Prof. Dr. John Geweke

- Author of Contemporary Bayesian Econometrics (2005)

- http://faculty.chicagogsb.edu/hedibert.lopes/research/

- Site of Prof. Dr. Hedibert Freitas Lopes

- Author of Stochastic Simulation for Bayesian Inference(2006) 


\section{Curriculum vitae}

\section{Personal data}

Name:

Henry De-Graft Acquah

Date of birth:

19.10.1970

Place of birth:

Takoradi, Ghana

Nationality:

Ghanaian

\section{Education}

Oct. 2004 - present:

Ph.D. Studies in Agricultural Economics, Department of Agricultural Economics and Rural Development, University of Goettingen, Germany. Concurrently with the Centre of Statistics University of Goettingen.

Feb.2006-June 2006: Attended an advanced training course: "Bayesian Methods in Theory and Practice" offered by Prof. Dr. John Geweke of the University of Iowa at the Wageningen University, Netherlands.

Training in "Modern Bayesian Econometrics" offered by Prof. Dr. Tony Lancaster of the Brown University at the University College London, UK.

Attended Seminar on Bayesian Inference in Econometrics and Statistics at the Unversity of Iowa, Iowa, U.S.A.

Training in Bayesian Data Analysis in the University of Göttingen.

Oct.2001-Oct.2003: $\quad$ M.Sc. Tropical and International Agriculture, Georg-August University, Göttingen Germany.

Oct.1993-Aug.1998: $\quad$ B.Sc. Agricultural Science, University of Cape Coast, Cape Coast Ghana

Oct.1993-Aug.1998: Diploma in Education, University of Cape Coast, Cape Coast Ghana.

\section{Employment Record}

June 1999-Oct 2001: Project Officer and Head of the Project Department, Nyarkrom Rural Bank limited, Ghana. 